\title{
A SINGLET EXTENSION OF THE MINIMAL SUPERSYMMETRIC STANDARD MODEL: TOWARDS A MORE NATURAL SOLUTION TO THE LITTLE HIERARCHY PROBLEM
}

\author{
A Dissertation \\ Submitted to the Graduate School \\ of the University of Notre Dame \\ in Partial Fulfillment of the Requirements \\ for the Degree of
}

Doctor of Philosophy

by

Alejandro de la Puente

Antonio Delgado, Director

Graduate Program in Physics

Notre Dame, Indiana

May 2012 
(c) Copyright by

Alejandro de la Puente

2012

All Rights Reserved 


\title{
A SINGLET EXTENSION OF THE MINIMAL SUPERSYMMETRIC STANDARD MODEL: TOWARDS A MORE NATURAL SOLUTION TO THE LITTLE HIERARCHY PROBLEM
}

\begin{abstract}
by

Alejandro de la Puente

In this work, I present a generalization of the Next-to-Minimal Supersymmetric Standard Model (NMSSM), with an explicit $\mu$-term and a supersymmetric mass for the singlet superfield, as a route to alleviating the little hierarchy problem of the Minimal Supersymmetric Standard Model (MSSM). I analyze two limiting cases of the model, characterized by the size of the supersymmetric mass for the singlet superfield. The small and large limits of this mass parameter are studied, and I find that I can generate masses for the lightest neutral Higgs boson up to $140 \mathrm{GeV}$ with top squarks below the TeV scale, all couplings perturbative up to the gauge unification scale, and with no need to fine tune parameters in the scalar potential.

This model, which I call the S-MSSM is also embedded in a gauge-mediated supersymmetry breaking scheme. I find that even with a minimal embedding of the S-MSSM into a gauge mediated scheme, the mass for the lightest Higgs boson can easily be above $114 \mathrm{GeV}$, while keeping the top squarks below the TeV scale.

Furthermore, I also study the forward-backward asymmetry in the $t \bar{t}$ system within the framework of the S-MSSM. For this purpose, non-renormalizable couplings between the first and third generation of quarks to scalars are introduced.
\end{abstract}


Alejandro de la Puente The two limiting cases of the S-MSSM, characterized by the size of the supersymmetric mass for the singlet superfield is analyzed, and I find that in the region of small singlet supersymmetric mass a large asymmetry can be obtained while being consistent with constraints arising from flavor physics, quark masses and top quark decays. 


\section{Dedication}

To Leven. I cannot describe with words how much you mean to me and how much I love you; I miss you every single day of my life. 


\section{CONTENTS}

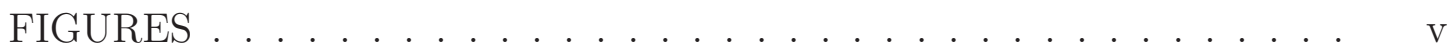

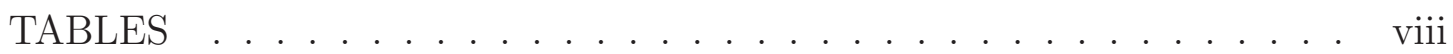

ACKNOWLEDGMENTS ................... ix

CHAPTER 1: INTRODUCTION . . . . . . . . . . . . . . . . 1

CHAPTER 2: SUPERSYMMETRY:

THE MINIMAL SUPERSYMMETRIC STANDARD MODEL . . . . . 7

2.1 Supersymmetry . . . . . . . . . . . . . . . . . . . 7

2.2 Supersymmetric Lagrangians: Algebraic Construction . . . . . . . 9

2.2.1 Dynamics of Chiral Supermultiplets . . . . . . . . . . 13

2.2 .2 Interactions and the Superpotential . . . . . . . . . . 16

2.2.3 Gauge Supermultiples and Gauge Interactions . . . . . . . 18

2.3 The Minimal Supersymmetric Standard Model . . . . . . . . . . . 23

2.3.1 The Superpotential . . . . . . . . . . . . . . . . 26

2.3.2 Soft Supersymmetry Breaking . . . . . . . . . . . . 28

2.3.3 The Higgs Potential and Electroweak Symmetry Breaking 30

2.3.4 Particle Spectrum . . . . . . . . . . . . . . . . . 34

2.3.5 One-loop effective potential . . . . . . . . . . . 37

CHAPTER 3: THE NEXT-TO-MINIMAL SUPERSYMMETRIC MODEL 42

3.1 Higgs potential and mass matrices . . . . . . . . . . . . . . 43

3.2 Limiting cases of the NMSSM . . . . . . . . . . . . . . . . 49

3.2 .1 The Effective MSSM . . . . . . . . . . . . . . . 49

3.2 .2 The Peccei-Quinn limit . . . . . . . . . . . . . 50

CHAPTER 4: A NATURAL SOLUTION TO THE LITTLE HIERARCHY PROBLEM:

THE S-MSSM . . . . . . . . . . . . . . . . . . . . 51

4.1 Model . . . . . . . . . . . . . . . . . . . . . . . . . . 51 
4.2 The large $\mu_{S}$ limit $\ldots \ldots \ldots \ldots \ldots \ldots \ldots \ldots \ldots$

4.2 .1 Spectrum . . . . . . . . . . . . . . . 55

4.2 .2 Perturbativity of $\lambda \ldots \ldots \ldots \ldots \ldots$

4.2 .3 Results for the large $\mu_{S}$ limit $\ldots \ldots \ldots$. . . . . . 62

4.3 The small $\mu_{S}$ limit $\ldots \ldots \ldots \ldots \ldots \ldots \ldots$

4.3 .1 Spectrum . . . . . . . . . . . . . . 67

4.3.2 Results for the small $\mu_{S}$ limit $\ldots \ldots \ldots \ldots$

4.4 Recent LHC results . . . . . . . . . . . . . . . . . 76

CHAPTER 5: GAUGE MEDIATED SUPERSYMMETRY BREAKING . 80

5.1 Fundamentals of GMSB . . . . . . . . . . . . . . . . 80

5.2 GMSB in the NMSSM . . . . . . . . . . . . . . . 83

5.3 GMSB in the S-MSSM . . . . . . . . . . . . . 87

CHAPTER 6: THE FORWARD-BACKWARD TOP ASYMMETRY . . . 96

6.1 Model . . . . . . . . . . . . . . . . . . . . . 97 97

6.2 Differential Cross Section and Asymmetry . . . . . . . . . . 100

6.3 Constraints . . . . . . . . . . . . . . . . . . . . . 103

$6.3 .1 u-t$ Mass Mixing . . . . . . . . . . . . . . 103

6.3 .2 Meson mixing . . . . . . . . . . . . . . . . . . . . 104

6.3.3 New Top decay channels . . . . . . . . . . . . . . . 105

6.3.4 Constraints from single and same-sign top production . . . 106

6.4 Results . . . . . . . . . . . . . . . . . . . . . 107

CHAPTER 7: CONCLUSIONS AND OUTLOOK . . . . . . . . . 114

APPENDIX A: . . . . . . . . . . . . . . . . . . 118

A.1 Gauge and Yukawa couplings . . . . . . . . . . . . 118

A.2 Gaugino Masses . . . . . . . . . . . . . . . . . . . 120

A.3 Trilinear couplings . . . . . . . . . . . . . . . . . 120

A.4 Squark, slepton and Higss soft masses . . . . . . . . . . 120

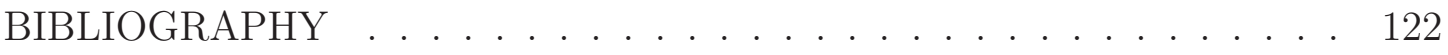




\section{FIGURES}

1.1 Quadratically divergent contributions to the Higgs mass parameter from fermions in the Standard Model. . . . . . . . . . . . . . . . 2

1.2 Quadratically divergent contributions to the Higgs mass parameter from (a) fermions and (b) their scalar superpartner. . . . . . . . . . . . 3

3.1 Mass of $h^{0}$ as a function of $A_{\lambda}$ for a typical NMSSM case with $\lambda=0.7$, $\kappa=0.05, M_{\tilde{g}}=500 \mathrm{GeV}$ and $m_{\tilde{t}}=1 \mathrm{TeV}$ in the maximal mixing scenario where $A_{t}=\sqrt{6} m_{\tilde{t}}$. . . . . . . . . . . . . .

4.1 The maximum value of $\lambda$ at the weak scale, consistent with perturbativity of all couplings up to the gauge unification scale, as a function of $\tan \beta . \ldots \ldots \ldots \ldots \ldots \ldots$

4.2 Lightest neutral Higgs mass as a function of $\tan \beta$ in the MSSM and S-MSSM. The red dashed/blue dotted curves were obtained using $\mu_{S}=$ $m_{\tilde{t}}=M_{\tilde{g}}=1 \mathrm{TeV}$ and $A_{\lambda}= \pm 1 \mathrm{TeV}$ in the S-MSSM. The solid black curve represents the MSSM. See the text for additional parameters used in the figure. . . . . . . . . . . . . . . . . . . . .

4.3 Lightest neutral Higgs mass as a function of $m_{\tilde{t}}$ in the MSSM (solid) and S-MSSM (dashed) for three values of $\tan \beta$ and assuming maximal mixing. See text for additional parameters used in the figure. . . . .

4.4 Scatter plot of $m_{h}$ (green) and $m_{h_{S}}$ (blue) as function of $m_{A}$ with a stop mass $m_{\tilde{t}}=500 \mathrm{GeV} A_{t}=0$. See text for additional parameters. . . .

4.5 Scatter plot of $m_{A_{S}}$ (green) as a function of $m_{A}$ with a stop mass $m_{\tilde{t}}=$ $500 \mathrm{GeV} A_{t}=0$, using the same parameter set as in Figure 4.4. . . .

4.6 Scatter plot of the ratio $\xi^{2}=\frac{g_{h_{S} Z Z}}{g_{h Z Z}^{S M}}$ as a fnuction of $m_{h_{S}}$ using the same parameter set as in Figure 4.4. . . . . . . . . . . . . . . .

4.7 Range of mass for $h$ for the S-MSSM and the MSSM as a function of $M_{S U S Y}$. See the text for additional parameters. . . . . . . .

4.8 Scatter plot for $m_{h^{0}}$ as a function of $\tan \beta$ fro the same parameters used in Figure $4.4 \ldots \ldots$. . . . . . . . . . . . . . 
4.9 The combined 95\% CL upper limits on the signal strength as a function of $m_{h^{0}}$ measured by the ATLAS detector; the solid curve indicates the observed limit and the dotted curve illustrates the median expected limit in the absence of a signal together with the $\pm 1 \sigma$ (green) and $\pm 2 \sigma$ (yellow) bands [35]. . . . . . . . . . . . . . . . . . . . . . . . 76

4.10 The combined 95\% CL upper limits on the signal strength as a function of $m_{h^{0}}$ measured by the CMS detector; the solid curve indicates the observed limit and the dotted curve illustrates the median expected limit in the absence of a signal together with the $\pm 1 \sigma$ (green) and $\pm 2 \sigma$ (yellow) bands [34]. . . . . . . . . . . . . . . . . .

5.1 Contributions to the MSSM gaugino masses in GMSB. . . . . . . . . 82

5.2 Contributions to the MSSM sfermion masses in GMSB. . . . . . . . . 83

5.3 Contributions to $m_{S}^{2}$ in a minimal setup of GMSB in the NMSSM. . . 84

5.4 Contributions to $m_{S}^{2}$ in a setup of GMSB where the singlet superfield of the NMSSM couples to the messenger sector. . . . . . . . . . . .

5.5 Contributions to $m_{S}^{2}$ in a setup of GMSB where the singlet superfield of the NMSSM couples to two sets of messenger fields. . . . . . . . .

5.6 Scatterplot of the lightest Higgs boson mass versus the effective stop mass, $M_{S U S Y}$, for $10^{4}$ randomly selected points with a messenger scale $M=10^{10} \mathrm{GeV}$ (black) and $10^{13} \mathrm{GeV}$ (red), and within the parameter space given in Equation (5.13). The two regions overlapalmost exactly, except at very larg $M_{S U S Y}$. The solid black line is the LEP bound of $114 \mathrm{GeV}$.

5.7 A scan of parameter space for $\tan \beta=2$ and $M=10^{13} \mathrm{GeV}$, varying $\mu$ and $\mu_{S} / \mu$ within the ranges specified in the text. All points in the figureare consistent with experimental bounds, inlcuding the bound on the Higgs mass of $114 \mathrm{GeV}$. The points are color coded by the light Higgs mass calculated from the model parameters, in $2 \mathrm{GeV}$ steps, beginning with $m_{h^{0}}<116 \mathrm{GeV}$ on the outside of the triangle and increasing to $m_{h^{0}}<118,120$ and $122 \mathrm{GeV}$ as one moves into the traingle and to the

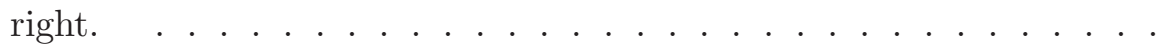

6.1 New diagrams contributing to $t \bar{t}$ production . . . . . . . . . . . .

6.2 New physics diagrams contributing to single top production together with a neutral Higgs in (a) and charged Higgs in (b). . . . . . . . . . 106 
6.3 The $t \bar{t}$ production cross section as a function of the parton level forwardbackward top asymmetry for various values of the singlet vev $v_{s}$, Scenarios A through D. The green band indicate the combined uncertainty from the asymmetry measurements of CDF and $\mathrm{D} \varnothing[2,3]$, and the cyan band the combined theoretical and experimental uncertainty on the value of the $t \bar{t}$ production cross section given in Equation (6.32) [110]. The value of $\Lambda_{31}$ increases along the curves, from 0 (left) to 9.5 (right) for $\Lambda_{13}$ close to zero. . . . . . . . . . . . . . . . . .

6.4 On the left plot the forward-backward top asymmetry at the parton level as a function of $\Lambda_{31}$ for scenarios A and B. The green bands indicate the combined uncertainty from the asymmetry measurements of CDF and $\mathrm{D} \varnothing[2,3]$. On the right, the $t \bar{t}$ production cross section as a function of $\Lambda_{31}$ for scenarios $\mathrm{A}$ and $\mathrm{B}$. The green bands indicate the combined theoretical and experimental uncertainty on the cross section [110].

6.5 On the left plot the forward-backward top asymmetry at the parton level as a function of $\Lambda_{31}$ for the large $\mu_{s}$ scenario. The orange line indicates a one $\sigma$ deviation from a combination of the independent CDF and $\mathrm{D} \varnothing$ asymmetry measurements $[2,3]$. On the right, the $t \bar{t}$ production cross section as a function of $\Lambda_{31}$. The green line corresponds to three $\sigma$ deviations away from the experimental cross section [110]. . . . . 


\section{TABLES}

2.1 THE MATTER CONTENT OF THE MSSM. . . . . . . . . . . . 25

5.1 A SAMPLING OF POINTS WITH HIGGS MASSESS ABOVE THE LEP BOUND. . . . . . . . . . . . . . . . . . . . . . . . . 95

6.1 SCALAR MIXING ANGLES AND VEV IN THE SINGLET FIELD DIRECTION. . . . . . . . . . . . . . . . . . . . 112 


\section{ACKNOWLEDGMENTS}

I would like to the thank my advisor Dr. Antonio Delgado for giving me the opportunity to work with him, and for his unconditional guidance throughout the course of this study as well as in my graduate career. I want to also thank Dr. Christopher Kolda for being a very pivotal piece in my advising team. Both Antonio and Chris, have provided me with the tools necessary to take the next step in what I believe will be a very successful career in physics. I want to also extend my thanks to the members of my dissertation committee for reviewing the thesis and providing their most valuable insight. Special thanks to Dr. Jorge de Blas Mateo, for his mentorship, understanding and friendship.

Special thanks to my friend and colleague Ayan Paul for his incredible unconditional friendship; especially through some very tough times in my life. I want to extend my gratitude to all the members of the Physics Department at the University for Notre Dame, for their guidence and support throughout the course of my studies.

I am very grateful to my partner and best friend Jennifer for being there with me throughout this journey; it was so incredibly better because your were with me. I would like to thank my mother, for her mentorship, dedication and hard work throughout my life, you are my superhero; my brother for being such an incredible friend and for believing so much in me. A special thanks to my dear friend Brian, even though we are a distance away, your wisdom an advise have 
been very influential in my life. Thanks to all of my family, you are truly my foundation. 


\section{CHAPTER 1}

\section{INTRODUCTION}

There are several arguments that may lead one to expect new physics extending the Standard Model to lie at the electroweak scale. One such argument is the cancellation of the quadratic divergences that generate the so-called "hierarchy problem" of the Standard Model. These divergences can be characterized by contributions to the mass parameter of the Standard Model Higgs field, $H$, arising from quantum corrections depicted in Figure 1.1 and expressed mathematically by:

$$
\delta m_{H}^{2}=-\frac{y_{f}^{2}}{8 \pi^{2}} \Lambda^{2}
$$

The parameter $\Lambda$ denotes an ultraviolet cut-off, which in the Standard Model is assumed to be $\gg M_{w e a k}$, and $y_{f}$ denotes the coupling strength between a massive fermion $f$ and the Higgs boson. In order for the mass parameter of the Standard Model Higgs boson to be on the order of $m_{H} \sim 100 \mathrm{GeV}$, one needs to appropriately adjust the bare mass parameter for the Higgs field which appears in the Standard Model Lagrangian. This tuning can be expressed in the following way:

$$
m_{H}^{2} \approx m_{H, b a r e}^{2}-\frac{y_{f}^{2}}{8 \pi^{2}} \Lambda^{2}
$$

In principle, the well founded principle of renormalization guarantees that all of the divergences are unobserved, yet it is believed that the Standard Model is only 


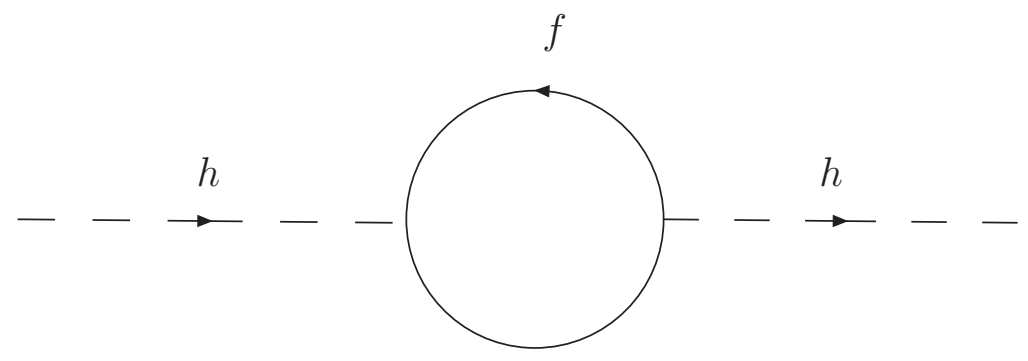

Figure 1.1. Quadratically divergent contributions to the Higgs mass parameter from fermions in the Standard Model.

a low energy description of nature and a more fundamental theory must exist at either the scale where the Standard Model forces unify, or ultimately the Planck scale, where the quantum nature of gravity must be taken properly into account. In either case, new heavier particles must exist beyond the scale $\Lambda$, which implies that their physical masses must now be tuned against an unphysical bare mass parameter in order to reproduce the mass of the Standard Model Higgs boson.

Supersymmetry (SUSY) avoids the order-by-order fine tuning that one imposes on the Higgs mass parameter of the Standard Model in favor of doubling the particle spectrum. The cancellation of the divergences is depicted in Figure 1.2 and can be expressed mathematically by:

$$
\delta m_{H}^{2}=\frac{\left(y_{\tilde{f}}-y_{f}^{2}\right)}{8 \pi^{2}} \Lambda^{2} .
$$

In the above equation, a superpartner for particle $f$, labeled by $\tilde{f}$ has been introduced. The SUSY framework dictates that $\tilde{f}$ carries a spin differing from $f$ by $1 / 2$ units. The superpartner, $\tilde{f}$, couples to the Higgs boson with strength $y_{\tilde{f}}$ equal to $y_{f}^{2}$, yielding a complete cancellation of the quadratic divergences.

But in supersymmetric theories, one also has $m_{f}=m_{\tilde{f}}$. Given the non- 


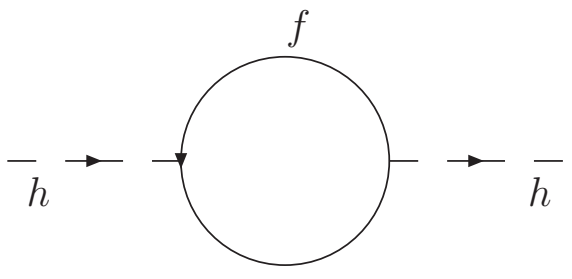

(a)

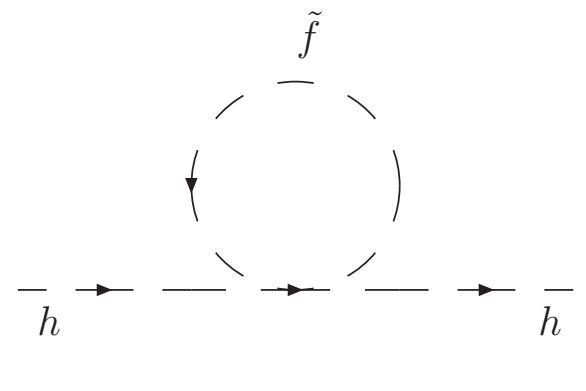

(b)

Figure 1.2. Quadratically divergent contributions to the Higgs mass parameter from (a) fermions and (b) their scalar superpartner.

observation of superpartners to date, it can be infered that SUSY must be broken; and in order not to reintroduce the quadratic divergences that vanish within SUSY theories, SUSY must be broken softly [72]. However, new contributions to the Higgs mass parameter arise from a softly broken SUSY and these are proportional to the mass splitting between a Standard Model particle and its superpartner. Provided that the scale of soft SUSY breaking is not too large, $m_{f} \gg m_{\tilde{f}}$, the resulting corrections to $m_{H}^{2}$ are small. Doubling of the spectrum is not without a cost, and several problems that are resolved naturally within the Standard Model, such as baryon and lepton number conservation and supression of flavor-changing neutral currents, require implementing additional symmetries. One such symmetry is $R$-parity $[49,66,106,112]$, which is implemented in order to avoid rapid proton decay within the minimal supersymmetric extension of the Standard Model known as the minimal SUSY Standard Model (MSSM).

For SUSY to be a viable solution to the hierarchy problem, the new supersymmetric partners must lie close to the weak scale. As the masses of the superpartners grow, fine-tunings which are proportional to the ratio between the weak scale mass, $m_{W}$, and the scale which parametrizes the superpartner masses, $m_{S U S Y}$, reappear. 
This problem may appear academic, given that none of the superpartner masses have been measured. But constraints from experiments have begun to force the MSSM into regions of parameter space where some tuning is required. The largest tuning comes from the experimental lower bound on the Standard Model Higgs boson of $114 \mathrm{GeV}$, obtained by the Large Electron Positron Collider (LEP) [12]. This constraint poses a problem for the MSSM for two reasons: First, in large regions of parameter space, the lightest scalar Higgs boson within the MSSM has the same properties as the Standard Model Higgs boson; therefore, the LEP lower bound of $114 \mathrm{GeV}$ is also applicable in the MSSM. Second, within the MSSM, the mass of the lightest Higgs boson is bounded from above at tree-level by the mass of the $\mathrm{Z}$ gauge boson, at $91 \mathrm{GeV}$. As a result, the mass of the lightest MSSM Higgs boson must be lifted above the LEP lower bound through large radiative corrections, if it is to avoid the LEP bound.

Within the MSSM, the radiative corrections to the lightest Higgs boson mass are logarithmically sensitive and in order for the lightest Higgs boson to evade the LEP bound, the mass of the supersymmetric partner of the top, the top squark, needs to be made quite heavy. These large top squark masses feed back into the mass parameters of the Higgs potential, destabilizing the weak scale hierarchy. Stabilizing the weak scale requires new tunings of the parameters in the Lagrangian. This smaller version of the original hierarchy problem, which I review in detail in the following chapter, is well known as the "little hierarchy problem" and is quite generic within the MSSM [13, 28, 33, 83].

In the last decade, a great deal of work has been done to uncover extensions to the MSSM which can naturally evade the LEP bound without the necessity of new tunings. These extensions include models where the SUSY Higgs evades 
experimental discovery and thus can lie below the LEP bound [44-46]; models in which the cut-off scale, $\Lambda$, is explicitly made to lie near the weak scale $[13,20,28$, $71,86,111]$ or models where the cut-off scale is lowered implicitly by imposing strong coupling on the theory $[30,38,68,80]$; models with new operators $[23$, $26,54]$ or symmetries $[11,16,17,19,20,88]$ incorporated into the MSSM; and models where new particles are added to the spectrum of the MSSM. This last class of models can be divided in two different types. In one, additional matter couples to the Higgs sector, and in such, generates new radiative corrections to the Higgs mass parameter $[11,16,17,19,20,32,47,70,88,92,93,105]$. The second avenue is to enlarge the Higgs sector with additional matter with the purpose of generating new quartic terms in the Higgs potential. These new quartic terms push up the mass of the Higgs at tree level, removing the need for large radiative corrections $[63,64,84]$.

In this thesis a minimal extension of the MSSM will be studied, where new matter fields are incorporated in the Higgs sector. I will study the contributions of new quartic terms to the mass of the Higgs field and I will motivate a natural solution to the little hierarchy problem. A subclass of these models has been studied in depth in the literature, but the motivation has been primarily to address the $\mu$-problem of the MSSM, defined later in this thesis. The minimal extension of the MSSM, called next-to-minimal SUSY Standard Model (NMSSM) (For reviews see $[62,89]$ ), incorporates one additional matter field not charged under the standard model gauge group, a gauge singlet, and thereby provides a natural solution to the $\mu$-problem. However, for the NMSSM to satisfactorily address both the little hierarchy problem and the $\mu$-problem, a certain amount of fine-tuning of the parameters of the model is crucial, as will be seen later in this work. The model 
studied in this thesis, which I label the singlet-extended MSSM (S-MSSM), is a variation on the minimal extension of the MSSM, in which the singlet is absolved of its responsibility for solving the $\mu$-problem. By doing so, it will be shown that a solution to the little hierarchy problem can be achieved in a more natural way.

In Chapter 2 of this thesis, I will present the MSSM, emphasizing primarily the Higgs sector and the scalar spectrum of the model. Within this chapter, I will also include all of the tools necessary for discussing the NMSSM and for the development of the S-MSSM. In Chapter 3, I will review the main aspects of the NMSSM and why it is a viable solution to the $\mu$-problem. I will also show the existence of fine-tuning in its parameters when asked to naturally address the little hierarchy problem in conjunction with the $\mu$-problem. In Chapter 4, I will introduce the S-MSSM and within its framework I will analyze two limiting cases characterized by the size of the singlet's mass [41, 42]. In Chapter 5, I explore whether the parameter choices that yield a more natural solution to the little hierarchy problem within the S-MSSM framework can be generated in a more complete model, in particular, a model where the SUSY-breaking masses are determined by only a few inputs. In order to do so, a very natural model of SUSY-breaking, namely gauge-mediated SUSY breaking (GMSB) is studied [40]. The relations of GMSB will be used to constrain the parameter space of the SMSSM. In Chapter 6, the Higgs spectrum is used to explain a recent experimental observation of the asymmetric production of $t \bar{t}$ pairs measured by the CDF [2] and DØ [3] collaborations. For this purpose, non-renormalizable couplings between the first and third generation of quarks to scalars are introduced [37]. Conclusions and outlook for future work will be discussed in Chapter 7 . 


\section{CHAPTER 2}

\section{SUPERSYMMETRY:}

\section{THE MINIMAL SUPERSYMMETRIC STANDARD MODEL}

In this chapter I will review general aspects of supersymmetric theories. A more complete and rigorous study of SUSY can be found in textbook treatments $[7,113]$. These can be supplemeneted with excellent and readily available reviews on the subject $[89,99]$. In this chapter I will introduce the properties of supersymmetric transformations and the types of superfields necessary to construct a minimal extension of the Standard Model. After introducing some relevant notation, I will walk the reader through the steps necessary to build a supersymmetric Lagrangian for both chiral and gauge supermultiplets. I will then introduce the Minimal Supersymmetric Standard Model, its matter content and properties such as $R$-parity as well soft SUSY-breaking interactions. A review of the Higgs potential of the MSSM and the requirements for electroweak symmetry breaking will follow. To conclude, I will review the spectrum of the MSSM and the radiative corrections that modify the Higgs mass at tree level.

\subsection{Supersymmetry}

In supersymmetric theories, a symmetry transformation can be used to turn a bosonic state into a fermionic state and vice-versa through the use of an anticommuting operator, $Q_{\alpha}$ and its hermitian conjugate $Q_{\dot{\alpha}}^{\dagger}$. (The subscript $\alpha$ denotes 
the spin index of the generators of SUSY.) Because these operators carry spin, it is clear that they must also be generators of spacetime symmetries. In fact, supersymmetry is the only symmetry which non-trivially combines the generators of the Poincare algebra of spacetime with those of an internal symmetry [75]. Specifically, if $P^{\mu}$ and $M^{\mu \nu}$ denote the generators of Lorentz transformations, then the generators $Q$ and $Q^{\dagger}$ must satisfy the following commutation and anti-commutation relations:

$$
\begin{aligned}
{\left[M^{\mu \nu}, Q_{\alpha}\right] } & =-i\left(\sigma^{\mu \nu}\right)_{\dot{\beta}}^{\alpha} Q^{\dagger \dot{\beta}} \\
\left\{Q_{\alpha}, Q_{\beta}\right\} & =\left\{Q_{\dot{\alpha}}^{\dagger}, Q_{\dot{\beta}}^{\dagger}\right\}=0 \\
\left\{Q_{\alpha}, Q_{\dot{\beta}}^{\dagger}\right\} & =2 \sigma_{\alpha \dot{\beta}}^{\mu} P_{\mu} .
\end{aligned}
$$

In SUSY, single-particle states from irreducible representations of the symmetry algebra, called supermultiplets. In general theories of SUSY, there exist an arbitrary number of generators, $Q_{\alpha}^{i}$, labeled by the index $i$. In this work I will only deal with $i=1$, known in the literature as $N=1$ supersymmetry. This is important since the Standard Model of particle physics is a chiral theory, and $N=1$ supersymmetry is the only supersymmetric framework that can naturally accommodate chiral fields. Each supermultiplet will contain both fermionic and bosonic degrees of freedom, and since the SUSY generators commute with the momentum squared operator, $P^{2}$, and also with the generators of gauge transformations, bosons and fermions within a supermultiplet will share the same mass and gauge quantum numbers. That is, within a supersymmetric extension of the Standard Model, fermions and bosons within a supermultiplet will have the same hypercharge, isospin and color quantum numbers.

In $N=1$ supersymmetry, the first possible supermultiplet contains a single 
Weyl spinor and two real scalars fields. Furthermore, both scalar degrees of freedom can be accommodated within one complex scalar field. This combination of a Weyl spinor with a complex scalar field is known as a chiral supermultiplet. The next possible supermultiplet consists of a massless spin one vector and a Weyl spinor, known as a gauge supermultiplet. In a minimal supersymmetric extension of the Standard Model, the known fundamental particles must be either part of a chiral or a gauge supermultiplet. Therefore, a fermion in the Standard Model will be part of a chiral supermultiplet which also contains a complex scalar superpartner, known as a sfermion. Gauge bosons will be part of a gauge supermultiplet which contains in addition a spin-1/2 superpartner (refered to as a gaugino.) Scalar fields, such as the Higgs, will form a chiral supermultiplet together with a spin-1/2 Weyl spinor known as a Higgsino.

Now that we have identified how physical states are accommodated within supermulitplets, we will review how supermultiplets transform, so that we may construct Lagrangians which are invariant under SUSY transformations.

\subsection{Supersymmetric Lagrangians: Algebraic Construction}

In order to build a supersymmetric Lagrangian within the $N=1$ supersymmetric framework, I will first review how to employ Weyl spinors following the formalism used in [7]. This formalism will prove more convenient than the usual language of Dirac and Majorana spinors. One can start by expressing a four component Dirac spinor in terms of two two-component Weyl spinors:

$$
\Psi_{D}=\left(\begin{array}{c}
\psi^{\dagger \dot{\alpha}} \\
\chi_{\alpha}
\end{array}\right)
$$


One can label $\psi$ as a right-handed chirality spinor using dotted spinor indices. The dagger to the left of the dotted spinor index is used only as a label which helps identify a spinor with a field that has a right handed chirality. The field $\psi$ has the following transformation property under the Lorentz gorup:

$$
\psi \rightarrow \psi^{\prime}=(1+i \epsilon \cdot \sigma / 2-\eta \cdot \sigma / 2) \psi \equiv V \psi .
$$

The parameters $\epsilon$ and $\eta$ denote rotation and boost parameters respectively, and $\sigma$ the set of Pauli matrices, which generate the Lorentz symmetry. The lower component $\chi$, with undotted spin indices, is used to denote a left-handed Weyl spinor and it transforms in the following way:

$$
\chi \rightarrow \chi^{\prime}=(1+i \epsilon \cdot \sigma / 2+\eta \cdot \sigma / 2) \chi \equiv V^{\dagger^{-1}} \chi .
$$

One very interesting feature of the above transformation properties can be uncovered by realizing that complex conjugation can be used to transform a left-handed spinor into a right-handed spinor. In other words, by complex conjugating Equation (2.4) and multiplying both sides of the equation by $\sigma_{2}$ one obtains:

$$
\begin{aligned}
\sigma_{2} \chi^{* \prime} & =\sigma_{2}\left(1-i \epsilon \cdot \sigma^{*} / 2+\eta \cdot \sigma^{*} / 2\right) \chi^{*} \\
& =\left(1-i \epsilon \cdot \sigma^{\dagger} / 2+\eta \cdot \sigma^{\dagger} / 2\right) \sigma_{2} \chi^{*} \equiv V \sigma_{2} \chi^{*} .
\end{aligned}
$$

Thus, the field $\sigma_{2} \chi^{*}$ has the same transformation properties as a right-handed spinor. Using Equations (2.5), one also concludes that $\sigma_{2}$ behaves as a raising and lowering operator of spinor indices. That is, the product of $\sigma_{2}$ with a left handed 
spinor,

$$
\sigma_{2}^{\dot{\alpha} \beta}\left(\chi_{\beta}\right)^{*}
$$

transforms as a right-handed spinor; likewise

$$
\sigma_{2 \alpha \dot{\beta}}\left(\psi^{\dagger \dot{\beta}}\right)^{*}
$$

transforms as a left-handed spinor. One can now use the properties introduced above to construct a Lorentz invariant combination which uses only left-handed Weyl spinors; (In a framework with only left-handed spinors, the dagger symbol will of course denote hermitian conjugation.) That is, given two left-handed Weyl spinors, $\xi$ and $\chi$, one can show that the following combination, $\left(i \sigma_{2} \xi^{*}\right)^{\dagger} \chi$ is Lorentz invariant:

$$
\left(i \sigma_{2} \xi^{*}\right)^{\dagger} \chi \rightarrow\left(V i \sigma_{2} \xi^{*}\right)^{\dagger} V^{\dagger^{-1}} \chi=\left(i \sigma_{2} \xi^{*}\right)^{\dagger} V^{\dagger} V^{\dagger^{-1}} \chi=\left(i \sigma_{2} \xi^{*}\right)^{\dagger} \chi
$$

A more convenient notation can be obtained by defining a left-handed dot product. Using this dot product, the Lorentz invariant combination introduced in the above equation can be written in the following way:

$$
\left(i \sigma_{2} \xi^{*}\right)^{\dagger} \chi=\left(i \sigma_{2} \xi\right)^{T} \chi \equiv \xi \cdot \chi
$$

In a similar way, one can check that $\xi^{\dagger} \chi^{\dagger}$ is also Lorentz invariant.

With these conventions in mind, one can now write a Dirac spinor in the 
following way:

$$
\Psi_{D}=\left(\begin{array}{l}
\xi^{c} \\
\chi
\end{array}\right)
$$

where $\xi^{c} \equiv i \sigma_{2} \xi^{*}$ denotes the charge conjugation of a two component left-handed Weyl spinor. The Dirac Lagrangian for $\xi$ and $\chi$ with Dirac mass $M$ is then given by:

$$
\mathcal{L}_{\text {Dirac }}=i \xi^{\dagger} \bar{\sigma}^{\mu} \partial_{\mu} \xi+i \chi^{\dagger} \sigma^{\mu} \partial_{\mu} \chi-M\left(\xi \cdot \chi+\xi^{\dagger} \cdot \chi^{\dagger}\right)
$$

where $\bar{\sigma}^{\mu}=\left(I_{2 \times 2},-\sigma\right)$. For Majorana particles of mass $M$, the four component spinor can be written as:

$$
\Psi_{M}=\left(\begin{array}{l}
\chi^{c} \\
\chi
\end{array}\right)
$$

with Lagrangian

$$
\mathcal{L}_{\text {Majorana }}=i \chi^{\dagger} \sigma^{\mu} \partial_{\mu} \chi-M\left(\chi \cdot \chi+\chi^{\dagger} \cdot \chi^{\dagger}\right)
$$

With the above conventions, one can now proceed with the task of constructing supersymmetric Lagrangians. In particular, I will begin by reviewing a simple supersymmetric model based on a free chiral supermultiplet. I will then summarize the transformation properties that leave this Lagrangian invariant. I will also explain how interactions among chiral supermultiplets can be introduced. Then I will introduce the Lagrangian for gauge supermultiplets and show how to implement gauge interactions within a supersymmetric framework. 


\subsubsection{Dynamics of Chiral Supermultiplets}

In this section I will review the steps needed to construct a Lagrangian for one free chiral supermultiplet and I will look at the conditions for its invariance under SUSY transformations. Recall that to guarantee the invariance of the action, $S$, under any transformation, it suffices that the Lagrangian density, $\mathcal{L}$, be invariant up to a total derivative. In a theory of one free chiral supermultiplet, consisting of a complex scalar field, $\phi$, and a left-handed Weyl spinor, $\chi$, the Lagrangian density can be expressed by [100]

$$
\mathcal{L}=\partial_{\mu} \phi^{\dagger} \partial^{\mu} \phi+\chi^{\dagger} i \bar{\sigma}^{\mu} \partial_{\mu} \chi .
$$

One would like to determine what the SUSY transformations are that link $\phi$ to $\chi$. In particular, SUSY transformations must take a complex scalar field into a lefthanded Weyl spinor. These type of transformations can be cast in the following way:

$$
\phi \rightarrow \phi^{\prime}=e^{i \theta \cdot Q} \phi,
$$

where $\theta$ are anticommuting numbers, Grassman variables independent of spacetime variables, and $Q_{\alpha}$ are the generators of SUSY introduced in Section 2.1. Under the SUSY transformation, $\phi$ changes by an infinitesimal amount $\theta_{\alpha}$ :

$$
\delta_{\theta} \phi \approx i \theta \cdot Q \phi
$$

One can use the commutation and anti-commutation relations of Equation (2.2) and rewrite the infinitesimal change in $\phi$ using the the Lorentz invariant product 
of two left-handed Wey spinors, that is [114]:

$$
\delta_{\theta} \phi \sim \theta^{\alpha} \chi_{\alpha}
$$

One can follow the same procedure to derive the transformation properties of a Weyl left-handed spinor. Along the lines of Equation (2.17), an infinitesimal change in $\chi$ with respect to the symmetry parameter, $\theta_{\alpha}$, can be expressed by:

$$
\delta_{\theta} \chi_{\alpha} \sim \theta_{\alpha} \phi
$$

To derive an exact expression one notes that in order for the above equation to be consistent, the right side must transform as a left-handed Weyl spinor. Also, since the field $\phi$ has mass dimension one and $\chi$ has mass dimension $3 / 2$, from Equation (2.17), the anticommuting parameter, $\theta$ must have a mass dimension of $-1 / 2$. Using these two facts and the Lorentz invariant combination $\sigma^{\mu} \partial_{\mu}$, Equation (2.18) becomes

$$
\delta_{\theta} \chi_{\alpha} \sim\left(\sigma^{\mu} \partial_{\mu} \phi\right) \theta_{\alpha}
$$

Unfortunately, the task is not complete. The Dirac equation for a Weyl spinor of mass $m, i \sigma^{\mu} \partial_{\mu} \chi=m \psi$, where $\psi$ is a right-handed Weyl spinor, dictates that the right hand side of Equation (2.19), as it is written, transforms as a right handed spinor. Hence, for both sides of the above equation to have the same Lorentz transformation properties, one must make use of Equation (2.5) to rewrite Equation (2.19) in the following way:

$$
\delta_{\theta} \chi=-i \sigma^{\mu}\left(i \sigma_{2} \theta^{*}\right) \partial_{\mu} \phi
$$


The negative sign follows by demanding that the Lagrangian in Equation (2.14) be invariant under the transformation for the fields $\phi$ and $\chi$ given by Equations (2.17) and (2.20) respectively. In fact, after some algebra [7], the action for a chiral supermultiplet is invariant if the Lagrangian in (2.14) changes by a total derivative:

$$
\delta_{\theta} \mathcal{L}=\partial_{\mu}\left(\chi^{\dagger} i \sigma_{2} \theta^{*} \partial^{\mu} \phi+\phi^{\dagger} \theta^{T}\left(-i \sigma_{2}\right) \partial^{\mu} \chi+\phi^{\dagger} \theta^{T} i \sigma_{2} \sigma^{\mu} \bar{\sigma}^{\nu} \partial_{\nu} \chi\right)
$$

The above equation was attained by demanding that the all particles be on shell, that is that $\phi$ and $\chi$ obey the Klein-Gordon and Dirac equations respectively. An analogous statement is that the SUSY algebra only closes on shell. For SUSY to be preserved by quantum correction one needs to equally account for off-shell degrees of freedom. To do this, one introduces an auxilliary field $F$ which has no kinetic term. The Lagrangian density for $F$ is given by:

$$
\mathcal{L}_{\text {aux }}=F^{*} F
$$

The field $F$ has mass dimension 2 and the following SUSY transformations [89]:

$$
\begin{aligned}
\delta_{\theta} F & =-i \theta^{\dagger} \bar{\sigma}^{\mu} \partial_{\mu} \chi, \\
\delta_{\theta} F^{*} & =i \partial_{\mu} \chi^{\dagger} \bar{\sigma}^{\mu} \theta .
\end{aligned}
$$

Under the above symmetry transformations, the Lagrangian in (2.22) transforms as

$$
\delta \mathcal{L}_{a u x}=\left(i \partial_{\mu} \chi^{\dagger} \bar{\sigma}^{\mu} \theta\right) F-F^{\dagger}\left(i \theta^{\dagger} \bar{\sigma}^{\mu} \partial_{\mu}\right)
$$

Again, the above contributions vanish when $\phi$ and $\chi$ are on shell, but for off-shell configurations one must have additional contributions to the SUSY transformation 
properties of the $\chi$ field. These are given by shifting $\delta_{\theta} \chi$ in Equation (2.20) such that:

$$
\delta_{\theta} \chi \rightarrow-i \sigma^{\mu}\left(i \sigma_{2} \theta^{*}\right) \partial_{\mu} \phi+\theta F
$$

This additional contribution to $\delta \chi$ provides $\delta_{\theta} \mathcal{L}$ with an additional term that cancels $\delta \mathcal{L}_{\text {aux }}$ up to a total derivative when the fields $\phi$ and $\chi$ are off-shell. One can now define a superfield $\Phi$ with components $(\phi, \psi, F)$ that transform under Equations (2.17), (2.25), and (2.23) and obtain a Lagrangian that is invariant under SUSY transformations for both on- and off-shell configurations. In fact one could have carried out the analysis of this section using the superfield framework. This is by far a more complete an rigorous analysis and I refer the reader to [113] for a detailed study.

\subsubsection{Interactions and the Superpotential}

In this section I will review how to generalize the Lagrangian for the chiral supermultiplet by introducing interactions between the fields. I will limit this review to the study of renormalizable interactions, that is, interaction terms with mass dimension 4. Under this condition, the most general interaction Lagrangian for a superfield $\Phi$ is given by:

$$
\mathcal{L}_{i n t}=W_{i}[\phi] F_{i}-\frac{1}{2} W_{i j}[\phi] \chi_{i} \cdot \chi_{j}+\text { h.c. }
$$

The functionals $W_{i}$ and $W_{i j}$ depend only on the scalar components of $\Phi$. Their structure can be determined by looking at the way they transform under Equations $(2.17),(2.23)$, and $(2.25)$. One can check that $W_{i j}$ must be symmetric in its indices $i$ and $j$ and that it must only depend on $\phi_{i}$ and not on its complex 
conjugate. The last property, will have very interesting consequences when trying to construct a model for a minimal SUSY extension of the Standard Model. The above two conditions allows one to write [7]:

$$
W_{i j}=M_{i j}+y_{i j k} \phi_{k} .
$$

$M_{i j}$ has dimensions of mass and is symmetric with respect to $i$ and $j$. The couplings $y_{i j k}$ are symmetric with respect to $i, j$ and $k$. One can express $W_{i j}$ in the following way:

$$
W_{i j}=\frac{\partial^{2} W}{\partial \phi_{i} \partial \phi_{j}},
$$

from which it follows that

$$
W=\frac{1}{2} M_{i j} \phi_{i} \phi_{j}+\frac{1}{6} y_{i j k} \phi_{i} \phi_{j} \phi_{k} .
$$

Similarly, one can arrive at the fact that $W_{i}$ must be related to $W$ in the following way:

$$
W_{i}=\frac{\partial W}{\partial \phi_{i}} .
$$

The above conditions guarantee the invariance of the interaction Lagrangian in Equation (2.26) under SUSY transformations up to a total derivative. $W$ contains all of the information regarding the interactions of the model and is commonly refered as the superpotential. From Equation (2.27), one can also see that the superpotential must be a holomorphic functional of the scalar components of the chiral supermultiplets $\Phi_{i}$. With all of these tools at hand, the full Lagrangian for one chiral supermultiplet can be expressed using the scalar and spinor kinetic 
terms and the superpotential, and it takes the following form:

$$
\mathcal{L}=\partial_{\mu} \phi^{\dagger} \partial^{\mu} \phi+\chi^{\dagger} i \bar{\sigma}^{\mu} \partial_{\mu} \chi+F^{\dagger} F+\left(\frac{\partial W}{\partial \phi} F-\frac{1}{2} \frac{\partial^{2} W}{\partial \phi^{2}} \chi \cdot \chi+h . c\right) .
$$

Because $F$ is a non-propagating field, one may wish to eliminate it from Equation (2.31) by using its equations of motion. That is, $\partial \mathcal{L} / \partial F=0$ which implies that $F^{\dagger}=-\partial W / \partial \phi$. Similarly for $F^{\dagger}$, where $F=-(\partial W / \partial \phi)^{\dagger}$. In this way, Equation (2.31) reduces to:

$$
\mathcal{L}=\partial_{\mu} \phi^{\dagger} \partial^{\mu} \phi+\chi^{\dagger} i \bar{\sigma}^{\mu} \partial_{\mu} \chi-\left|\frac{\partial W}{\partial \phi}\right|^{2}-\left(\frac{1}{2} \frac{\partial^{2} W}{\partial \phi^{2}} \chi \cdot \chi+h . c\right)
$$

The above equation can be generalized to an arbitrary number of chiral supermultiplets, $\Phi_{i}$ and becomes:

$$
\mathcal{L}=\partial_{\mu} \phi_{i}^{\dagger} \partial^{\mu} \phi_{i}+\chi_{i}^{\dagger} i \bar{\sigma}^{\mu} \partial_{\mu} \chi_{i}-\left|\frac{\partial W}{\partial \phi_{i}}\right|^{2}-\left(\frac{1}{2} \frac{\partial^{2} W}{\partial \phi_{i} \partial \phi_{j}} \chi_{i} \cdot \chi_{j}+h . c\right)
$$

\subsubsection{Gauge Supermultiples and Gauge Interactions}

In this section I will review the transformation properties for gauge supermultiplets in order to construct a SUSY-invariant Lagrangian for gauge interactions. I will begin by reviewing the construction for a massless $\mathrm{U}(1)$ abelian gauge theory and then generalize the findings to the non-abelian case.

One can derive a Lagrangian for a vector field $A$ and its superpartner, a spin 1/2 Weyl spinor, $\lambda_{\alpha}$, using a similar procedure to that used to derive the Lagrangian of a chiral supermultiplet. In fact, using our knowledge of electromagnetism and the Dirac equation, the Lagrangian for a massless gauge supermultiplet 
can be written in the following way:

$$
\mathcal{L}_{\text {gauge }}=-\frac{1}{4} F_{\mu \nu} F^{\mu \nu}+i \lambda^{\dagger} \bar{\sigma}^{\mu} \partial_{\mu} \lambda
$$

where $F^{\mu \nu}=\partial^{\mu} A^{\nu}-\partial^{\nu} A^{\mu}$. As with the chiral supermultiplet, one will want to derive the tranformation properties of $A$ and $\lambda$ such that Equation (2.34) is invariant under SUSY transformations. Furthermore, one will want this to hold for on- and off-shell degrees of freedom. The following SUSY transformations are consistent with both gauge and Lorentz invariance [7]:

$$
\delta_{\theta} A^{\mu}=\theta^{\dagger} \bar{\sigma}^{\mu} \lambda+\lambda^{\dagger} \bar{\sigma}^{\mu} \theta
$$

for a real vector field $A$ of mass dimension one and

$$
\delta_{\theta}=N \sigma^{\mu} \bar{\sigma}^{\nu} \theta F_{\mu \nu}
$$

for the spinor $\lambda$. The proportionality factor, $N$ will be determined by looking at how the Lagrangian transforms under the SUSY transformation. In particular, by looking at the variation of the gauge kinetic term, $\delta_{\theta}\left(-\frac{1}{4} F_{\mu \nu} F^{\mu \nu}\right)$ in $(2.34)$ one can check that $N=i / 2[7]$. However, the transformations in Equations (2.35) and (2.36) leave the Lagrangian in (2.34) invariant when both fields, $A$ and $\lambda$, are on shell. In order for the theory to be invariant when the fields are off-shell, a similar trick to that used in Section 2.2.1 is implemented. One introduces a non-propagating auxilliary field $K$ with the following Lagrangian:

$$
\mathcal{L}_{K}=\frac{1}{2} K^{2}
$$


The transformation properties for $K$ are derived in a very similar way as those for $F$, and in particular one finds that if:

$$
\delta_{\theta} K=-i\left(\theta^{\dagger} \bar{\sigma}^{\mu} \partial_{\mu}-\left(\partial_{\mu} \lambda\right)^{\dagger} \bar{\sigma}^{\mu} \theta\right),
$$

then the infinitesimal transformation law for $\lambda$, Equation (2.36), gets an additional contribution:

$$
\delta_{\theta} \lambda \rightarrow \frac{i}{2} \sigma^{\mu} \bar{\sigma}^{\nu} \theta F_{\mu \nu}+\theta K
$$

In this way, the Lagrangian that results from incorporating an auxilliary field $K$, given by

$$
\mathcal{L}_{\text {gauge }}=-\frac{1}{4} F_{\mu \nu} F^{\mu \nu}+i \lambda^{\dagger} \bar{\sigma}^{\mu} \partial_{\mu} \lambda+\frac{1}{2} K^{2},
$$

is invariant for both on- and off-shell configurations.

The U(1) example discussed above is not complete, it is an abelian case and if one wishes to build a minimal SUSY extension of the Standard Model, the nonabelian case must also be reviewed. In a non-abelian theory such as $\mathrm{SU}(\mathrm{N})$, the gauge fields belong to the adjoint representation of the group. In such a theory there exist $N^{2}-1$ gauge fields, $A^{a}$, and gauginos, $\lambda^{a}$ and these are labeled by a superscript, $a$ which runs from 1 to $N^{2}-1$. In order to generalize the Lagrangian in Equation (2.34) to the non-abelian case, one needs to note that the gaugino fields have a non-trivial gauge transformation given by [7, 100]:

$$
\lambda^{a} \rightarrow \lambda^{a \prime}=\lambda^{a}+g \epsilon^{a b c} \alpha^{b}(x) \lambda^{c} .
$$

Thus, to obtain a gauge invariant non-abelian theory, one must substitute the derivative term in Equation (2.34) with the covariant derivative, $D_{\mu}$, which in the 
adjoint representation is given by:

$$
D_{\mu}=\partial_{\mu}+i g T^{a} A_{\mu}^{a} .
$$

The Lagrangian for a non-abelian gauge supermultiplet is then given by:

$$
\mathcal{L}_{\text {gauge }}=-\frac{1}{4} F_{\mu \nu}^{a} F^{a, \mu \nu}+i \lambda^{a \dagger} \bar{\sigma}^{\mu} D_{\mu} \lambda^{a},
$$

where the matrices $T^{a}$ represent the generators of the group in the adjoint representation and $g$ can be identified with the gauge coupling constant.

As in the abelian case, one needs to incoporate an auxilliary field $K^{a}$ for each of the generators of the group in order to have a SUSY invariant gauge theory for both on- and off-shell configurations. The Lagrangian is an obvious generalization of the abelian case and it is given by:

$$
\mathcal{L}_{\text {gauge }}=-\frac{1}{4} F_{\mu \nu}^{a} F^{a, \mu \nu}+i \lambda^{a \dagger} \bar{\sigma}^{\mu} D_{\mu} \lambda^{a}+\frac{1}{2} K^{a} K^{a} .
$$

The SUSY transformations on the fields that leave the above Lagrangian invariant are summarized in $[7]$

$$
\begin{aligned}
\delta_{\theta} A^{a, \mu} & =\theta^{\dagger} \bar{\sigma}^{\mu} \lambda^{a}+\lambda^{a \dagger} \bar{\sigma}^{\mu} \theta \\
\delta_{\theta} \lambda^{a} & =\frac{1}{2} \bar{\sigma}^{\mu} \theta F_{\mu \nu}^{a}+\theta K^{a} \\
\delta_{\theta} K^{a} & =-i\left(\theta^{\dagger}\left(D_{\mu} \lambda\right)^{a}-\left(D_{\mu} \lambda\right)^{a \dagger} \bar{\sigma}^{\mu} \theta\right)
\end{aligned}
$$

Now that there is a framework to incorporate gauge fields and gauginos in a SUSY invariant manner, I will review how to introduce interactions between gauge and chiral supermultiplets. In an ordinary field theory, interactions between gauge 
fields with fermions and scalars is accomplished by substituting the derivative operator of the kinetic terms with the covariant derivative, taking into account the gauge transformation properties of both the scalar and the fermion. In a SUSY theory one can proceed in a similar way, and incorporate interactions between the vector field component of the gauge supermultiplet by modifying the kinetic terms in the chiral Lagrangian and then inspecting the SUSY invariance of the theory. In particular for a chiral supermultiplet, $\Phi$, one has the following modifications:

$$
\begin{gathered}
\partial_{\mu} \phi \rightarrow D_{\mu}=\partial_{\mu}+i g A_{\mu}^{a}\left(T^{a} \phi\right) \\
\partial_{\mu} \chi \rightarrow D_{\mu}=\partial_{\mu}+i g A_{\mu}^{a}\left(T^{a} \chi\right),
\end{gathered}
$$

where $g$ is the gauge coupling constant introduced in the previous section and the matrices $T^{a}$ are the generators of the gauge symmetry in the representation in which the fields $\phi$ and $\chi$ transform.

To incorporate interactions of the chiral supermultiplets with the gaugino and auxilliary components of the gauge supermultiplet, one can begin by inspecting any possible gauge and Lorentz invariant combinations of these fields. These are summarized in the following equation [7]:

$$
A g\left[\left(\phi^{\dagger} T^{a} \chi\right) \cdot \lambda^{a}+\lambda^{a \dagger} \cdot\left(\chi^{\dagger} T^{a} \phi\right)\right]+B g\left(\phi^{\dagger} T^{a} \phi\right) K^{a} .
$$

Both $A$ and $B$ will be determined by demanding that the above equation be invariant under SUSY transformations. In fact, using the transformation properties in Equations (2.17), (2.25), and (2.45), one can write the following SUSY invariant 
gauge-chiral Lagrangian for an arbitrary number of chiral supermultiplets:

$$
\begin{aligned}
\mathcal{L} & =\partial_{\mu} \phi_{i}^{\dagger} \partial^{\mu} \phi_{i}+\chi_{i}^{\dagger} i \bar{\sigma}^{\mu} \partial_{\mu} \chi_{i}-\left|\frac{\partial W}{\partial \phi_{i}}\right|^{2}-\left(\frac{1}{2} \frac{\partial^{2} W}{\partial \phi_{i} \partial \phi_{j}} \chi_{i} \cdot \chi_{j}+\text { h.c }\right) \\
& -\frac{1}{4} F_{\mu \nu}^{a} F^{a, \mu \nu}+i \lambda^{a \dagger} \bar{\sigma}^{\mu} D_{\mu} \lambda^{a}+\frac{1}{2} K^{a} K^{a} \\
& -\sqrt{2} g\left[\left(\phi^{\dagger} T^{a} \chi\right) \cdot \lambda^{a}+\lambda^{a \dagger} \cdot\left(\chi^{\dagger} T^{a} \phi\right)\right]-g\left(\phi^{\dagger} T^{a} \phi\right) K^{a} .
\end{aligned}
$$

As in the chiral case, one may choose to eliminate the auxilliary fields $K^{a}$ from the above Lagrangian. This task can be accomplished by using the equations of motion corresponding to $K^{a}$ given by

$$
K^{a}=g \phi_{i}^{\dagger} T^{a} \phi_{i} .
$$

\subsection{The Minimal Supersymmetric Standard Model}

In this section I will review the minimal supersymmetric extension of the Standard Model using the tools summarized in the previous section. As a first task, I will introduce the particle spectrum. To do this, it will prove useful to cast the fermionic mattter content of the Standard Model in terms of left-handed Weyl spinors. In particular, I will express all of the right-handed fermions in terms of charge conjugates of left-handed spinors, such that for any right handed fermion $f_{R}$,

$$
f_{R}=\left(\bar{f}_{L}\right)^{c}=i \sigma_{2}\left(\bar{f}_{L}\right)^{*} .
$$

The bar in the above equation is used to denote that $\bar{f}_{L}$ corresponds to the antiparticle of $f_{L}$ and as such, possesses opposite quantum numbers. As mentioned at the beginning of the previous section, each Standard Model fermion will belong to a chiral supermultiplet together with a complex scalar superpartner refered to 
as an sfermion. One then has for a left-handed chiral supermultiplet the following content:

$$
\hat{f}_{L}=\left(f_{L}, \tilde{f}_{L}\right) .
$$

The tilde is used to denote that the symbol $\tilde{f}$ is simply the scalar superpartner of $f$. Similarly for the antiparticles, one can define the following chiral supermultiplet:

$$
\hat{\overline{f_{L}}}=\left(\bar{f}_{L}, \tilde{\bar{f}}_{L}\right) .
$$

Gauge fields are grouped together with gauginos into gauge supermultiplets. There will be one supermultiplet for each of the generators of the Standard Model gauge group. They can be parametrized in the following way:

$$
\hat{A}^{a}=\left(A_{\mu}^{a}, \tilde{A}^{a}\right) .
$$

The tilde in this case is used to identify the fermion superpartner of the vector field $A_{\mu}^{a}$. In the Standard model one also incorporates a complex scalar field, $H$, in order to generate masses for up- and down- type fermions. In a supersymmetric theory, all of the information regarding the chiral supermultiplets is encoded in the superpotential and it was seen in Section 2.2.2 that the superpotential had to be a holomorphic functional of the scalar components of the chiral supermultiplets. Hence, in order to generate masses for the down-type quarks in a SUSY extension of the Standard Model one cannot just rely on $H^{\dagger}$, and one must incorporate an additional complex scalar field. In addition to properly generating masses for down-type quarks, the fermion superpartner of the new scalar field will also serve to cancel the anomalies that arise from extending $H$ to a superfield. A Higgs 
superfield can be parametrized as follows:

$$
\hat{H}=(H, \tilde{H})
$$

Again, the tilde in the above equation is reserved for the superpartner of a Standard Model Higgs, a Higgsino, which transforms as left-handed Weyl spinor.

TABLE 2.1

THE MATTER CONTENT OF THE MSSM.

\begin{tabular}{|c|c|c|c|}
\hline Superfield & Fermion & Scalar & $S U(3)_{c} \times S U(2)_{W} \times U(1)_{Y}$ \\
\hline \hline$\hat{Q}_{L}$ & $Q_{L}=\left(u_{L}, d_{L}\right)$ & $\tilde{Q}_{L}=\left(\tilde{u}_{L}, \tilde{d}_{L}\right)$ & $\mathbf{3} \times \mathbf{2} \times \mathbf{1} / \mathbf{6}$ \\
$\hat{\bar{u}}_{L}$ & $\bar{u}_{L}$ & $\tilde{\bar{u}}_{L}$ & $\overline{\mathbf{3}} \times \mathbf{1} \times \mathbf{- 2 / 3}$ \\
$\hat{\bar{d}}_{L}$ & $\bar{d}_{L}$ & $\tilde{\bar{d}}_{L}$ & $\overline{\mathbf{3}} \times \mathbf{1} \times \mathbf{1} / \mathbf{3}$ \\
\hline$\hat{E}_{L}$ & $E_{L}=\left(\nu_{L}, e_{L}\right)$ & $\tilde{E}_{L}=\left(\tilde{\nu}_{L}, \tilde{e}_{L}\right)$ & $\mathbf{1} \times \mathbf{2} \times \mathbf{- 1 / 2}$ \\
$\hat{\bar{e}}_{L}$ & $\bar{e}_{L}$ & $\tilde{\bar{e}}_{L}$ & $\mathbf{1} \times \mathbf{1} \times \mathbf{1}$ \\
\hline$\hat{H}_{u}$ & $\tilde{H}_{u}=\left(\tilde{H}_{u}^{+}, \tilde{H}_{u}^{0}\right)$ & $H_{u}=\left(H_{u}^{+}, H_{u}^{0}\right)$ & $\mathbf{1} \times \mathbf{2} \times \mathbf{1} / \mathbf{2}$ \\
$\hat{H}_{d}$ & $\tilde{H}_{d}=\left(\tilde{H}_{d}^{0}, \tilde{H}_{d}^{-}\right)$ & $H_{d}=\left(H_{d}^{0}, H_{d}^{-}\right)$ & $\mathbf{1} \times \mathbf{2} \times \mathbf{- 1 / 2}$ \\
\hline$\hat{G}$ & $\tilde{G}^{a}$ & $G_{\mu}^{a}$ & $\mathbf{8} \times \mathbf{1} \times \mathbf{0}$ \\
$\hat{A}$ & $\tilde{A}^{a}$ & $A_{\mu}^{a}$ & $\mathbf{1} \times \mathbf{3} \times \mathbf{0}$ \\
$\hat{B}$ & $\tilde{B}$ & $B_{\mu}$ & $\mathbf{1} \times \mathbf{1} \times \mathbf{0}$ \\
\hline
\end{tabular}

In Table 2.1, I summarize the spectrum of the MSSM together with their 
quantum number assignments under the Standard Model gauge group, $S U(3)_{c} \times$ $S U(2)_{W} \times U(1)_{Y}$. Notice that there are now two Higgs fields labeled by $H_{u}$ and $H_{d}$. In the following section, I will review the superpotential for the MSSM, some of its properties and look at the conditions for electroweak symmetry breaking as well as the effects that this has on the particle spectrum. In particular, I will review how different states mix after electroweak symmetry breaking.

\subsubsection{The Superpotential}

In this section I will review the form of the superpotential for the MSSM and discuss some of its properties and constrains that arise in order to build a phenomenological theory consistent with experimental observation. All of the ingredients necessary to incorporate chiral and gauge supermultiplets in a SUSY invariant way were introduced in Sections 2.2.1 - 2.2.3. In particular one can start to build a Lagrangian by recalling Equation (2.50):

$$
\begin{aligned}
\mathcal{L} & =\partial_{\mu} \phi_{i}^{\dagger} \partial^{\mu} \phi_{i}+\chi_{i}^{\dagger} i \bar{\sigma}^{\mu} \partial_{\mu} \chi_{i}-\left|\frac{\partial W}{\partial \phi_{i}}\right|^{2}-\left(\frac{1}{2} \frac{\partial^{2} W}{\partial \phi_{i} \partial \phi_{j}} \chi_{i} \cdot \chi_{j}+\text { h.c }\right) \\
& -\frac{1}{4} F_{\mu \nu}^{a} F^{a, \mu \nu}+i \lambda^{a \dagger} \bar{\sigma}^{\mu} D_{\mu} \lambda^{a}+\frac{1}{2} K^{a} K^{a} \\
& -\sqrt{2} g\left[\left(\phi^{\dagger} T^{a} \chi\right) \cdot \lambda^{a}+\lambda^{a \dagger} \cdot\left(\chi^{\dagger} T^{a} \phi\right)\right]-g\left(\phi^{\dagger} T^{a} \phi\right) K^{a}
\end{aligned}
$$

In order to derive all of the interactions present in the MSSM one will just need to specify a superpotential consistent with all of the symmetries of the Standard Model and which can reproduce all of the interactions currently tested by experimental collaborations. Because the Standard Model is a renormalizable theory, one may wish to start with only renormalizable interactions in the superpotential: That is one may begin by examining the following contributions to $W$ arising from 
the scalar components of the chiral supermultiplets:

$$
W=\mathbf{Y}_{\mathbf{u}} \tilde{\bar{u}}_{L} \tilde{Q}_{L} \cdot H_{u}-\mathbf{Y}_{\mathbf{d}} \tilde{\bar{d}}_{L} \tilde{Q}_{L} \cdot H_{d}-\mathbf{Y}_{\mathbf{e}} \tilde{\bar{e}}_{L} \tilde{L}_{L} \cdot H_{d}
$$

In the above equation I have suppressed the family index on the fermion supermutiplets and I have made use of "." to denote the $S U(2)_{W}$ antisymmetric invariant product, that is,

$$
Q \cdot H=Q_{1} H_{2}-Q_{2} H_{1}
$$

for two doublets of the $S U(2)$ Lie group. The Yukawa matrices $\mathbf{Y}_{\mathbf{u}}, \mathbf{Y}_{\mathbf{d}}$ and $\mathbf{Y}_{\mathbf{e}}$ are used to detemine the masses and Cabibbo-Kobayashi-Maskawa (CKM) mixing angles of the Standard Model quarks and leptons after electroweak symmetry breaking which occur after both $H_{u}$ and $H_{d}$ get a vacuum expectation value (vev). In Equation (2.57) one has also incorporated an additional term which couples $H_{u}$ to $H_{d}$, a $\mu$-term. This term is unique since it is the only holomorphic combination of $H_{u}$ and $H_{d}$ invariant under the symmetries of the Standard Model. Furthermore, in the sections to follow, I will show how this $\mu$-parameter stabilizes the electroweak hierarchy and provides mass terms for the gauginos and higgsinos.

In addition to the interactions introduced in Equation (2.57), there exist additional renormalizable operators that respect the gauge symmetries of the Standard Model but which violate total lepton number and baryon number. These interactions are summarized in [89]:

$$
\begin{aligned}
W_{\Delta L} & =\frac{1}{2} a^{i j k} \tilde{L}_{L i} \tilde{L}_{L j} \tilde{\bar{e}}_{L}+\frac{1}{2} b^{i j k} \tilde{L}_{L i} \tilde{Q}_{L j} \tilde{\bar{d}}_{L}+m^{i} \tilde{L}_{L i} \cdot H_{u} \\
W_{\Delta B} & =\frac{1}{2} c^{i j k} \tilde{\bar{u}}_{L i} \tilde{\bar{d}}_{L j} \tilde{\bar{d}}_{L k} .
\end{aligned}
$$

The above interactions violate baryon and lepton numbers by one unit. Lepton 
and baryon number violation in the MSSM are very troublesome, given that in the Standard Model, these are protected by accidental symmetries and such processes have not been observed experimentally. In order to have a viable SUSY extension of the Standard Model one is forced to introduce a symmetry that eliminates the interaction terms introduced in Equations (2.59) and (2.60). This symmetry, referred to as $R$ parity assigns each particle in the MSSM a conserved quantum number given by $[49,66,106,112]$ :

$$
P_{R}=(-1)^{3(B-L)+2 s},
$$

where $s$ is the spin of the particle, and $L$ and $B$ are lepton and baryon numbers respectively. From the above equation, one can deduce that particles within the same supermultiplet do not have the same $R$-parity and using this conventions one notes that all of the Standard Model particles have $R=1$ parity and their superpartners have $R=-1$ parity. Besides forbidding lepton and baryon violation, $R$-parity has interesting phenomenological consequences. Most importantly, it states that the lightest superpartner must be completely stable, thus providing an excellent candidate for dark matter. In the following chapter, I will briefly mention how stable dark matter candidates arise in singlet extensions of the MSSM.

\subsubsection{Soft Supersymmetry Breaking}

Now that I have reviewed what goes into building the superpotential of the MSSM one must account for the non-observation of superpartners. As far as we have been able to test, there is no clear evidence that superpartners are lighter than the energies we are currently able to probe. Recall, from Section 2.1 that the momentum squared operator, $P^{2}$, commutes with the generators of SUSY, $Q_{\alpha}$. 
One then is able to conclude that in a SUSY theory both bosonic and fermionic degrees of freedom must have the same mass. Clearly SUSY must be broken. The mechanism of SUSY breaking is beyond the scope of this thesis, however, it is believed that SUSY must be broken spontaneously, where the vacuum state of the theory is not invariant under SUSY transformations, but the dynamics are. Several mechanism have been proposed such as the Fayet-Iliopoulos supersymmetry breaking $[67,101]$ and O'Raifeartaigh supersymmetry breaking [87]. In this work, I will review the concept of soft SUSY breaking, that is, the parametrization of SUSY breaking interactions through the addition of terms in the MSSM lagrangian which explicitly break SUSY. As discussed in Chapter 1, these additional terms do not reintroduce the quadratic divergences on the Higgs mass parameter that were eliminated by adopting a SUSY framework in the first place [72]. Thus by incorporating soft SUSY breaking interactions one is able to cling to SUSY as a solution to the hierarchy problem.

In order to incorporate all possible soft SUSY breaking interactions, one must keep in mind that they must remain invariant under the Standard Model gauge symmetry as well as R-parity. For the MSSM the soft SUSY breaking interactions are summarized in [89]:

$$
\begin{aligned}
\mathcal{L}_{\text {soft }} & =-\frac{1}{2}\left(M_{3} \tilde{g}^{a} \tilde{g}^{a}+M_{2} \tilde{W}^{a} \tilde{W}^{a}+M_{1} \tilde{B} \tilde{B}+h . c\right) \\
& -\left(\mathbf{a}_{\mathbf{u}} \tilde{\bar{u}}_{L} \tilde{Q}_{L} \cdot H_{u}-\mathbf{a}_{\mathbf{d}} \tilde{\bar{d}}_{L} \tilde{Q}_{L} \cdot H_{d}-\mathbf{a}_{\mathbf{e}} \tilde{\bar{e}}_{L} \tilde{L}_{L} \cdot H_{d}+h . c\right) \\
& -\mathbf{M}_{\mathbf{Q}}^{2} \tilde{Q}_{L}^{\dagger} \tilde{Q}_{L}-\mathbf{M}_{\mathbf{L}}^{2} \tilde{L}_{L}^{\dagger} \tilde{L}_{L}-\mathbf{M}_{\overline{\mathbf{u}}}^{2} \tilde{\bar{u}}_{L}^{\dagger} \tilde{\bar{u}}_{L}-\mathbf{M}_{\overline{\mathbf{d}}}^{2} \tilde{\bar{d}}_{L}^{\dagger} \tilde{\bar{d}}_{L}-\mathbf{M}_{\overline{\mathbf{e}}}^{2} \tilde{\bar{e}}_{L}^{\dagger} \tilde{\bar{e}}_{L} \\
& -m_{H_{u}}^{2} H_{u}^{\dagger} H_{u}-m_{H_{d}}^{2} H_{d}^{\dagger} H_{d}-\left(B_{\mu} H_{u} \cdot H_{d}+h . c\right) .
\end{aligned}
$$

The parameters $M_{3}, M_{2}$ and $M_{1}$ denote soft masses for the gaugino fields. The 
matrices, $\mathbf{a}_{\mathbf{u}}, \mathbf{a}_{\mathbf{d}}$ and $\mathbf{a}_{\mathbf{e}}$ correspond to trilinear soft terms which lead to mixing between $\tilde{u}_{L}$ with $\tilde{\bar{u}}_{L}$ after electroweak symmetry breaking, and $\mathbf{M}_{\mathbf{Q}}^{2}, \mathbf{M}_{\overline{\mathbf{u}}}^{2}, \mathbf{M}_{\overline{\mathbf{d}}}^{2}$, $\mathbf{M}_{\mathbf{L}}^{2}$, and $\mathbf{M}_{\overline{\mathbf{e}}}^{2}$ correspond to squark and slepton soft SUSY breaking mass terms. These matrices will be approximately flavor diagonal, otherwise they will introduce dangerous contributions to flavor changing neutral currents and additional sources of $\mathrm{CP}$ violation beyond those that have been observed. In fact, in order to guarantee that the only CP violating phase that arises is from the CKM matrix all soft parameters must have exactly the same phase. Furthermore, the trilinear soft terms must also be proportional to the Yukawa couplings given in the MSSM superpotential, that is, one can write $\mathbf{a}_{\mathbf{u}} \rightarrow \mathbf{A}_{\mathbf{u}} \mathbf{Y}_{\mathbf{u}}$, where for up-type quarks $\mathbf{A}_{\mathbf{u}}$ is diagonal with entries $A_{u}, A_{c}$, and $A_{t}$. In addition, one also includes soft SUSY breaking mass terms for $H_{u}$ and $H_{d}$ given my $m_{H_{u}}^{2}, m_{H_{d}}^{2}$ as well as bilinear term parametrized by $B_{\mu}$.

The MSSM Lagrangian is now complete and one can proceed to study the Higgs potential and the conditions for electroweak symmetry breaking. This analysis will be used to derive the masses for up- and down-type fermions as well as the rich new phenomenology consisting of new supersymmetric particles.

\subsubsection{The Higgs Potential and Electroweak Symmetry Breaking}

In order to study electroweak symmetry breaking in the MSSM one must first derive the Higgs potential from Equations (2.50) and (2.62). That is, one must find quadratic and quartic terms for the fields $H_{u}$ and $H_{d}$. The first set of quadratic terms can be obtained by differentiating the superpotential of the MSSM in (2.57) with respect to $H_{u}$ and $H_{u}$ following the analysis in Section 2.2.2. One then is 
able to obtain the $F$-term contributions to the Higgs potental:

$$
\begin{aligned}
V_{F} & =|\mu|^{2} H_{u}^{\dagger} H_{u}+|\mu|^{2} H_{d}^{\dagger} H_{d} \\
& =|\mu|^{2}\left(\left|H_{u}^{+}\right|^{2}+\left|H_{u}^{0}\right|^{2}\right)+|\mu|^{2}\left(\left|H_{d}^{-}\right|^{2}+\left|H_{d}^{0}\right|^{2}\right) .
\end{aligned}
$$

The $K$-term contributions to the Higgs potential are derived using the equations of motion for the auxilliary fields $K^{a}$ given in Equation (2.50). They yield the following quartic terms in the Higgs potential:

$$
V_{K}=\frac{g^{2}+g^{\prime 2}}{8}\left(\left|H_{u}^{+}\right|^{2}+\left|H_{u}^{0}\right|^{2}-\left|H_{d}^{-}\right|^{2}-\left|H_{d}^{0}\right|^{2}\right)^{2}+\frac{g^{2}}{2}\left|H_{u}^{+} H_{d}^{0 \dagger}+H_{u}^{0} H_{d}^{-\dagger}\right|^{2},
$$

where $g^{\prime}$ and $g$ are the gauge couplings associated with the gauge groups $U(1)_{Y}$ and $S U(2)_{W}$ respectively. The above two contributions to the Higgs potential together with the soft SUSY breaking contributions to the MSSM Lagrangian in (2.62), yield the following Higgs potential:

$$
\begin{aligned}
V_{H} & =\left(|\mu|^{2}+m_{H_{u}}^{2}\right)\left(\left|H_{u}^{+}\right|^{2}+\left|H_{u}^{0}\right|^{2}\right)+\left(|\mu|^{2}+m_{H_{d}}^{2}\right)\left(\left|H_{d}^{-}\right|^{2}+\left|H_{d}^{0}\right|^{2}\right) \\
& +\left\{B_{\mu}\left(H_{u}^{+} H_{d}^{-}-H_{u}^{0} H_{d}^{0}\right)+h . c\right\} \\
& +\frac{g^{2}+g^{\prime 2}}{8}\left(\left|H_{u}^{+}\right|^{2}+\left|H_{u}^{0}\right|^{2}-\left|H_{d}^{-}\right|^{2}-\left|H_{d}^{0}\right|^{2}\right)^{2}+\frac{g^{2}}{2}\left|H_{u}^{+} H_{d}^{0 \dagger}+H_{u}^{0} H_{d}^{-\dagger}\right|^{2}
\end{aligned}
$$

One is now ready to determine under which conditions the above potential has a minimum which breaks the $S U(2)_{W} \times U(1)_{Y}$ symmetry of the Standard Model down to Electromagnetism. Because of the $S U(2)_{W}$ invariance of the Higgs potential, it is possible to rotate one of the components of $H_{u}$ away such that at the 
minimum of the potential:

$$
\begin{aligned}
H_{u}^{+} & =0 \\
\frac{\partial V}{\partial H_{u}^{+}} & =0 .
\end{aligned}
$$

The above two conditions automatically yield $H_{d}^{-}=0$. Therefore, neither $H_{u}^{+}$and $H_{d}^{-}$acquire vevs and in this way the electromagnetic invariance of the vacuum state is not spoiled. Setting the charged components to zero and minimizing the potential with respect to the neutral components, $H_{u}^{0}$ and $H_{d}^{0}$, one arrives at the following condition which must be satisfied in order to obtain a non-zero minimum:

$$
\left(|\mu|^{2}+m_{H_{u}}^{2}\right)\left(|\mu|^{2}+m_{H_{d}}^{2}\right) \leq\left|B_{\mu}\right|^{2}
$$

Additionally, inspecting the potential along the direction $H_{u}^{0}= \pm H_{d}^{0}$ one also arrives at an additional condition that must be met in order to obtain a non-zero vev:

$$
m_{H_{u}}^{2}+m_{H_{d}}^{2}+2|\mu|^{2} \geq 2 B_{\mu}
$$

Thus, at the minimum one has:

$$
\begin{gathered}
\left\langle H_{u}^{0}\right\rangle=v_{u} \\
\left\langle H_{d}^{0}\right\rangle=v_{d},
\end{gathered}
$$


Miinimizing the Higgs potential and incorporating the above constraints yields the following two minimization conditions:

$$
\begin{aligned}
\sin 2 \beta & =\frac{2 B_{\mu}}{m_{H_{u}}+m_{H_{d}}+2 \mu^{2}+\lambda^{2} v^{2}} \\
\frac{1}{2} m_{Z}^{2} & =\frac{m_{H_{d}}^{2}-m_{H_{u}}^{2} \tan ^{2} 2 \beta}{\tan ^{2} \beta-1}-\mu^{2},
\end{aligned}
$$

where $m_{Z}=\left(g^{2}+g^{2}\right) v^{2} / 2$ and $v^{2}=v_{u}^{2}+v_{d}^{2}$ together with $\tan \beta=v_{u} / v_{d}$. One very important property of the MSSM is that it must reproduce the mass of the $Z$ gauge boson implying that $v=174 \mathrm{GeV}$.

I will like to point out some relevant issues associated with the analysis of the Higgs potential. It is important to emphasize that in the Higgs potential all parameters can be chosen to be real. This is possible since one is free to rescale the fields by a global phase. In this way, it is straightforward to check that any phase associated with $\mu$ and $B_{\mu}$ can be absorbed into the redefinitions of the fields. It is possible that CP violation in the MSSM may occur at the one loop level. This has been studied in the literature and has very interesting phenomenological consequences $[10,102]$. One may also wonder what effects do the other scalars in the MSSM have on electroweak symmetry breaking, in particular, the appearance of minima along the sfermion directions which may turn out to be deeper than the electroweak symmetry breaking minima. Minima in these directions would break the color and charge symmetry of the Standard Model and this will be problematic given that there has been no experimental evidence of such a phenomenom. However, it has been shown that the invariance of the Higgs potential under color and charge symmetries can constrain the parameter space of the MSSM. The analysis is beyond the scope of this thesis and I will refer the 
reader to detailed studies provided by the following references [8, 73].

\subsubsection{Particle Spectrum}

In this section, I will review the spectrum of the MSSM after electroweak symmetry breaking. I will begin by reviewing the Higgs spectrum. The Higgs sector contains eight degrees of freedom, three which are eaten and provide the masses for the Standard Model gauge bososn, $Z$ and $W^{ \pm}$. The remaining five degrees of freedom correspond to one CP-odd state, $A^{0}$, two CP-even states, $h^{0}$ and $H^{0}$, and two charged scalars, $H^{ \pm}$. The masses can be derived from the second order term in an expansion of the Higgs potential around the electroweak symmetry breaking minimum, $v_{u}$ and $v_{d}$. They are given by:

$$
\begin{aligned}
m_{A^{0}}^{2} & =\frac{2 B_{\mu}}{\sin 2 \beta} \\
m_{H^{ \pm}}^{2} & =m_{A^{0}}^{2}+m_{W}^{2} \\
m_{h^{0}}^{2} & =\frac{1}{2}\left\{m_{A^{0}}^{2}+m_{Z}^{2}-\sqrt{\left(m_{A^{0}}^{2}+m_{Z}^{2}\right)^{2}-4 m_{A^{0}}^{2} m_{Z}^{2} \cos ^{2} \beta}\right\} \\
m_{H^{0}}^{2} & =\frac{1}{2}\left\{m_{A^{0}}^{2}+m_{Z}^{2}+\sqrt{\left(m_{A^{0}}^{2}+m_{Z}^{2}\right)^{2}-4 m_{A^{0}}^{2} m_{Z}^{2} \cos ^{2} \beta}\right\}
\end{aligned}
$$

One important property of the MSSM is that the only scalar mass which is not unconstrained, is the mass of $h^{0}$. This mass is bounded from above by:

$$
m_{h^{0}}^{2} \leq m_{Z}^{2} \cos ^{2} \beta
$$

In Chapter 1, I mentioned that the experimental lower bound on the mass of the lightest Higgs boson of the MSSM was the same experimental bound that applies to the Standard Model Higgs. Because this bound is set at $114 \mathrm{GeV}$ [12], the upper bound given in Equation (2.78) has already been experimentally exclueded. 
Fortunately, it is known that the Higgs boson receives additional contributions to its mass from radiative corrections. The main contributions to the Higgs mass arise from the top quark and its scalar superpartner. In the next section I will review the form of this contributions using the one-loop effective potential approach. I will also review the fine-tuning problem that arises in the MSSM by incorporating large radiative corrections to raise the mass of the light Higgs above the experimental lower bound.

After electroweak symmetry breaking, the neutral higgsinos, $\left(\tilde{H}_{u}^{0}, \tilde{H}_{d}^{0}\right)$, and neutral gauginos, $\left(\tilde{B}, \tilde{A}^{3}\right)$, transform in the same way under the electromagnetic gauge symmetry. These fields will mix and yield four mass eigenstates referred to as neutralinos. These masses and mixings can be derived from Equations (2.28), together with the $K$-terms and soft SUSY breaking terms in the MSSM Lagrangian. These are parametrized by the following matrix:

$$
N=\left(\begin{array}{cccc}
M_{1} & 0 & -m_{W} \sqrt{\frac{\alpha_{1}}{\alpha_{2}}} \cos \beta & m_{W} \sqrt{\frac{\alpha_{1}}{\alpha_{2}}} \sin \beta \\
0 & M_{2} & m_{W} \cos \beta & -m_{W} \sin \beta \\
-m_{W} \sqrt{\frac{\alpha_{1}}{\alpha_{2}}} \cos & m_{W} \cos \beta & 0 & \mu \\
m_{W} \sqrt{\frac{\alpha_{1}}{\alpha_{2}}} \sin \beta & -m_{W} \sin \beta & \mu & 0
\end{array}\right)
$$

where $\alpha_{1} / \alpha_{2}=g^{\prime 2} / g^{2}$. Diagonalizing $N$ yields the neutralino mass eigenstates.

Similarly, the charged higgsinos, $\left(\tilde{H}_{u}^{+}, \tilde{H}_{d}^{-}\right)$, and charged gauginos, $\left(\tilde{A}^{1}, \tilde{A}^{2}\right)$, mix and yield two mass eigenstates referred to as charginos. The masses and mixings are derived as in the neutral case, and are parametrized by:

$$
C=\left(\begin{array}{cc}
M_{2} & \sqrt{2} m_{W} \sin 2 \beta \\
\sqrt{2} m_{W} \cos 2 \beta & \mu
\end{array}\right)
$$


Standard Model fermions acquire mass through their Yukawa couplings in the MSSM superpotential. These can be derived using Equation (2.28). After electroweak symmetry breaking the mass terms for standard model fermions are given by:

$$
\begin{aligned}
& m_{u}^{i}=y_{u}^{i} v \sin \beta \\
& m_{d}^{i}=y_{d}^{i} v \cos \beta \\
& m_{e}^{i}=y_{e}^{i} v \cos \beta .
\end{aligned}
$$

The superscript $i$ is used to denote the family index, and $y_{u}^{i}, y_{d}^{i}$ and $y_{e}^{i}$ are the diagonal components of the Yukawa matrices introduced in Equation (2.57).

The masses for the scalar superpartners of the Standard Model fermions can be derived by combining contributions from the superpotential, $F$-terms, together with the soft SUSY breaking terms given in Equation (2.62). Because the trilinear mass terms are proportional to the Yukawa matrices, $\mathbf{a} \approx \mathbf{A Y}$, these are essentially zero for the first two generations of quarks and leptons and one does need to consider mixing between the $\tilde{f}_{L}$ and $\tilde{f}_{L}$ : The masses for the first two generations are summarized below [89]:

$$
\begin{aligned}
m_{\tilde{e}_{L}, \tilde{\mu}_{L}}^{2} & =m_{L_{1}, L_{2}}^{2}+m_{e, \mu}^{2}-m_{Z}^{2}\left(\frac{1}{2}-\sin ^{2} \theta_{W}\right) \cos 2 \beta \\
m_{\tilde{\tilde{e}}_{L}, \tilde{\mu}_{L}}^{2} & =m_{\bar{e}, \bar{\mu}}^{2}+m_{e, \mu}^{2}-m_{Z}^{2} \sin ^{2} \theta_{W} \cos 2 \beta \\
m_{\tilde{\nu}_{e}, \tilde{\nu}_{\mu_{L}}}^{2} & =m_{L}^{2}+\frac{1}{2} m_{Z}^{2} \cos 2 \beta \\
m_{\tilde{u}_{L}, \tilde{c}_{L}}^{2} & =m_{Q_{1}, Q_{2}}^{2}+m_{u, c}^{2}+m_{Z}^{2}\left(\frac{1}{2}-\frac{2}{3} \sin ^{2} \theta_{W}\right) \cos 2 \beta
\end{aligned}
$$




$$
\begin{aligned}
& m_{\tilde{\tilde{u}}_{L}, \tilde{c}_{L}}^{2}=m_{\bar{u}, \bar{c}}^{2}+m_{u, c}^{2}+\frac{2}{3} m_{Z}^{2} \sin ^{2} \theta_{W} \cos 2 \beta \\
& m_{\tilde{d}_{L}, \tilde{s}_{L}}^{2}=m_{Q_{1}, Q_{2}}^{2}+m_{d, s}^{2}-\left(\frac{1}{2}-\frac{1}{3} m_{Z}^{2} \sin ^{2} \theta_{W}\right) \cos 2 \beta \\
& m_{\tilde{d}_{L}, \tilde{s}_{L}}^{2}=m_{\bar{d}, \bar{s}}^{2}+m_{d, s}^{2}-\frac{1}{3} m_{Z}^{2} \sin ^{2} \theta_{W} \cos 2 \beta,
\end{aligned}
$$

where $\sin \theta_{W}=g^{\prime} / \sqrt{g^{\prime 2}+g^{2}}$. For the third generation, the mixing is not longer small, and $\tilde{f}_{L}$ will mix with $\tilde{\bar{f}}_{L}$ for $f=t, b, \tau$. The physical states can be obtained by diagonalizing the following matrices:

$m_{\tilde{t}_{1}, \tilde{t}_{2}}^{2}=\left(\begin{array}{cc}m_{Q_{3}}^{2}+m_{t}^{2}+m_{Z}^{2}\left(\frac{1}{2}-\frac{2}{3} \sin ^{2} \theta_{W}\right) \cos 2 \beta & m_{t}\left(A_{t}+\mu / \tan \beta\right) \\ m_{t}\left(A_{t}+\mu / \tan \beta\right) & m_{\bar{u}}^{2}+m_{t}^{2}+\frac{2}{3} m_{Z}^{2} \sin ^{2} \theta_{W} \cos 2 \beta\end{array}\right)$,

$m_{\tilde{b}_{1}, \tilde{b}_{2}}^{2}=\left(\begin{array}{cc}m_{Q_{3}}^{2}+m_{b}^{2}-m_{Z}^{2}\left(\frac{1}{2}-\frac{1}{3} \sin ^{2} \theta_{W}\right) \cos 2 \beta & m_{b}\left(A_{b}+\mu \tan \beta\right) \\ m_{b}\left(A_{b}+\mu \tan \beta\right) & m_{\bar{b}}^{2}+m_{b}^{2}-\frac{1}{3} m_{Z}^{2} \sin ^{2} \theta_{W} \cos 2 \beta\end{array}\right)$,

$m_{\tilde{\tau}_{1}, \tilde{\tau}_{2}}^{2}=\left(\begin{array}{cc}m_{L_{3}}^{2}+m_{\tau}^{2}-m_{Z}^{2}\left(\frac{1}{2}-\sin ^{2} \theta_{W}\right) \cos 2 \beta & m_{\tau}\left(A_{\tau}+\mu \tan \beta\right) \\ m_{\tau}\left(A_{\tau}+\mu \tan \beta\right) & m_{\bar{\tau}}^{2}+m_{\tau}^{2}-m_{Z}^{2} \sin ^{2} \theta_{W} \cos 2 \beta\end{array}\right)$.

\subsubsection{One-loop effective potential}

In this section I will review the one-loop effective potential formalism to derive the one loop radiative corrections to the Higgs masses. The dominant top and top squark mass contributions have been widely studied $[22,56,57,76]$, and in this section, I will only focus on the mass of the lightest Higgs in order to review the origin of a fine tuning problem within the MSSM.

The one-loop effective potential of the MSSM consists of two pieces. The First 
one was introduced in Section 2.3.3 and is basically the tree-level Higgs potential:

$$
\begin{aligned}
V_{H}^{0}(Q) & =\left(|\mu|^{2}+m_{H_{u}}^{2}\right)\left(\left|H_{u}^{+}\right|^{2}+\left|H_{u}^{0}\right|^{2}\right)+\left(|\mu|^{2}+m_{H_{d}}^{2}\right)\left(\left|H_{d}^{-}\right|^{2}+\left|H_{d}^{0}\right|^{2}\right) \\
& +\left\{B_{\mu}\left(H_{u}^{+} H_{d}^{-}-H_{u}^{0} H_{d}^{0}\right)+h . c\right\} \\
& +\frac{g^{2}+g^{\prime 2}}{8}\left(\left|H_{u}^{+}\right|^{2}+\left|H_{u}^{0}\right|^{2}-\left|H_{d}^{-}\right|^{2}-\left|H_{d}^{0}\right|^{2}\right)^{2}+\frac{g^{2}}{2}\left|H_{u}^{+} H_{d}^{0 \dagger}+H_{u}^{0} H_{d}^{-\dagger}\right|^{2} .
\end{aligned}
$$

The parameters in the tree-level Higgs potential are to be evaluated at the scale $Q$. This can be accomplished by using the renormalization group equations (RGEs) of the MSSM given in Appendix A. The second contribution to the Higgs potential is given by the one loop corrections to the self-energies of the Higgs fields. This contribution can be summarized by:

$$
V_{H}^{1-\text { loop }}(Q)=\frac{1}{64 \pi^{2}} S \operatorname{Tr}\left[\mathcal{M}^{4}\left(\log \frac{\mathcal{M}^{2}}{Q^{2}}-\frac{3}{2}\right)\right],
$$

where $\mathcal{M}^{2}$ is used to denote the field dependent mass matrices for the MSSM spectrum. They can be obtained by retaining the field dependence in all of the masses introduced in the previous section, that is, the masses one will obtain before electroweak symmetry breaking. The symbol $S T r$ is used to denote the supersymmetric trace, since now one has to sum over both fermion and boson degrees of freedom. A complete derivation of the one loop effective potential can be found in the following reference [104]. As in the tree-level potential, all parameters appearing in the one-loop contribution are evaluated at an energy scale, $Q$.

Using the one-loop effective potential one can proceed to finding the conditions for electroweak symmetry breaking. With this approach one will see that one-loop corrections to the values $v_{u}$ and $v_{d}$ will arise. One can then proceed to calculate the 
Higgs spectrum by deriving the second order terms in an expansion of the Higgs potential around the one-loop corrected minimum. In the analysis that follows I will only review the leading correction to the lightest Higgs of the MSSM. A useful expression for the Higgs radiative corrections is given in the Higgs decoupling limit. In this limit, the mass of the CP-odd Higgs, $m_{A^{0}} \rightarrow \infty$, and the tree-level mass of the lightest Higgs, $h^{0}$ takes it maximal value of $m_{h^{0}}^{2} \approx m_{Z}^{2} \cos ^{2} \beta$. Furthermore, in this limit the one-loop radiative corrections are given by [54]:

$$
\begin{aligned}
\delta m_{h^{0}}^{2} & \approx \frac{12}{16 \pi^{2}} \frac{m_{t}^{4}}{v^{2}}\left[\log \left(\frac{m_{\tilde{t}_{1}} m_{\tilde{t}_{2}}}{m_{t}^{2}}\right)+\frac{X_{t}^{2}}{m_{\tilde{t}_{1}}^{2}-m_{\tilde{t}_{2}}^{2}} \log \left(\frac{m_{\tilde{t}_{1}}^{2}}{m_{\tilde{t}_{2}}^{2}}\right)\right. \\
& \left.+\frac{1}{2}\left(\frac{X_{t}^{2}}{m_{\tilde{t}_{1}}^{2}-m_{\tilde{t}_{2}}^{2}}\right)^{2}\left(2-\frac{m_{\tilde{t}_{1}}^{2}+m_{\tilde{t}_{2}}^{2}}{m_{\tilde{t}_{1}}^{2}-m_{\tilde{t}_{2}}^{2}} \log \left(\frac{m_{\tilde{t}_{1}}^{2}}{m_{\tilde{t}_{2}}^{2}}\right)\right)\right],
\end{aligned}
$$

where $m_{t}$ is the mass of the top quark, and $m_{\tilde{t}_{1}, \tilde{t}_{2}}$ are the masses for the physical top squark eigenatates discussed in the previous section. The parameter $X_{t}$ parametrizes the mixing in the top squark sector and it is given by:

$$
X_{t}=A_{t}-\frac{\mu}{\tan \beta}
$$

The magnitude of this radiative correction can be analyzed in a particular limit. In the absence of top squark mixing, Equation (2.85) reduces to:

$$
\delta m_{h^{0}}^{2} \approx \frac{12}{16 \pi^{2}} \frac{m_{t}^{4}}{v^{2}} \log \left(\frac{m_{\tilde{t}_{1}} m_{\tilde{t}_{2}}}{m_{t}^{2}}\right) .
$$

From the above equation, one can check that to barely raise the mass of the lightest Higgs boson above the LEP bound one needs top squarks $\geq 500 \mathrm{GeV}$ for a top mass of $172.5 \mathrm{GeV}$. However, this yields one-loop corrections which happen to be 
of the same order as the tree level contribution. A more rigorous analysis will have to incorporate higher order corrections, predominantly from gluinos. They have been shown to lower the mass of the lightest Higgs [27]. Therefore, one may wish to push the mass of the top squarks to values well above $500 \mathrm{GeV}$. Of course the value of the mixing parameter, $X_{t}$ can be tuned in order to maximize the value of the Higgs mass for lower values of the top squarks masses. However, In Chapter 1, I discussed that for SUSY to be a viable solution to the hierarchy problem, the new supersymmetric partners would have to lie close to the weak scale. As the mass of the superpartners grow, fine tunings proportional to the ratio between the weak scale and the scale that parametrized SUSY, $m_{S U S Y}$ reappear. Therefore, one may ask how large of a stop mass is necessary in order to raise the mass of the lightest Higgs above the LEP bound. In particular, in the maximal mixing scenario defined by setting $A_{t}=\sqrt{6} m_{\tilde{t}}$, it has been found that $m_{h^{0}} \lesssim 135 \mathrm{GeV}$ for top squark masses close to $\sim 2 \mathrm{TeV}[27,77]$.

Recall that in a SUSY theory with soft SUSY breaking, the parameters of the theory do receive contributions from soft mass parameters. For example, the mass parameter for the Higgs, $m_{H_{u}}^{2}$ recieves a dominat contribution arising from top squark loops:

$$
\frac{d m_{H_{u}}^{2}}{d t} \approx \frac{3 y_{t}^{2}}{4 \pi^{2}} m_{\tilde{t}}^{2}
$$

where $y_{t}$ is the top Yukawa coupling and $t$ is a dimensionless energy scale. In other words, the Higgs parameter receives contributions which are proportional to $m_{\tilde{t}}$. This poses a problem for the MSSM since one of the minimization conditions for electroweak symmetry breaking states that

$$
\frac{1}{2} m_{Z}^{2}=\frac{m_{H_{d}}^{2}-m_{H_{u}}^{2} \tan ^{2} \beta}{\tan ^{2} \beta-1}-\mu^{2} .
$$


From Equation (2.89) one can check that in order to avoid any fine cancellation among parameters, the right hand side of the equation need not be orders of magnitude greater than $m_{Z}$. To minimize this fine tuning one may want to have:

$$
m_{\tilde{t}} \lesssim \sqrt{2} m_{Z} .
$$

Since $m_{Z} \sim 91 \mathrm{GeV}$, the mass of the top squark necessary to minimize the amount of fine tuning in the parameters is too small to yield an experimentally viable Higgs mass. In order to barely pass the LEP bound, one will have to incorporate cancellations which turn out to be approximately an order of magnitude greater than those parametrized by Equation (2.90). Therefore, the MSSM is already slightly fine tuned, but the situation gets worse as the mass of the Higgs increases. This tension that arises from the fine cancellation of the parameters of the MSSM is known as the little hiearchy problem. In order to relieve the amount of fine tuning one may want to find ways to raise the Higgs mass without the need of large radiative corrections. In Chapter 4, I will introduce and study a model that has been developed to yield a natural solution to the little hierarchy problem of the MSSM. 


\section{CHAPTER 3}

\section{THE NEXT-TO-MINIMAL SUPERSYMMETRIC MODEL}

In this Chapter I will review some important aspects of the NMSSM. An excellent, and more complete study is provided in Reference [62]. The NMSSM was introduced as an elegant way to solve the $\mu$-problem of the MSSM. The $\mu$ problem of the MSSM is characterized by the presence of a mass parameter in the superpotential, whose magnitude needs to be of the order of the electroweak scale and not the natural cut-off scale where gravitational quantum effects become relevant, $M_{\text {Planck }}$. Within the NMSSM, the $\mu$-term is replaced by a Yukawa coupling between $H_{u}$ and $H_{d}$ and a new complex scalar field, $S$ uncharged under the Standard Model gauge group. As this "gauge singlet", S, develops a vev of order $\sim O\left(M_{S U S Y}\right)$, a $\mu$-terms is generated, and the $\mu$-problem of the MSSM is solved dynamically.

Incorporating a gauge singlet into the Higgs sector of the MSSM has interesting phenomenological consequences. The first is an extended Higgs sector which consists now of of three CP-even and two CP-odd Higgs bosons. The modified scalar potential can yield larger masses for a SM-like Higgs boson, and new lighter states can appear providing an interesting phenomenology consisting of for exam-

ple Higgs cascade decays [25, 31, 59, 108]. Additionally, the fermionic component of $S$ mixes into the other four neutralinos of the MSSM. This is very interesting if as a result the mostly-singlet neutralino is the lightest state as well as the lightest 
supersymmetric particle (LSP). In this case, the next-to-lightest supersymmetric particle (NLSP) would not readily decay into the LSP, as a result displaced vertices can can be obeserved at colliders $[15,18,36,58,74,82,85,96]$.

I will also show in this chapter how in order to solve the $\mu$-problem of the MSSM, the NMSSM runs into a large degree of tension when also required to address the little hierarchy problem, and necessitates a certain amount of finetuning of the parameters of the model. In the next chapter I will introduce a variation of the NMSSM, in which the singlet is absolved of its responsibility for solving the $\mu$-problem. By doing so, it will be shown that a solution to the little hierarchy problem can be achieved in a more natural way.

\subsection{Higgs potential and mass matrices}

The NMSSM is characterized by a scale invariant superpotential:

$$
W=W_{\text {Yukawa }}+\lambda \hat{S} \hat{H}_{u} \hat{H}_{d}+\frac{\kappa}{3} \hat{S}^{3} .
$$

The Higgs potential is given by the SUSY gauge interactions, F-terms and soft SUSY breaking terms:

$$
\begin{aligned}
V_{H} & =\left|\lambda\left(H_{u}^{+} H_{d}^{-}-H_{u}^{0} H_{d}^{0}\right)+\kappa S^{2}\right|^{2} \\
& +\left(m_{H_{u}}^{2}+\lambda^{2}|S|^{2}\right)\left(\left|H_{u}^{0}\right|^{2}+\left|H_{u}^{+}\right|^{2}\right)+\left(m_{H_{d}}^{2}+\lambda^{2}|S|^{2}\right)\left(\left|H_{d}^{0}\right|^{2}+\left|H_{d}^{-}\right|^{2}\right) \\
& +\frac{g^{\prime 2}+g^{2}}{8}\left(\left|H_{u}^{0}\right|^{2}+\left|H_{u}^{+}\right|^{2}-\left|H_{d}^{0}\right|^{2}-\left|H_{d}^{-}\right|^{2}\right)^{2}+\frac{g^{2}}{2}\left|H_{u}^{+} H_{d}^{0 *}+H_{u}^{0} H_{d}^{-*}\right|^{2} \\
& +m_{S}^{2}|S|^{2}+\left(\lambda A_{\lambda}\left(H_{u}^{+} H_{d}^{-}-H_{u}^{0} H_{d}^{0}\right) S+\frac{1}{3} \kappa A_{\kappa} S^{3}+h . c\right)
\end{aligned}
$$


where $g^{\prime}$ and $g$ denote the $U(1)_{Y}$ and $S U(2)$ gauge couplings and $A_{\lambda}, A_{\kappa}$ and $m_{S}^{2}$ are soft terms associated with the singlet.

The neutral complex Higgs fields can be parametrized as follows:

$$
H_{u}^{0}=v_{u}+\frac{H_{u R}+i H_{u I}}{\sqrt{2}}, \quad H_{d}^{0}=v_{d}+\frac{H_{d R}+i H_{d I}}{\sqrt{2}}, \quad S=v_{S}+\frac{S_{R}+i S_{I}}{\sqrt{2}}
$$

where $v_{u}, v_{d}$ and $v_{S}$ are the vevs of the field's real components. Minimization of the Higgs scalar potential follows as in the MSSM, except that now we obtain an additional constrain arising from the vev of the real part of the singlet field. In this way, the minimization conditions for electroweak symmetry breaking are given by:

$$
\begin{gathered}
v_{u}\left(m_{H_{u}}^{2}+\mu_{e f f}^{2}+\lambda^{2} v_{d}^{2}+\frac{g^{\prime 2}+g^{2}}{4}\left(v_{u}^{2}-v_{d}^{2}\right)\right)-v_{d} \mu_{e f f} B_{e f f}=0 \\
v_{d}\left(m_{H_{d}}^{2}+\mu_{e f f}^{2}+\lambda^{2} v_{u}^{2}+\frac{g^{\prime 2}+g^{2}}{4}\left(v_{d}^{2}-v_{d}^{2}\right)\right)-v_{u} \mu_{e f f} B_{e f f}=0 \\
v_{S}\left(m_{S}^{2}+\kappa A_{\kappa} v_{S}+2 \kappa^{2} v_{S}^{2}+\lambda^{2} v^{2}-2 \lambda \kappa v_{u} v_{d}\right)-\lambda v_{u} v_{d} A_{\lambda}=0
\end{gathered}
$$

where $\mu_{e f f}=\lambda v_{S}, B_{\text {eff }}=A_{\lambda}+\kappa v_{S}$ and as in the MSSM, $v=\sqrt{v_{u}^{2}+v_{d}^{2}}=174$ $\mathrm{GeV}$ and $\tan \beta=v_{u} / v_{d}$. Using these three minimization conditions one may replace the soft mass parameters $m_{H_{u}}^{2}, m_{H_{d}}^{2}$, and $m_{S}^{2}$ for a solution to $v_{u}, v_{d}$ and $v_{S}$. In this way, one can note that a vev for the singlet is obtained of the order of the SUSY scale, generating a $\mu_{\text {eff }}$ of the same order and providing a solution to $\mu$-problem of the MSSM.

In order to study electroweak symmetry breaking in the NMSSM, one must study the conditions for an acceptable minimum. As in the MSSM, both $v_{u}$ and $v_{d}$ must not vanish, see Section 2.3.3, and $v_{S}$ must be large enough to generate an effective $\mu$-term consistent with electroweak symmetry breaking as well as bounds 
on supersymmetric particles [5]. This condition can be analyzed by inspecting the Higgs potential at large values of the singlet vev, $v_{S}$. In this limit the Higgs potential reduces to:

$$
V_{H} \sim m_{S}^{2} v_{S}^{2}+\frac{2}{3} \kappa A_{\kappa} v_{S}^{3}+\kappa^{2} v_{S}^{4}
$$

A condition for a non-zero $v_{S}$ is given by $A^{2} \gtrsim 8 m_{S}^{2}$. In addition, together with the following condition for an absolute minimum, $A_{\kappa}^{2} \gtrsim 9 m_{S}^{2}$, one obtains the following relationship between the vev of $S$ and the soft SUSY breaking parameters $[43,60]$ :

$$
v_{S} \approx \frac{1}{4 \kappa}\left(-A_{\kappa}-\sqrt{A_{\kappa}^{2}-8 m_{S}^{2}}\right)
$$

The mass matrices at tree level are obtained by expanding the Higgs potential around the vevs $v_{u}, v_{d}$ and $v_{S}$. The masses for the CP-even Higgs bosons are obtained by diagonalizing a $3 \times 3$ mass matrix in the the basis $\left(H_{d R}, H_{u R}, S_{R}\right)$. In what follows, the minimization conditions have been used to replace $m_{H_{u}}^{2}, m_{H_{d}}^{2}$, and $m_{S}^{2}$ for the vevs of the real pieces of the neutral Higgs bosons:

$$
\begin{aligned}
M_{S, 11}^{2} & =g^{2} v_{d}^{2}+\mu_{e f f} B_{e f f} \tan \beta \\
M_{S, 22}^{2} & =g^{2} v_{u}^{2}+\mu_{e f f} B_{e f f} \cot \beta \\
M_{S, 33}^{2} & =\frac{\lambda}{2} A_{\lambda} v^{2} \sin 2 \beta+\kappa v_{S}\left(A_{\kappa}+4 \kappa v_{S}\right) \\
M_{S, 12}^{2} & =\left(\lambda^{2}-\frac{g^{2}}{2}\right) \frac{v^{2} \sin 2 \beta}{v_{S}}-\mu_{e f f} B_{e f f} \\
M_{S, 13}^{2} & =\lambda\left(2 \mu_{e f f} v_{d}-\left(B_{e f f}+\kappa v_{S}\right) v_{u}\right) \\
M_{S, 23}^{2} & =\lambda\left(2 \mu_{e f f} v_{u}-\left(B_{e f f}+\kappa v_{S}\right) v_{d}\right)
\end{aligned}
$$

The upper bound on the lightest scalar, $h^{0}$ can be obtained by rotating the upper 
$2 \times 2$ submatrix of Equation (3.7) by an angle $\beta$ :

$$
m_{h^{0}}^{2} \approx m_{Z}^{2} \cos ^{2} \beta+\frac{\lambda^{2}}{2} v \sin ^{2} \beta .
$$

As can be seen from the above equation, the mass of $h^{0}$ is enhanced over the MSSM value by an amount $\frac{\lambda^{2}}{2} v^{2} \sin ^{2} \beta$. This enhancement can in principle address the little hierarchy problem, however it is only relevant for not too large values of $\tan \beta$. Furthermore, in general situations, the mass of $h^{0}$ will be smaller due to the presence of mixing between $h^{0}$ and the remaining two scalars. In order to maximize the value of $h^{0}$ it is important to minimize the mixing of the singlet, $S$ into $h^{0}$. The mixing is given in the $(1,3)$ element of Equation (3.7):

$$
-\lambda A_{\lambda} \sin 2 \beta+2 \lambda \kappa v_{S} \sin 2 \beta \approx 0,
$$

and it yields the following relation between parameters in the singlet scalar potential:

$$
A_{\lambda} \approx-2 \kappa v_{S} .
$$

From the above equation, $A_{\lambda}$ must be tuned to obtained electroweak symmetry breaking as well as a value of $m_{h^{0}}$ above the LEP bound. This tuning is also depicted in Figure 3.1, where the mass of $h^{0}$ has been plotted as a function of $A_{\lambda}$ for a typical NMSSM case where $\lambda=0.7, \kappa=0.05, M_{\tilde{g}}=500 \mathrm{GeV}$ and $m_{\tilde{t}}=1 \mathrm{TeV}$ in the maximal mixing scenario where $A_{t}=\sqrt{6} m_{\tilde{t}}$. As can be seen from the figure, there only remains a small portion of parameter space where electroweak symmetry breaking can be achieved while generating a Higgs boson with mass above the LEP bound. One can argue that the value of $\lambda$ could be made large enough to avoid the tuning in Equation (3.10) while raising the 


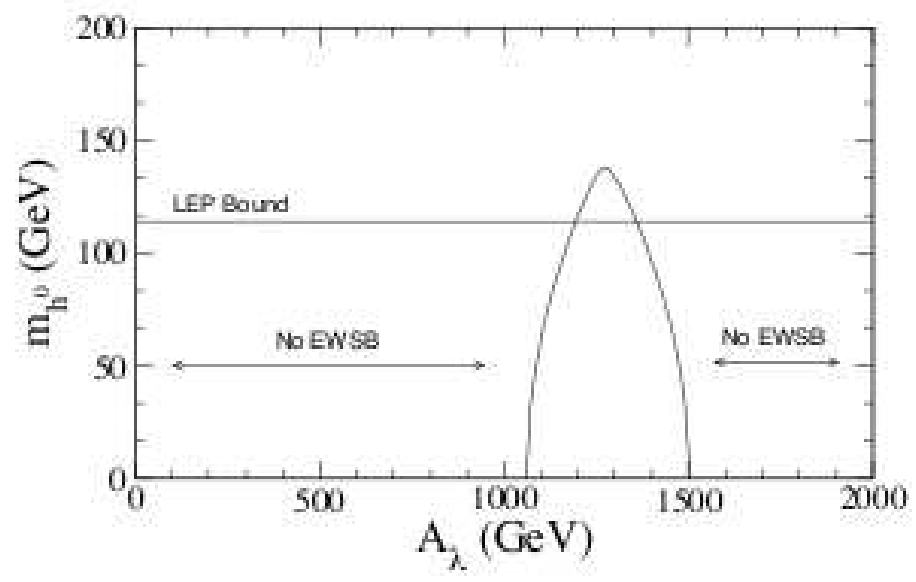

Figure 3.1. Mass of $h^{0}$ as a function of $A_{\lambda}$ for a typical NMSSM case with $\lambda=0.7, \kappa=0.05, M_{\tilde{g}}=500 \mathrm{GeV}$ and $m_{\tilde{t}}=1 \mathrm{TeV}$ in the maximal mixing scenario where $A_{t}=\sqrt{6} m_{\tilde{t}}$.

value of the Higgs mass arbitrarily above the LEP bound. However, if one insists that gauge unification is real, the value of $\lambda$ must be bounded at the electroweak scale by $\lambda\left(m_{W}\right) \lesssim 0.7$. The details on how one obtains this bound is discussed in Section 4.2.2. One is then left with a large degree of tension in trying to resolve both the $\mu$-problem together with raising the mass of the light Higgs boson above the LEP bound. This will serve as a motivation to seek a more natural solution to the little hierarchy problem and it is the main topic of this thesis which will be dealt with in full in Chapter 4.

The CP-odd spectrum is obtained by diagonalizing the following mass matrix 
in the basis $\left(H_{d, I}, H_{u I}, H_{I}\right)$ :

$$
\begin{aligned}
M_{P, 11}^{2}= & \mu_{e f f} B_{e f f} \tan \beta \\
M_{P, 22}^{2}= & \mu_{e f f} B_{e f f} \cot \beta \\
M_{P, 33}^{2}= & \lambda\left(B_{e f f}+3 \kappa v_{S}\right) \frac{v^{2} \sin 2 \beta}{2 v_{S}}-3 \kappa A_{\kappa} v_{S} \\
& M_{P, 12}^{2}=\mu_{e f f} B_{e f f} \\
& M_{P, 13}^{2}=\lambda v_{u}\left(A_{\lambda}-2 \kappa v_{S}\right) \\
& M_{P, 23}^{2}=\lambda v_{d}\left(A_{\lambda}-2 \kappa v_{S}\right)
\end{aligned}
$$

As in the MSSM, $M_{P}^{2}$ contains a massless Goldstone mode. The charged Higgs mass can be obtained by diagonalizing the following $2 \times 2$ matrix:

$$
\begin{aligned}
& M_{ \pm, 11}^{2}=\left(\mu_{e f f} B_{e f f}+\frac{v^{2} \sin 2 \beta}{2}\left(\frac{g^{2}}{2}-\lambda^{2}\right)\right) \cot \beta \\
& M_{ \pm, 22}^{2}=\left(\mu_{e f f} B_{e f f}+\frac{v^{2} \sin 2 \beta}{2}\left(\frac{g^{2}}{2}-\lambda^{2}\right)\right) \tan \beta \\
& M_{ \pm, 12}^{2}=\mu_{e f f} B_{e f f}+\frac{v^{2} \sin 2 \beta}{2}\left(\frac{g^{2}}{2}-\lambda^{2}\right) \\
& M_{ \pm, 21}^{2}=\mu_{e f f} B_{e f f}+\frac{v^{2} \sin 2 \beta}{2}\left(\frac{g^{2}}{2}-\lambda^{2}\right)
\end{aligned}
$$

As in the MSSM, the above matrix contains also a Goldstone mode. The mass of the charged Higgs is then given by:

$$
m_{H^{ \pm}}=\frac{2 \mu_{e f f} B_{e f f}}{\sin 2 \beta}+m_{W}^{2}-\lambda^{2} v^{2},
$$

where $m_{W}$ is the mass of the $W$ gauge boson as in the Standard Model. 


\subsection{Limiting cases of the NMSSM}

In this section I point out two important limits of the NMSSM which can be attained by setting some of its parameters to zero, and identifying potential global symmetries that arise in such models.

\subsubsection{The Effective MSSM}

It is clear from Equation (3.1) that if one takes $\lambda \rightarrow 0$, any coupling between the singlet and $H_{u, d}$ vanish. But given that $\mu_{e f f}=\lambda v_{S}$ should be large enough, one needs to explore Equation (3.6) for a viable decoupling between the singlet and $H_{u, d}$ sector. One way to achieve this will be to let $\kappa \rightarrow 0$ such that $v_{S} \rightarrow \infty$ while keeping all dimensionful parameters fixed. In this way, one can derive the parameters of an effective MSSM. These are given by:

$$
\begin{array}{r}
\mu_{e f f}=\lambda v_{S} \\
B_{\text {eff }}=A_{\lambda}+\kappa v_{S} .
\end{array}
$$

It would seem that in this limit, one will not be able to distinguish the effective MSSM from the MSSM. However, the fermionic component of the singlet, $\tilde{S}$, obtains a mass proportional to $\kappa v_{S}$ and can easily become the LSP $[15,18,36$, $58,74,82,85,96]$. Therefore, the phenomenology of the effective MSSM will proceed as in the MSSM, but the final decay in the cascade chain will contain a singlet-like neutralino. This final decay is proportional to $\lambda$, and will imply a very long lifetime for the singlet-like neutralino and the possibility of displaced vertices observed at collider detectors. 


\subsubsection{The Peccei-Quinn limit}

Another interesting limit of the NMSSM occurs when taking $\kappa \rightarrow 0$. In this limit, the Lagrangian of the NMSSM possesses a global symmetry given by the following transformation on the scalar Higgs fields:

$$
H_{u} \rightarrow H_{u} e^{i \phi}, \quad H_{d} \rightarrow H_{d} e^{i \phi}, \quad S \rightarrow S e^{-2 i \phi} .
$$

This symmetry is known as a Peccei-Quinn symmetry and provides a solution to the strong CP-problem [97, 98]. However, when the neutral real components of the Higgs fields get a vev, this global symmetry is spontaneously broken, and a massless Nambu-Goldstone boson, an axion $a$, appears in the spectrum. This poses a problem for the NMSSM, since setting $\kappa$ to zero may destabilize the potential, and strong constraints are placed on the parameters space of the NMSSM. One may decide to explicitly break the Peccei-Quinn symmetry by a small $\kappa$ term, making the axion massive but very light. This has interesting implications for the phenomenology of the NMSSM, since in this limit one can potentially induce branching fractions of the SM-like Higgs boson into aa larger than the branching fraction into $b \bar{b}$. This case has been studied in [55].

To summarize, I have provided a brief summary of the NMSSM and have shown where fine tuning in the parameter space arises when using the NMSSM as a model to address both the $\mu$-problem of the MSSM and the little hierarchy problem. Even though the NMSSM possesses interesting limits and symmetries with rich phenomenology, it still remains imperative to study variations of singlet extended MSSM models to find a more natural solution to the little hierarchy, while retaining the possibility of a rich phenomenology which will make this class of models interesting to study with the outgoing experimental power of the LHC. 


\section{CHAPTER 4}

\section{A NATURAL SOLUTION TO THE LITTLE HIERARCHY PROBLEM: THE S-MSSM}

In this chapter I present a generalization of the NMSSM, discussed in Chapter 3 , with an explicit $\mu$-term and a supersymmetric mass for the singlet superfield, as a route to alleviating the little hierarchy problem of the MSSM. In what follows, I study the Higgs spectrum and two limiting cases, characterized by the size of the singlet's mass [41, 42].

\subsection{Model}

The superpotential of the NMSSM is not the most general superpotential one encouters when extending the MSSM by the addition of one gauge singlet. In fact, if one impose gauge symmetries and R-parity, the most general renormalizable superpotential is:

$$
W=W_{\text {Yukawa }}+(\mu+\lambda \hat{S}) \hat{H}_{u} \hat{H}_{d}+\frac{\mu_{S}}{2} \hat{S}^{2}+\frac{\kappa}{3} \hat{S}^{3}+\xi \hat{S} .
$$

This superpotantial contains explicit mass terms both for the $H_{u} H_{d}$ pair (the usual $\mu$-term) and for the singlet itself $\left(\mu_{S}\right)$, as well as a tadpole term for the singlet and a cubic self-coupling. 
In our analysis of the S-MSSM, $\mu$ and $\mu_{S}$ will be allowed to take arbitrary values at or below the $\mathrm{TeV}$ scale, independent of the value of the vev of S. In this way, any attempt at solving the $\mu$-problem of the MSSM is not sought for. This is the key trade-off that its made in this model. A solution to the $\mu$-problem is abandoned in the hope of solving the little hierarchy problem.

Additionally, in our analysis, a bare tadpole term can be removed by field redefinitions, and even though there is no symmetry that forbids it, the nonrenormalization theorem will prevent the tadpole term form being generated radiatively until SUSY is broken. Once SUSY is broken, the tadpole could reappear, but not in a calculable way. If the tadpole coefficient $\xi \gg M_{S U S Y}$, the singlet will develop a huge vev and prevent electroweak symmetry breaking. To model properly physics at the electroweak scale, we do not consider this scenario. If in the other hand $\xi \ll M_{S U S Y}$, then the tadpole is not relevant and it can be ignored. Only when the $\xi \sim M_{S U S Y}$ is the tadpole relevant, and in order to calculate it one has to specify the mechanism of SUSY breaking. However, this in itself requires a certain amount of tuning in the parameters of the S-MSSM, hence the tadpole is not considered further.

Finally, with the cubic term, $S^{3}$, that is required in the NMSSM in order to stabilize the potential in the singlet direction, the potential in the S-MSSM is stabilized by the explicit $\mu_{S}$-term. Given that the $S^{3}$ term is no longer needed to stabilize the potential, and since its effects tend to be small, $\kappa$ will be taken to zero in the analysis that follows. Of course the analysis can be made with a non-zero $\kappa$, but not much is gained from such scenario.

After the above considerations are taken into account, the superpotential for 
the S-MSSM is given by:

$$
W=W_{\text {Yukawa }}+(\mu+\lambda S) \hat{H}_{u} \hat{H}_{d}+\frac{\mu_{S}}{2} S^{2}
$$

In the past, the S-MSSM has been studied within the framework of supersymmetric standard models with arbitrary Higgs sectors with the purpose of raising the upper bound on the light Higgs mass $[63-65,84]$. The scalar potential for the $H_{u}$, $H_{d}$ and $S$ complex scalar fields is given by the familiar $F$ - and $D$-terms, as well as the soft breaking operators. Written in full, the scalar potential is given by

$$
\begin{aligned}
V_{H} & =\left(m_{H_{u}}^{2}+|\mu+\lambda S|^{2}\right)\left|H_{u}\right|^{2}+\left(m_{H_{d}}^{2}+|\mu+\lambda S|^{2}\right)\left|H_{d}\right|^{2}+\left(m_{s}^{2}+\mu_{s}^{2}\right)|S|^{2} \\
& +\left[B_{s} S^{2}+\left(\lambda \mu_{s} S^{\dagger}+B_{\mu}+\lambda A_{\lambda} S\right) H_{u} H_{d}+\text { h.c. }\right]+\lambda^{2}\left|H_{u} H_{d}\right|^{2} \\
& +\frac{1}{8}\left(g^{2}+g^{\prime 2}\right)\left(\left|H_{u}\right|^{2}-\left|H_{d}\right|^{2}\right)^{2}+\frac{1}{2} g^{2}\left|H_{u}^{\dagger} H_{d}\right|^{2}
\end{aligned}
$$

where $g$ and $g^{\prime}$ are the Standard Model gauge couplings of $S U(2)_{W}$ and $U(1)_{Y}$.

Minimizing the potential given in Equation (4.3) with respect to $H_{u}, H_{d}$ and $S$ yields the following three constraints:

$$
\begin{gathered}
\frac{1}{2} m_{Z}^{2}=\frac{m_{H_{d}}^{2}-m_{H_{u}}^{2} \tan ^{2} 2 \beta}{\tan ^{2} \beta-1}-\mu_{e f f}^{2} \\
\sin 2 \beta=\frac{2 B_{\mu, e f f}}{m_{H_{u}}+m_{H_{d}}+2 \mu_{e f f}^{2}+\lambda^{2} v^{2}} \\
v_{S}=\frac{\lambda v^{2}}{2} \frac{\left(\mu_{s}+A_{\lambda}\right) \sin 2 \beta-2 \mu}{\lambda^{2} v^{2}+\mu_{s}^{2}+m_{s}^{2}+2 B_{s}},
\end{gathered}
$$

where $v_{S}=\langle S\rangle$ and $v_{u, d}=\left\langle H_{u, d}\right\rangle$ with $v=\left(v_{u}^{2}+v_{d}^{2}\right)^{1 / 2}=174 \mathrm{GeV}, \tan \beta=v_{u} / v_{d}$ 
and

$$
\begin{aligned}
\mu_{e f f} & =\mu+\lambda v_{s} \\
B_{\mu, e f f} & =B_{\mu}+\lambda v_{s}\left(\mu_{s}+A_{\lambda}\right) .
\end{aligned}
$$

In the subsequent sections I will present in detail two very different and interesting limits of the S-MSSM characterized by the size of the supersymmetric mass of the singlet superfield $\mu_{S}$.

\subsection{The large $\mu_{S}$ limit}

The first limit of the S-MSSM that would be analyzed is that for which the supersymmetric mass term for the singlet superfield, $\mu_{S}$, is large compared to the other mass parameters in the model [41]. In this case the vev for the singlet field, $v_{S}$, given in Equation (4.6) reduces to

$$
v_{S} \approx \frac{\lambda v^{2}}{2 \mu_{S}} \sin 2 \beta .
$$

In other words, the potential in the singlet direction becomes very steep as $\mu_{S} \rightarrow$ $\infty$, forcing the vev of $S$ to lie near the origin while decoupling the singlet from the theory. This leaves the MSSM as the effective theory. In NMSSM the singlet can only be integrated out non-supersymmetrically by taking $\left|m_{S}\right|^{2}$ large, leaving behind the MSSM plus the fermionic component of the singlet superfield. This limit has been briefly considered. In reference [54], the authors briefly consider a model like the S-MSSM, but do not examine its implications in any detail. The authors of reference [29] examine a class of models which overlaps the one considered here. Additionally, the effects of decoupling a singlet have been studied through 
incorporating higher dimensional operators in the MSSM superpotential [26, 54].

I will study the spectrum of the S-MSMM for $\mu_{S}$ on the order of a few TeV, since for $\mu_{S} \rightarrow \infty$, the effects from the singlet completely decouple and any residual effect vanishes, garnishing the MSSM and its phenomenlogy. In what follows, I will present the scalar spectrum; the derivation being similar to that followed in deriving the Higgs spectrum of the MSSM studied in Chapter 2 or the NMSSM in Chapter 3.

\subsubsection{Spectrum}

As in the NMSSM, the neutral complex Higgs fields can be parametrized as follows:

$$
H_{u}^{0}=v_{u}+\frac{H_{u R}+i H_{u I}}{\sqrt{2}}, \quad H_{d}^{0}=v_{d}+\frac{H_{d R}+i H_{d I}}{\sqrt{2}}, \quad S=v_{S}+\frac{S_{R}+i S_{I}}{\sqrt{2}},
$$

where $v_{u}, v_{d}$ and $v_{S}$ are the vevs of the field's real components. Minimizing the potential with respect to the neutral components of the complex scalar fields $H_{u}$, $H_{d}$ and $S$, the following conditions are obtained:

$$
\begin{aligned}
m_{H_{u}}^{2}+\mu^{2}+\lambda^{2} v^{2} \cos ^{2} \beta+2 \lambda \mu v_{S} & +\frac{1}{4}\left(g^{2}+g^{\prime 2}\right)\left(v_{u}^{2}-v_{d}^{2}\right) \\
& =\left(B_{\mu}+\left(\lambda \mu_{S}+\lambda A_{\lambda}\right) v_{S}\right) \cot \beta \\
m_{H_{d}}^{2}+\mu^{2}+\lambda^{2} v^{2} \sin ^{2} \beta+2 \lambda \mu v_{S} & -\frac{1}{4}\left(g^{2}+g^{\prime 2}\right)\left(v_{u}^{2}-v_{d}^{2}\right) \\
& =\left(B_{\mu}+\left(\lambda \mu_{S}+\lambda A_{\lambda}\right) v_{S}\right) \tan \beta \\
\frac{v^{2} \sin 2 \beta}{2}\left(\lambda \mu_{S}+\lambda A_{\lambda}\right)-\lambda \mu v^{2} & =\left(\mu_{S}^{2}+\lambda^{2} v^{2}+m_{S}^{2}+2 B_{S}\right) v_{S} .
\end{aligned}
$$

When these are added and subtracted, one obtains Equations (4.4) - (4.6). In the absence of explicit CP-violating phases in the Higgs sector, the physical spectrum 
of the Higgs sector includes a single charged Higgs boson $\left(H^{ \pm}\right)$, three neutral scalars $\left(h^{0}, H_{1,2}^{0}\right)$, and two neutral pseudoscalars $\left(A_{1,2}^{0}\right)$. The CP-odd spectrum is obtained by diagonalizing the following mass matrix in the basis $\left(H_{u I}, H_{d I}, S_{I}\right)$ :

$$
\begin{aligned}
& M_{11}^{2}=\left(B_{\mu}+\left(\lambda \mu_{S}+\lambda A_{\lambda}\right) v_{S}\right) \cot \beta \\
& M_{12}^{2}=B_{\mu}+\left(\lambda \mu_{S}+\lambda A_{\lambda}\right) v_{S} \\
& M_{13}^{2}=\left(-\lambda \mu_{S}+\lambda A_{\lambda}\right) v \cos \beta \\
& M_{22}^{2}=\left(B_{\mu}+\left(\lambda \mu_{S}+\lambda A_{\lambda}\right) v_{S}\right) \tan \beta \\
& M_{23}^{2}=\left(-\mu_{S}+\lambda A_{\lambda}\right) v \sin \beta \\
& M_{33}^{2}=\mu_{S}^{2}+\lambda^{2} v^{2}+m_{S}^{2}-2 B_{S} .
\end{aligned}
$$

Because the matrix has a zero eigenvalue corresponding to the longitudinal polarization of the $Z^{0}$ gauge boson, simple closed forms for the eigenvalues are easy to find. However, it is more helpful to express the eigenvalues in terms of an expansion in inverse powers of $\mu_{S}$, in which it is assumed that $\mu_{S} \gg \mu, v, A_{\lambda}$ :

$$
\begin{aligned}
& m_{A_{1}^{0}}^{2}=\frac{2 B_{\mu}}{\sin 2 \beta}+\frac{2 \lambda^{2} v^{2}}{\mu_{S}}\left(2 A_{\lambda}-\frac{\mu}{\sin 2 \beta}\right)+\ldots \\
& m_{A_{2}^{0}}^{2}=\mu_{S}^{2}+\left(2 \lambda^{2} v^{2}+m_{S}^{2}-2 B_{S}\right)+\ldots
\end{aligned}
$$

where the ellipses represent higer orders in $1 / \mu_{S}$.

The mass matrix for the charged Higgs is only a $2 \times 2$, since the singlet has 
no charged component, however it still contains singlet induced corrections:

$$
\begin{aligned}
& M_{11}^{2}=\left(B_{\mu}+\left(\lambda \mu_{S}+\lambda A_{\lambda}\right) v_{S}\right) \cot \beta+\left(m_{W}^{2}-\lambda^{2} v^{2}\right) \cos ^{2} \beta \\
& M_{12}^{2}=B_{\mu}+\left(\lambda \mu_{S}+\lambda A_{\lambda}\right) v_{S}+\frac{1}{2}\left(m_{W}^{2}-\lambda^{2} v^{2}\right) \sin 2 \beta \\
& M_{22}^{2}=\left(B_{\mu}+\left(\lambda \mu_{S}+\lambda A_{\lambda}\right) v_{S}\right) \tan \beta+\left(m_{W}^{2}-\lambda^{2} v^{2}\right) \sin ^{2} \beta
\end{aligned}
$$

where $m_{W}^{2}=g^{2} v^{2} / 2$ corresponds to the mass of the $W$ gauge boson. Besides the massless Goldstone mode, the charged eigenstate has mass:

$$
m_{H^{ \pm}}^{2}=m_{A_{1}^{0}}^{2}+m_{W}^{2}+\frac{2 \lambda^{2} v^{2}}{\mu_{S}}\left[3 A_{\lambda}+2 \mu\left(\frac{1}{\sin 2 \beta}-\frac{1}{\sin ^{2} 2 \beta}\right)\right]+\ldots
$$

Note that the value of the MSSM charged Higgs boson given in Chapter $2, m_{H^{ \pm}}^{2}=$ $m_{A_{1}^{0}}^{2}+m_{W}^{2}$, is obtained in the limit $\mu_{S} \rightarrow \infty$, and taking $A_{1}^{0}$ to be the usual $A^{0}$ of the MSSM, the heavy Higgs doublet decouples in the large $m_{A^{0}}$ limit as in the MSSM.

The mass matrix for the CP-even Higgs bosons is given by the following terms:

$$
\begin{aligned}
& M_{11}^{2}=\left(B_{\mu}+\left(\lambda \mu_{S}+\lambda A_{\lambda}\right) v_{S}\right) \cot \beta+m_{Z}^{2} \sin ^{2} \beta \\
& M_{12}^{2}=-\left(B_{\mu}+\left(\lambda \mu_{S}+\lambda A_{\lambda}\right) v_{S}\right)-m_{Z}^{2} \sin \beta \cos \beta+2 \lambda^{2} v^{2} \sin \beta \cos \beta \\
& M_{13}^{2}=2 \lambda^{2} v_{S} v \sin \beta-\left(\lambda \mu_{S}+\lambda A_{\lambda}\right) v \cos \beta+2 \lambda \mu v \sin \beta \\
& M_{22}^{2}=\left(B_{\mu}+\left(\lambda \mu_{S}+\lambda A_{\lambda}\right) v_{S}\right) \tan \beta+m_{Z}^{2} \cos ^{2} \beta \\
& M_{23}^{2}=2 \lambda^{2} v_{S} v \cos \beta-\left(\mu_{S}+\lambda A_{\lambda}\right) v \sin \beta+2 \lambda \mu v \cos \beta \\
& M_{33}^{2}=\mu_{S}^{2}+\lambda^{2} v^{2}+m_{S}^{2}+2 B_{S} .
\end{aligned}
$$

Assuming that the singlet mass is large, and the mass matrix is expanded in 
inverse powers of $\mu_{S}$, the eigenvalues take on a simple form:

$$
\begin{aligned}
m_{h^{0}}^{2} & =m_{h_{M S S M}^{0}}^{2}+\frac{\lambda^{2} v^{2}}{\mu_{S}}\left(\mu \sin 2 \beta-A_{\lambda}-\Delta\right)+\ldots \\
m_{H_{1}^{0}}^{2} & =m_{H_{M S S M}^{0}}^{2}+\frac{\lambda^{2} v^{2}}{\mu_{S}}\left(\mu \sin 2 \beta-A_{\lambda}+\Delta\right)+\ldots \\
m_{H_{2}^{0}}^{2} & =\mu_{S}^{2}+\left(2 \lambda^{2} v^{2}+m_{S}^{2}-2 B_{S}\right)+\ldots
\end{aligned}
$$

where the terms labeled "MSSM" are the scalar Higgs boson masses obtained from Equation (4.3) with $\lambda=\mu_{S}=B_{S}=m_{S}^{2}=A_{\lambda}=0$ and also discussed in Chapter 2. The mass term $\Delta$ corresponds to the $1 / \mu_{S}$ correction to the splitting of the scalar Higgs boson masses and it is given by

$$
\Delta \equiv \frac{A_{\lambda}\left(m_{Z}^{2}-m_{A_{1}^{0}}^{2}\right) \cos ^{2} 2 \beta-\mu\left(m_{A_{1}^{0}}^{2}+m_{Z}^{2}\right) \sin 2 \beta}{\sqrt{\left(m_{A_{1}^{0}}^{2}+m_{Z}^{2}\right)-4 m_{A_{1}^{0}}^{2} m_{Z}^{2}}}
$$

It is obvious from Equations (4.13), (4.14), and (4.18) - (4.20) that at very large $\mu_{S}, S \approx H_{2}^{0}+i A_{2}^{0}$, both of which have masses $\sim \mu_{S}$, and the remaining Higgs bosons behave as in the MSSM.

Is also helpful to consider the lightest Higgs mass, Equation (4.18), in the Higgs decoupling limit. This limit is attained when $m_{A_{1}^{0}}$ is taken large. The limit simplifies the expressions considerably, while also maximizing the lightest Higgs mass:

$$
\begin{aligned}
m_{h^{0}}^{2} & \approx m_{Z}^{2} \cos ^{2} 2 \beta+\frac{2 \lambda^{2} v^{2}}{\mu_{S}}\left(2 \mu \sin 2 \beta-A_{\lambda} \sin ^{2} \beta\right)-\frac{\lambda^{2} v^{2}}{\mu_{S}^{2}}\left[4 \mu\left(\mu-A_{\lambda} \sin 2 \beta\right)\right. \\
& \left.+\left(A_{\lambda}^{2}-3 \lambda^{2} v^{2}-m_{S}^{2}-2 B_{S}\right) \sin ^{2} 2 \beta+m_{Z}^{2} \sin ^{2} 2 \beta \cos ^{2} 2 \beta\right] .
\end{aligned}
$$

Here I have included both the leading and sub-leading terms in the $1 / \mu_{S}$ expansion, 
both of which are required to reproduce many of the results. It is important to emphasize that the numerical work in the following section is carried out exactly.

It is natural to compare the S-MSSM light scalar, Equation (4.22) to the expression for the lightest scalar mass in the NMSSM, introduced in Chapter 3:

$$
m_{h^{0}}^{2} \leq m_{Z}^{2} \cos ^{2} 2 \beta+\frac{\lambda^{2} v^{2}}{2} \sin ^{2} \beta
$$

Some key differences are: First, the expression in Equation (4.23) is only an upper bound. In fact, it is an eigenvalue of a $2 \times 2$ submatrix corresponding to the real components of $H_{u}$ and $H_{d}$ in the full $3 \times 3$ scalar mass matrix. Mixing of the light Higgs state with the scalar component of the singlet, $S$, suppresses its mass, and so one usually tries to tune the mixing to be very small as in the NMSSM. This was shown in Chapter 3 through the following condition:

$$
A_{\lambda} \approx \frac{2 \mu}{\sin 2 \beta}-2 \kappa v_{S} .
$$

It was found that the mass of the lightest Higgs in the NMSSM falls very rapidly as one moves away from the point described by Equation (4.24). In the S-MSSM, Equation (4.22) holds in a very large range of parameter space, as it already includes the effects of mixing at leading order in $1 / \mu_{S}$. Second, the NMSSM contribution to $m_{h^{0}}^{2}$ falls as $\sin ^{2} 2 \beta$, so the effect of the singlet drops rapidly as one increases $\tan \beta$ above one. This is unfortunate for the NMSSM, since it means that the model only provides large contributions to the lightest Higgs mass precisely when the tree-level MSSM contribution is minimized. Though the situation is similar in the S-MSSM, here there are two terms with contributions to $m_{h^{0}}^{2}$, one that falls as $\sin ^{2} 2 \beta$ as in the NMSSM, but another that falls as $\sin 2 \beta$, which 
allows for new contributions at intermediate values of $\tan \beta$, yielding potentially larger masses.

\subsubsection{Perturbativity of $\lambda$}

In principle, $\lambda$ can be increased to obtain arbitrarily large Higgs masses. However, if we take the apparent gauge unification in the MSSM as a sign that physics must remain perturbative up to scales $\sim 10^{16} \mathrm{GeV}$, denoting the grand unification scale (GUT) of the Standard Model gauge group, then at the weak scale, $\lambda$ must be bounded from above such that $\lambda \lesssim 0.7$

The renormalization group equations (RGE)s for the S-MSSM are the usual RGEs of the NMSSM, though with $\kappa=0$. The details of the S-MSSM RGEs are

included in Appendix A. However, for the purpose of this discussion, the RGE for $\lambda$ is presented here:

$$
\begin{aligned}
\frac{d \lambda}{d t} & =\frac{\lambda}{16 \pi^{2}}\left(3\left(y_{t}^{2}+y_{t}^{2}\right)+y_{\tau}^{2}+4 \lambda^{2}-\frac{3}{5} g_{1}^{2}-3 g_{2}^{2}\right) \\
& +\frac{\lambda}{\left(16 \pi^{2}\right)^{2}}\left(\frac{207}{50} g_{1}^{4}+\frac{15}{2} g_{2}^{4}+\frac{9}{5} g_{1}^{2} g_{2}^{2}+\frac{6}{5} g_{1}^{2} \lambda^{2}+\frac{6}{5} g_{1}^{2} y_{\tau}^{2}\right. \\
& +\frac{4}{5} g_{1}^{2} y_{t}^{2}-\frac{2}{5} g_{1}^{2} y_{b}^{2}+6 g_{2}^{2} \lambda^{2}+16 g_{3}^{2}\left(y_{t}^{2}+y_{b}^{2}\right)-6 y_{t}^{2} y_{b}^{2} \\
& \left.-9\left(y_{t}^{4}+y_{b}^{4}\right)-3 y_{\tau}^{4}-10 \lambda^{4}-\lambda^{2}\left(9 y_{t}^{2}+9 y_{b}^{2}+3 y_{\tau}^{2}\right)\right)
\end{aligned}
$$

where $g_{1}=\sqrt{5 / 3} g^{\prime}$ is th usual GUT-normalized hypercharge coupling, $g_{2}=g$ and $t \equiv \log (Q / G e V)$ for an arbitrary energy scale $Q$.

The leading terms in the RGE for $\lambda$ are the contribution of the top Yukawa coupling, $y_{t}$, and $\lambda$ itself:

$$
\frac{d \lambda}{d t} \approx \frac{\lambda}{16 \pi^{2}}\left(3 y_{t}^{2}+4 \lambda^{2}\right)
$$




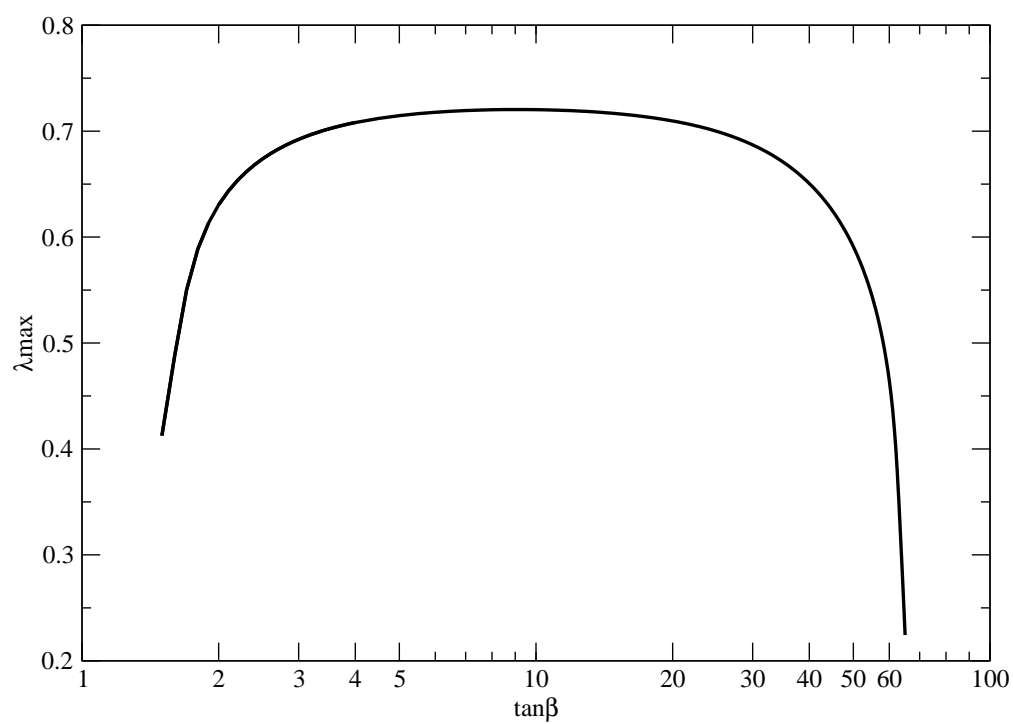

Figure 4.1. The maximum value of $\lambda$ at the weak scale, consistent with perturbativity of all couplings up to the gauge unification scale, as a function of $\tan \beta$.

Both terms tend to increase $\lambda$ at high energies and therefore the maximum value of $\lambda$ depends sensitively on the value of $y_{t}=m_{t} /(v \sin \beta)$, where $m_{t}$ is the mass of the top quark. Thus the maximum value of $\lambda$ at low energies, $\lambda_{\max }$ depends sensitively on $\tan \beta$. Here I define numerically the maximum value of $\lambda\left(m_{W}\right)$ as that value that runs to $\lambda=\pi$ at the grand unification scale.

Looking at Figure 4.1, it is seen that for $\tan \beta \approx 1$, perturbativity breaks down before the unification scale for any value of $\lambda$, but for $\tan \beta \gtrsim 1.5$, non-zero values of $\lambda$ are allowed. In fact, the maximum value of $\lambda$ rises quickly at low $\tan \beta$ until it plateus at $\lambda \approx 0.7$ for $2.5 \lesssim \tan \beta \lesssim 40$. At very high $\tan \beta$, the bottom Yukawa coupling, $y_{b}$ becomes important and $\lambda_{\max }$ is again suppressed. Throughout this work, the value of $\lambda_{\max }$ is taken to be appropriate for the value of $\tan \beta$ under consideration. 
While making use of $\lambda_{\max }$ can appear as a source of fine tuning, this is in fact natural. The value of $\lambda_{\max }$ is a low energy pseudo-fixed point of the RGEs, such that if larger $\lambda$ values are chosen at a higher energy scale, they will run down to values very close to $\lambda_{\max }$ at lower energies.

\subsubsection{Results for the large $\mu_{S}$ limit}

I now analyze the S-MSSM in order to test whether the lightest Higgs mass falls above the LEP bound of $114 \mathrm{GeV}$ while keeping a top squark spectrum below $1 \mathrm{TeV}$. Since this model has yet to be embedded into a full-blown model of SUSY breaking, it cannot spark with precision the fine tuning inherent in the calculations; but the requirement that the top squarks (and all other SUSY mass parameters) fall below a $\mathrm{TeV}$ is an often used substitute for a full fine-tuning analysis.

It is not at all obvious that the MSSM can yield large Higgs masses. This is due to the fact that for large $\mu_{S}$ the vacuum structure of the S-MSSM resembles that of the MSSM, as seen from Equation (4.9). The calculations are done using the full 1-loop effective potential descibed in Chapter 2. However, since it is well known that the 2-loop contributions can suppress the mass of the lightest Higgs, leading 2-loop contributions, dominantly from gluinos, are calculated using FeynHiggs [78]. These negative contributions are added in quadrature to the results of the 1-loop effective potential analysis. In addition, in order to maximize the light Higgs mass, $m_{A^{0}}$ is taken to be large, see Equation (4.22). The coupling $\lambda$ is also maximized following the discussion in 4.2.2.

The main result is shown in Figure 4.8, where the mass of the lightest Higgs is plotted as a function of $\tan \beta$ for a typical set of S-MSSM input paramters. For 


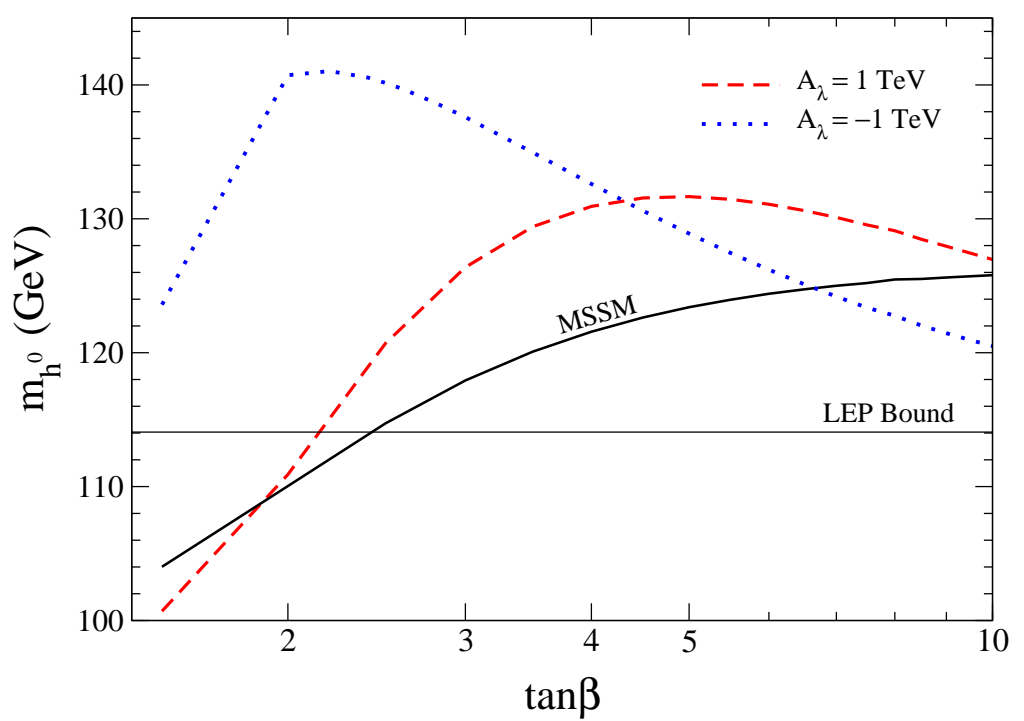

Figure 4.2. Lightest neutral Higgs mass as a function of $\tan \beta$ in the MSSM and S-MSSM. The red dashed/blue dotted curves were obtained using $\mu_{S}=m_{\tilde{t}}=M_{\tilde{g}}=1 \mathrm{TeV}$ and $A_{\lambda}= \pm 1 \mathrm{TeV}$ in the S-MSSM. The solid black curve represents the MSSM. See the text for additional parameters used in the figure.

this figure $\mu_{S}=2 \mathrm{TeV}$, a top squark mass, $m_{\tilde{t}}=1 \mathrm{TeV}$ as well as a gluino mass of $M_{\tilde{g}}=1 \mathrm{TeV}$, a $\mu$-term of $500 \mathrm{GeV}$ and $A_{\lambda}= \pm 1 \mathrm{TeV}$ on the red dashed/blue dotted lines respectively have been used. The value of $B_{S}$ has been chosen to be -(100 GeV $)^{2}$, but plays no significant role in the analysis, except in the splitting of the neutral scalars $A_{2}^{0}$ and $H_{2}^{0}$ given in Equation (4.14) and (4.20) respectively. The parameter $B_{\mu}$ is calculated as an output of the of the minimization conditions in Equations (4.4) - (4.6). In order to maximize the Higgs mass, the analysis is carried out in the "maximal mixing" case discussed in Chapter 2, where the trilinear soft-mass term in the top sector is given by $A_{t}=\sqrt{6} m_{\tilde{t}} \approx 2.5 \mathrm{TeV}$. In addition, the black dashed line represents the upper bound on the MSSM Higgs mass for the same set of input parameters. 
It is worth emphasizing the two different results depicted in the figure. First, that for which $A_{\lambda}=-1 \mathrm{TeV}$, where lightest Higgs masses above $140 \mathrm{GeV}$ are obtained at $\tan \beta \approx 2.2$ and also masses above $130 \mathrm{GeV}$ over a wide range of $\tan \beta \lesssim 5$. For $A_{\lambda}=+1 \mathrm{TeV}$, masses above $130 \mathrm{GeV}$ were also obtained but this time with larger $\tan \beta$. Second it is clear from the figure that the different sign of $A_{\lambda}$ maximizes the lightest Higgs mass at different values of $\tan \beta$. This behavior is clear from the second order terms in the $1 / \mu_{S}$ expansion of the lightest Higgs mass given in Equation (4.22).

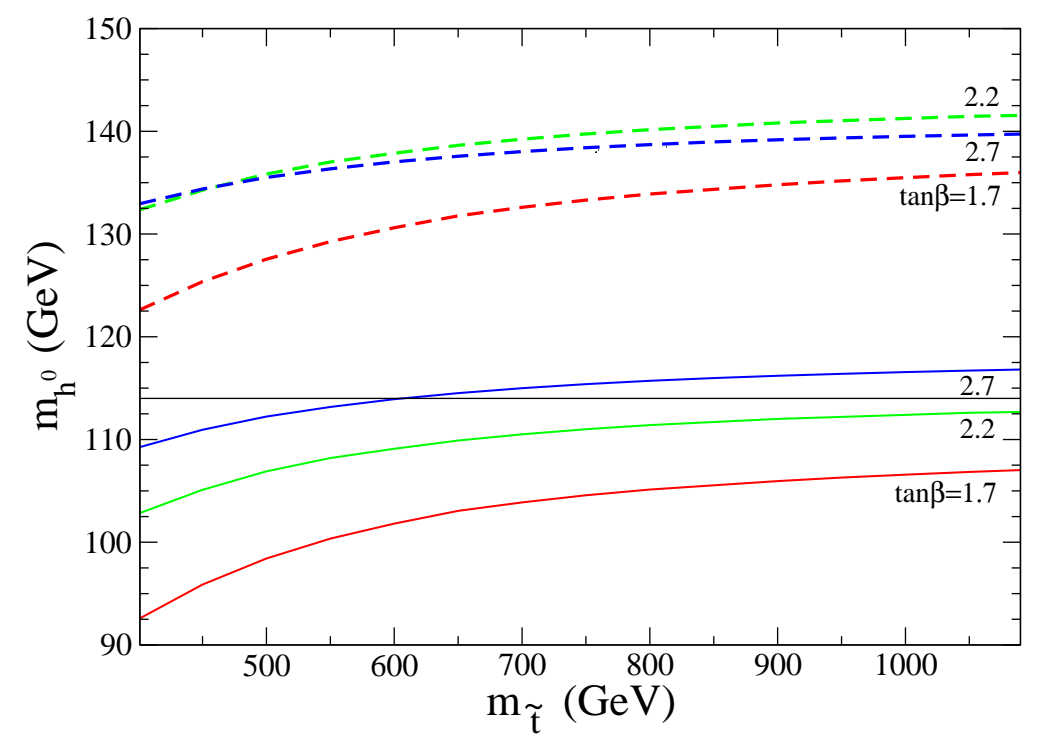

Figure 4.3. Lightest neutral Higgs mass as a function of $m_{\tilde{t}}$ in the MSSM (solid) and S-MSSM (dashed) for three values of $\tan \beta$ and assuming maximal mixing. See text for additional parameters used in the figure.

The results presented in Figure 4.8 still rely on values of the top squark which 
are rather large, that is $\sim 1 \mathrm{TeV}$. Therefore it is important to study the dependence of the lightest Higgs mass on the stop-loop induced contributions. In Figure 4.3 the dependence of the lightest Higgs mass is depicted as a function of the SUSY-breaking masses (upper dashed lines). The parameters $m_{\tilde{t}}=M_{\tilde{g}}$ are varied between $400 \mathrm{GeV}$ and $1.1 \mathrm{TeV}$ while setting $A_{t}=\sqrt{6} m_{\tilde{t}}, \mu=500 \mathrm{geV}$ and $\mu_{S}=2 \mathrm{TeV}$, for three different values of $\tan \beta$. As can be seen form the figure, even for a light spectrum, $m_{\tilde{t}} \approx 400 \mathrm{GeV}$, the mass of the lightest Higgs is well above the LEP bound of $114 \mathrm{GeV}$, whereas for the same choice of parameters the MSSM will predict masses already ruled out by experimental searches (lower solid lines).

One natural question to ask is: how can the S-MSSM be distinguished from the MSSM or the NMSSM at the LHC? For the range of parameters considered here, where $\mu_{S}$ is large compared to all other masses, the singlet-like scalars given in Equations (4.14) and (4.20) decouple, and their mixing into the lightest Higgs boson is largely suppressed. The singlet field plays a very small role in the phenomenology of the model and the dominant signature for the S-MSSM is a Higgs mass measured to be larger than allowed by the obeserved top squark mass spectrum. In all other aspects, the S-MSSM resembles the MSSM.

\subsection{The small $\mu_{S}$ limit}

In this section, the S-MSSM will be analyzed in the limit where $\mu_{S} \ll \lambda v<$ $v$ [42]. It will be seen that implementing this limit leads to a strikingly different Higgs spectrum, yet one that naturally evades the LEP bound on the Higgs mass and therefore solves the little hierarchy problem.

A couple of comments are in order about the superpotential in Equation (4.2) 
in the small $\mu_{S}$ limit. Several authors have studied a singlet-extended MSSM in the PQ limit, discussed in Chapter 3. This type of models leave behind a mass for a would-be axion. Such a model generates a $\mu$-term through the vev of the singlet field, $S$, and have no explicit $\mu_{S}$, containing an extremely light axion which corresponds to the imaginary component of the S-field. Work by $[14,79,91,107]$ studies this limit in which $\mu_{S}=\mu=0$. In the analysis presented in this section, the same class of models is examined, but the PQ-breaking soft mass terms are larger but suppressed compared to the weak scale.

In this work I consider $\mu_{S} \ll v$ and also the limit in which $m_{S}^{2}$ and $B_{S}$ are small compared to $v^{2}$ in order to simplify the calculations. These soft-breaking parameters will be put back in the numerical study. In this limit Equation (4.6) this time reduces to:

$$
v_{S} \approx \frac{1}{2 \lambda}\left(A_{\lambda} \sin 2 \beta-2 \mu\right)
$$

This immediately leads to the unexpected result where

$$
\mu_{e f f} \approx \frac{1}{2} A_{\lambda} \sin 2 \beta
$$

which is independent of $\mu$. That is, for small $\mu_{S}, B_{S}$ and $m_{S}^{2}$, the vev of the singlet, $S$, aligns in such a way as to cancel the explicit $\mu$-term completely, leaving an effective $\mu$-term which is due entirely to $A_{\lambda}$. Meanwhile, the effective $B_{\mu}$-term becomes:

$$
B_{\mu, e f f} \approx B_{\mu}+A_{\lambda}\left(\mu_{e f f}-\mu\right)
$$




\subsubsection{Spectrum}

In the absence of explicit CP-violating phases in the Higgs sector, the physical spectrum of this model includes a single charged Higgs boson $\left(H^{ \pm}\right)$, three neutral scalars which are labeled $\left(h_{S}, h, H\right)$, and two neutral pseudoscalars $\left(A_{S}, A\right)$. The states labeled with a subscript will turn out to be dominantly singlet states, while the non-subscripted states will only have a small singlet component and thus resemble their MSSM counterparts.

For the state most resembling the usual pseudoscalar Higgs, the mass is generated as in the MSSM:

$$
m_{A}^{2}=\frac{2 B_{\mu, e f f}}{\sin 2 \beta}+\ldots
$$

where $B_{\mu, e f f}$ is given in Equation (4.29). The ellipsis represents terms which are small compared to the weak scale, except when $A_{\lambda}^{2} \gg B_{\mu}, \mu^{2}$, in which case the leading correction is $\delta m_{A}^{2}=\lambda^{2} v^{2}$. It is worth noting that a $\mu_{\text {eff }} \sim O\left(m_{Z}\right)$ can be obtained by a proper choice of the parameters $A_{\lambda}, B_{\mu}$, and $\mu$ while $m_{A} \ll m_{Z}$. This class of models can reproduce the parameter space studied by the authors in references [44], in which the Higgs boson lies below the LEP bound but escapes detection since it decays dominantly into a pair of $b$-quarks.

In order to identify the mass eigenstates of the scalar Higgs boson, we must diagonalize the mass matrix given in Equation (4.17). In the small $\mu_{S}$ limit, however, it is helpful to rotate the upper $2 \times 2$ submatrix of the CP-even mass matrix by an angle $\beta$. This basis is equivalent to diagonalizing the MSSM CP-even mass matrix in the large $m_{A}$ limit. In this basis, (4.17) takes the following simple 
form

$$
M_{H}^{2}=\left(\begin{array}{ccc}
m_{Z}^{2} \cos ^{2} 2 \beta+\lambda^{2} v^{2} \sin ^{2} 2 \beta & \left(m_{Z}^{2}-\lambda^{2} v^{2}\right) \sin 2 \beta \cos 2 \beta & 0 \\
& m_{A}^{2}+\left(m_{Z}^{2}-\lambda^{2} v^{2}\right) \sin ^{2} 2 \beta & \lambda v A_{\lambda} \cos 2 \beta \\
& & \lambda^{2} v^{2}
\end{array}\right)
$$

In this basis and in the limit $\mu_{S}, m_{S}^{2}, B_{S} \ll \lambda^{2} v^{2}$, the mixing of the singlet into the lighter MSSM-like Higgs (upper diagonal component of (4.31) vanishes, yet the light Higgs still receives an NMSSM-like contribution such as that given in Equation (4.23). To leading order in $m_{Z}^{2} / m_{A}^{2}$, the light Higgs mass is simply:

$$
m_{h^{0}}^{2} \approx m_{Z}^{2} \cos ^{2} 2 \beta+\lambda^{2} v^{2} \sin ^{2} 2 \beta-\frac{\left(m_{Z}^{2}-\lambda^{2} v^{2}\right)^{2}}{m_{A}^{2}} \sin ^{2} 2 \beta \cos ^{2} 2 \beta .
$$

The last term above represents the correction from the non-decoupling of the $A$ field. The mass of the remaining neutral, MSSM-like Higgs particle is given by:

$$
m_{H}^{2} \approx m_{A}^{2}+\left(m_{Z}^{2}-\lambda^{2} v^{2}\right) \sin ^{2} 2 \beta+\frac{\left(m_{Z}^{2}-\lambda^{2} v^{2}\right)^{2}}{m_{A}^{2}} \sin ^{2} 2 \beta \cos ^{2} 2 \beta-\frac{\lambda^{2} v^{2} A_{\lambda}^{2}}{m_{A}^{2}} \sin ^{2} 2 \beta
$$

For $\lambda \gtrsim 0.5$, the scalar $H$ will fall just below the pseudoscalar $A$ in mass.

Among the states that are mostly singlet-like, there is a scalar and a pseudoscalar:

$$
\begin{aligned}
& m_{A_{S}}^{2} \approx \mu_{S}^{2}+\lambda^{2} v^{2}-\frac{\lambda^{2} v^{2} A_{\lambda}^{2}}{m_{A}^{2}} \\
& m_{h_{S}}^{2} \approx \mu_{S}^{2}-\frac{\lambda^{2} v^{2} A_{\lambda}^{2}}{m_{A}^{2}} \cos ^{2} 2 \beta .
\end{aligned}
$$

Because these states can be quite light, their explicit dependence on $\mu_{S}$ is shown. 
One point that is worth mentioning, is how the S-MSSM with small $\mu_{S}$ resembles the NMSSM where by construction has $\mu=\mu_{S}=0$. At first sight, it would seem that these two models are not very different since in the S-MSSM one can take $\mu, \mu_{S} \rightarrow 0$ and still have sizeable $\mu_{\text {eff }} \sim A_{\lambda}$. Furthermore, it is found that $\mu_{\text {eff }} \approx \frac{1}{2} A_{\lambda} \sin 2 \beta$. This is exactly the relation one requires in the NMSSM to avoid large mixing of the singlet into $h$, the Standard Model (SM)-like Higgs boson, namely Equation (4.24) in the limit $\kappa \ll 1$. In the NMSSM, this relation must hold in order to keep the mass of SM-like Higgs boson above the LEP bound, but it must be added as an additional tuning on the parameters of the model. In the S-MSSM, this relation is obtained automatically.

\subsubsection{Results for the small $\mu_{S}$ limit}

In this section, I analyze the Higgs spectrum in the small $\mu_{S}$ limit. It is examined whether it is possible to have a spectrum which naturally passes all current contraints. It is of most importance to ensure that it is possible to keep the mass of the SM-like Higgs boson above the LEP bound without requiring unnaturally large top squarks masses or mixing. A full 1-loop effective potential analysis is carried out and the leading 2-loop corrections to the mass of the SMlike Higgs boson are obtained using FeynHiggs [78]. The coupling $\lambda$ is obtained from its dependence on $\tan \beta$ as discussed in 4.2.2.

The parameter space of the model is quite different from the NMSSM. In particular, in the S-MSSM the singlet gets a vev through $A_{\lambda}$ and the soft-mass parameter $m_{S}^{2}$ is set to zero. Non-zero values are discussed after presenting the main results of this section.

One important constraint on the parameter space is the LEP bound on the 
chargino mass, $m_{\chi^{+}}>94 \mathrm{GeV}[5]$. This bound translates into a bound on $\mu_{\text {eff }}$ given by $\left|\mu_{e f f}\right|>94 \mathrm{GeV}$. Assuming small $m_{S}^{2}$, this in turn translates into a bound on $A_{\lambda}$ such that $A_{\lambda}>190 / \sin 2 \beta \mathrm{GeV}$. Therefore, for small $\tan \beta, A_{\lambda}$ is bounded from below by roughly $190 \mathrm{GeV}$; for large $\tan \beta$ the bound on $A_{\lambda}$ becomes much larger, implying a fine-tuned electroweak symmetry breaking.

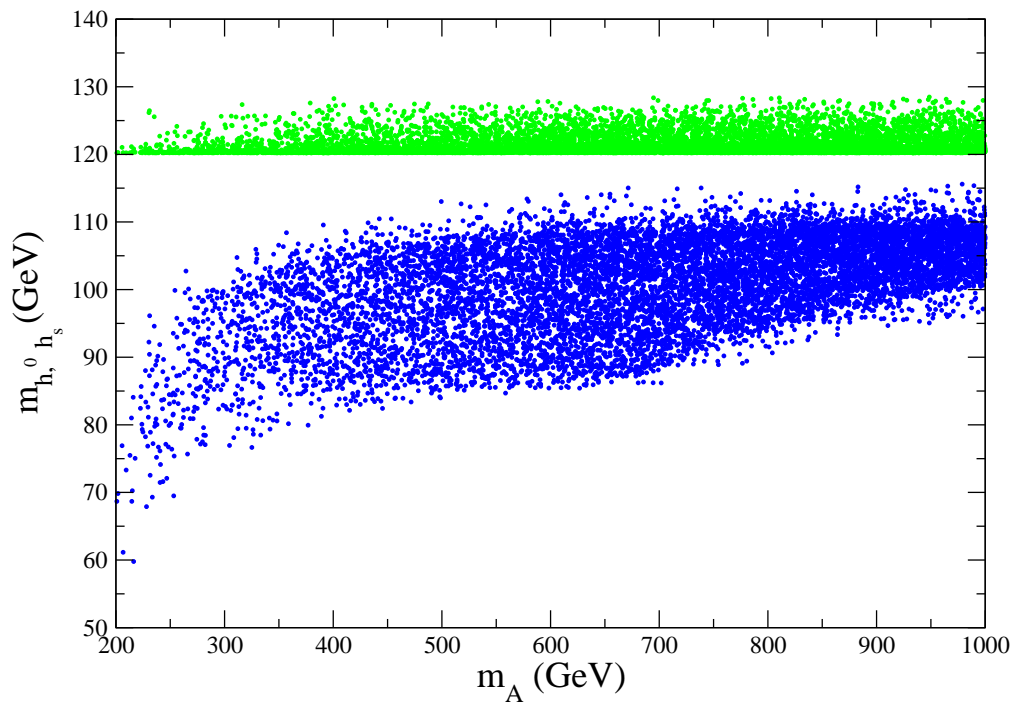

Figure 4.4. Scatter plot of $m_{h}$ (green) and $m_{h_{S}}$ (blue) as function of $m_{A}$ with a stop mass $m_{\tilde{t}}=500 \mathrm{GeV} A_{t}=0$. See text for additional parameters.

The model is examined by scanning over a wide range of parameter space with $0 \leq B_{\mu} \leq 1000^{2} \mathrm{GeV}^{2}, 0 \leq A_{\lambda} \leq 700 \mathrm{GeV}, 0 \leq \mu \leq 500 \mathrm{GeV}$, and $0 \leq \mu_{s} \leq 50$ $\mathrm{GeV}$. The parameter space is also simplified by setting $m_{S}=B_{S}=0$.

In Figure 4.4 the masses for the SM-like Higgs boson are shown together with the masses for the singlet-like scalar, $h_{S}$ as a function of the MSSM-like pseu- 


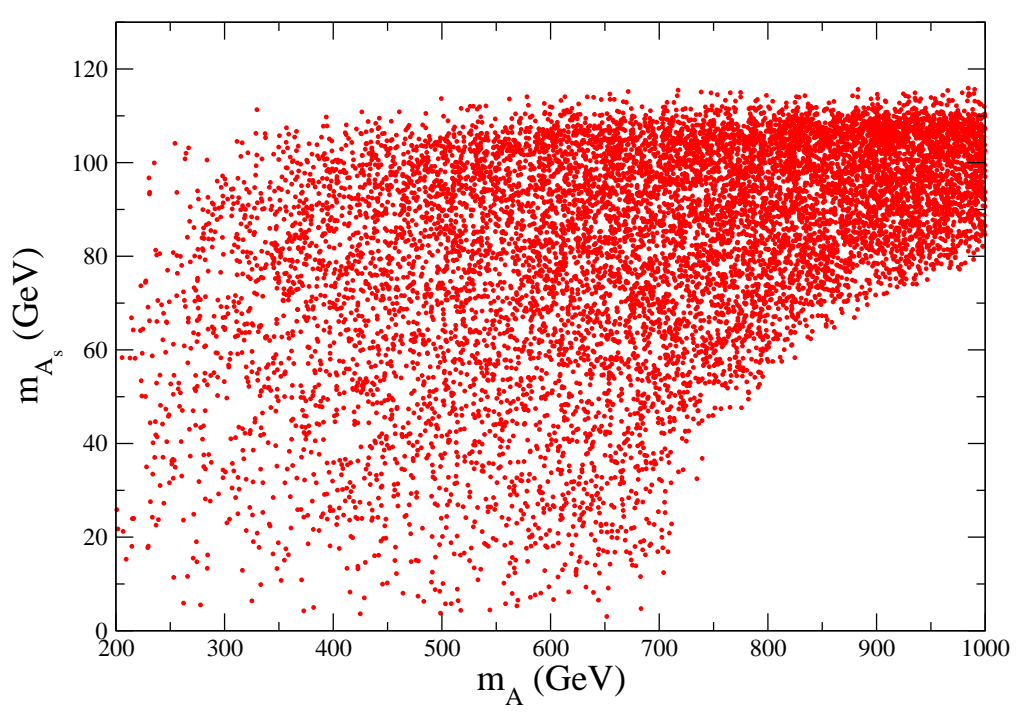

Figure 4.5. Scatter plot of $m_{A_{S}}$ (green) as a function of $m_{A}$ with a stop mass $m_{\tilde{t}}=500 \mathrm{GeV} A_{t}=0$, using the same parameter set as in Figure 4.4.

doscalar $m_{A}$ for a sample of models with $\tan \beta=2$ and $\lambda=0.63$. For this figure, I have restricted $m_{\tilde{t}}=500 \mathrm{GeV}$ and taken $A_{t}=0$, minimizing the top squark mixing. This conditions minimize the 1-loop contribution of the top squarks to the light Higgs mass and avoid any tuning arising from the top squark sector. In Figure 4.5 the corresponding masses for the singlet-like pseudoscalar, $A_{S}$, are shown. Additionally, for every point in the Figure 4.4, the masses for the singletlike states, $h_{S}$ are consistent with the LEP bound due to their reduced coupling to the $Z$ gauge boson. This is depicted in Figure 4.6 where I show a scatter plot of the SM-normalized coupling of $h_{S}$ to the $Z$ gauge boson as a function of $m_{h_{S}}$.

Looking again at Figure 4.4, one sees that, apart from a few points at low $m_{A}$, the mass of the SM-like Higgs boson is bounded from below by approximately 118 $\mathrm{GeV}$. This can be understood as it follows from Equation (4.32). For large $m_{A}$, 


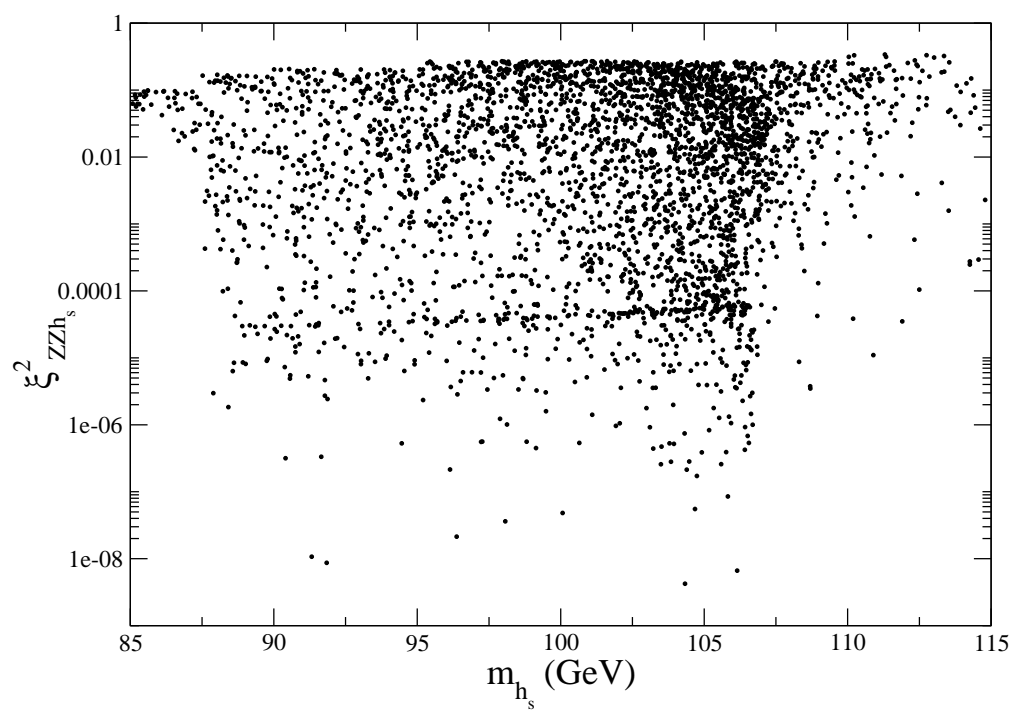

Figure 4.6. Scatter plot of the ratio $\xi^{2}=\frac{g_{h_{S} Z Z}}{g_{h Z Z}^{S M}}$ as a fnuction of $m_{h_{S}}$ using the same parameter set as in Figure 4.4.

the negative terms decouple, and the Higgs mass is bounded from below at tree level by $\left(m_{Z}^{2} \cos ^{2} 2 \beta+\lambda^{2} v^{2} \sin ^{2} 2 \beta\right)^{1 / 2}$. To this value are added one- and two-loop corrections that are nearly universal. The small amount of points scattered above the lower bound are due to the small corrections from a finite $m_{A}$, the small effects of including a non-zero $\mu_{S}$, and non-universal one-loop corrections, including those that arise from $A_{\lambda}$.

In Figure 4.7, I present the effects of varying the top squark mass and their mixing. One particular benchmark point is taken, characterized by $\mu_{S}=20 \mathrm{GeV}$, $\mu=B_{\mu}=0, A_{\lambda}=280 \mathrm{GeV}$, and $m_{S}^{2}=B_{S}=0 \mathrm{GeV}$ and for $\tan \beta=2$ and $\lambda=0.63$. The gluino an top squark masses are set to $M_{S U S Y}$ and varied from 400 to $1100 \mathrm{GeV}$. The mixing, $A_{t}$ is also varied from 0 to its maximal value of $\sqrt{6} m_{\tilde{t}}$, and I represent this range as the upper bound in the figure. In the lower bound I show the MSSM for the same choice of parameters, together with $m_{A} \rightarrow \infty$. 


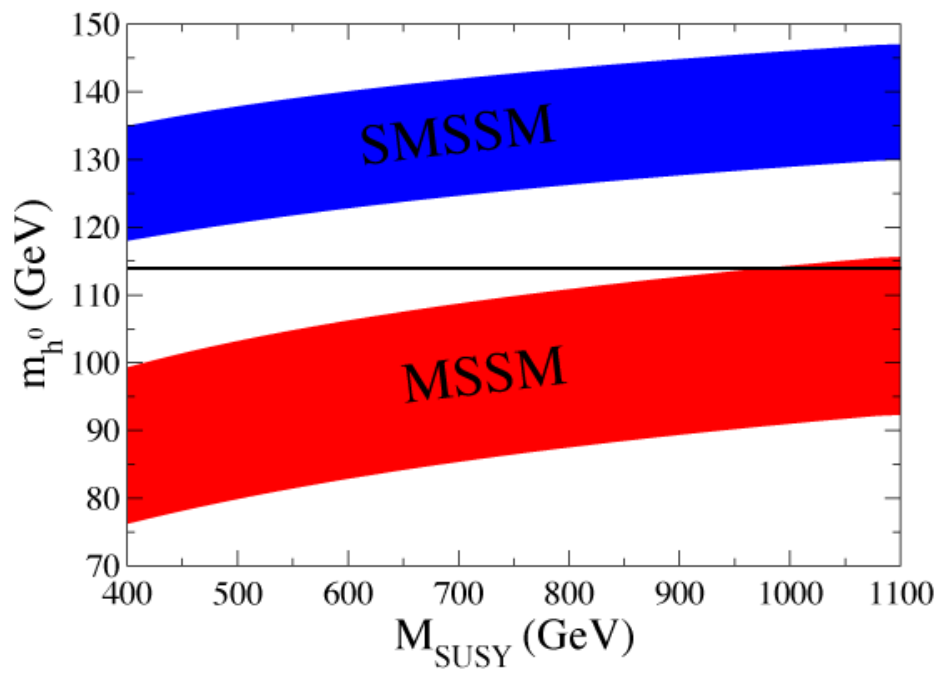

Figure 4.7. Range of mass for $h$ for the S-MSSM and the MSSM as a function of $M_{S U S Y}$. See the text for additional parameters.

As can be seen from the figure, the entire range of $M_{S U S Y}$, the S-MSSM predicts masses for SM-like Higgs boson above the LEP bound, while the MSSM can only do this with large $M_{S U S Y}$.

Finally I show a plot in which $\tan \beta$ is varied. In Figure 4.8 a scattered plot of random models is shown. The same range of parameters as for Figures $4.4-4.5$ is implemented, but in this case, $\tan \beta$ is varied using a $m_{\tilde{t}}=500 \mathrm{GeV}$ and $A_{t}=0$. The solid line represents a lower bound on the Higgs mass in all such S-MSSM models. One can immediately see that the S-MSSM automatically produces SMlike Higgs bosons with masses exceding the LEP bound for $\tan \beta \lesssim 3.8$. At $\tan \beta \gtrsim 5$, the effects of the singlet on the SM-like Higgs dissapear, and the spectrum reduces a MSSM-like spectrum.

One of the simplifications used in examining the parameter space of the S- 


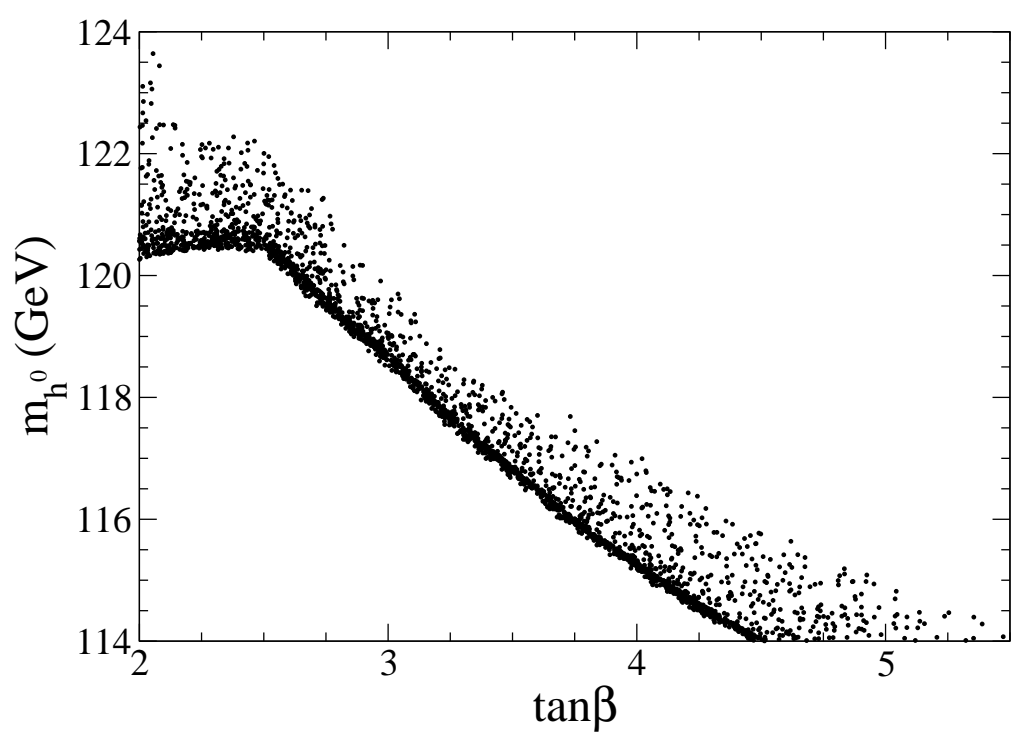

Figure 4.8. Scatter plot for $m_{h^{0}}$ as a function of $\tan \beta$ fro the same parameters used in Figure 4.4

MSSM has been to set $m_{S}^{2}=0$ throughout. However, this condition is not necessary, and in fact is somewhat unnatural. This is because there are contributions to the one-loop renormalization group equation for $m_{S}^{2}$ that are proportional to $A_{\lambda}^{2}$, and so a large $A_{\lambda}$ as it is required in this model, will tend to an equally large $m_{S}^{2}$. This can be avoided if the scale at which SUSY breaking is mediated down to the weak scale is not particularly high (More about this topic in Chapter 5.) If however one turns on $m_{S}^{2}$ such that $\epsilon=m_{S}^{2} /\left(\lambda^{2} v^{2}\right) \ll 1$, several complications arise: The first, is that the vev of $S$ shifts slightly which causes $\mu_{e f f}$ to pick up a slight dependence on the the explicit $\mu$-term:

$$
\begin{aligned}
\lambda v_{S} & \approx \frac{1}{2}(1-\epsilon)\left(A_{\lambda} \sin 2 \beta-\mu\right) \\
\mu_{e f f} & \approx \frac{1}{2}(1-\epsilon) A_{\lambda} \sin 2 \beta+\epsilon \mu .
\end{aligned}
$$


Furthermore, the exact zero in the (1,3)-element of the scalar Higgs mass matrix in Equation (4.31) is no longer zero. This element now picks up terms that scale with $\epsilon$. However this scaling is suppressed by powers of $m_{A}^{2}$, and so in the decoupling limit, the singlet again decouples from the SM-like Higgs boson:

$$
\delta m_{h}^{2} \approx\left(\frac{m_{S}^{2}}{m_{A}^{2}}\right) 2 A_{\lambda} \sin 2 \beta\left(A_{\lambda} \sin 2 \beta-2 \mu\right) .
$$

The above configuration can come with either sign, raising or lowering the mass of SM-like Higgs boson. When the SM-like Higgs is the lightest scalar in the spectrum, the mixing lowers its mass, but when the SM-like Higgs is the middle eiegenstate, mixing with $S$ can enhance its mass. In either case, the effect is small as $m_{A}$ becomes large.

In the large $\mu_{S}$ limit of the S-MSSM, discussed in Section 4.2, large Higgs masses were obtained, but the model retained a very similar phenomenology to that of the MSSM. In this limit there were not lighter singlet-like states. In the small $\mu_{S}$ limit of the S-MSSM, a number of new light states appear, which could have profound effects on the phenomenology at the LHC. One source of new phenomenology is the extended scalar sector, in particular the two light states $h_{S}$ and $A_{S}$. The $Z Z h_{S}$ coupling was calculated, as shown in Figure 4.6. As can be seen from the figure, the couplings are generically small, due to the suppressed mixing between the Higgs doublets and the singlet. This is also true for the $Z A_{S} h_{S}$ coupling as well as couplings of the $h_{S}$ and $A_{S}$ to fermions, making it difficult to produce directly at the LHC. On the other hand, there are regions of parameter space in which SM-like Higgs boson could decay dominantly into $h_{S} h_{S}$ or $A_{S} A_{S}$ pairs. This will mean that at the LHC, the predominat decay of the Higgs will be into multijets, making its discovery quite challenging but incredibly interesting. 


\subsection{Recent LHC results}

The LHC has made great strides in the search for the Standard Model Higgs boson. In 2011, the LHC delivered close to $5.6 \mathrm{fb}^{-1}$ to both the ATLAS and CMS experiments. Together with LEP and Fermilab's Tevatron, the LHC has been able to exclude a Standard Model boson up to masses of $600 \mathrm{GeV}$. The LEP collider excluded production of a Standard Model Higgs boson with mass below $114 \mathrm{GeV}[12]$ at $95 \%$ confidence level. The combined Tevatron experiments have excluded masses between 156 and $177 \mathrm{GeV}$ at 95\% confidence level [109].

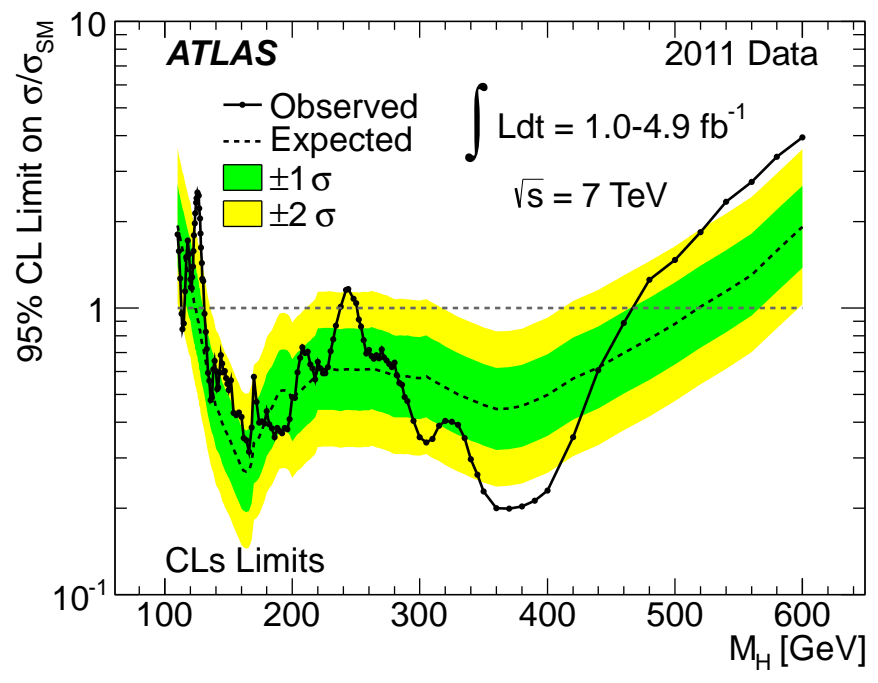

Figure 4.9. The combined 95\% CL upper limits on the signal strength as a function of $m_{h^{0}}$ measured by the ATLAS detector; the solid curve indicates the observed limit and the dotted curve illustrates the median expected limit in the absence of a signal together with the $\pm 1 \sigma$ (green) and $\pm 2 \sigma$ (yellow) bands [35]. 
The combined $95 \%$ confidence level limits on the signal strength made by the ATLAS detector using datasets corresponding to integrated luminosities from 1.04 $\mathrm{fb}^{-1}$ to $4.9 \mathrm{fb}^{-1}$ of $p p$ collisions at center of mass energies of $7 \mathrm{TeV}$ is shown in Figure 4.9. As can be seen from the figure, the mass ranges 112.9-115.5 GeV, 131-238 GeV and 251-466 GeV are excluded [35]. Similarly, the combined $95 \%$ confidence level limits on the signal strength made by the CMS detector using datasets corresponding to integrated luminosities from $4.6 \mathrm{fb}^{-1}$ to $4.8 \mathrm{fb}^{-1}$ of $p p$ collisions at center of mass energies of $7 \mathrm{TeV}$ is shown in Figure 4.10. As can be seen from the figure, the mass ranges $127-600 \mathrm{GeV}$ are excluded [34].

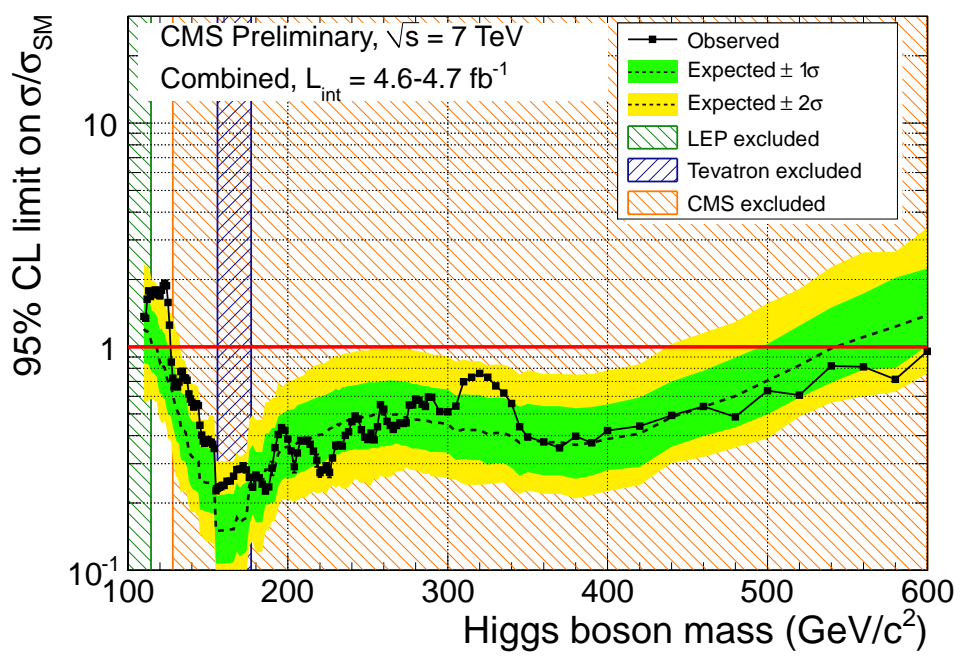

Figure 4.10. The combined 95\% CL upper limits on the signal strength as a function of $m_{h^{0}}$ measured by the CMS detector; the solid curve indicates the observed limit and the dotted curve illustrates the median expected limit in the absence of a signal together with the $\pm 1 \sigma$ (green) and $\pm 2 \sigma$ (yellow) bands [34]. 
With the new exclusions provided by the LHC, the S-MSSM is now being probed. In particular, either $\mu_{S}$ limit of the model can now be excluded if it predicts a SM-like Higgs mass $\leq 115.5 \mathrm{GeV}$ or $\geq 127 \mathrm{GeV}$. These is are great prospects for the S-MSSM since the region between 115.5 and $127 \mathrm{GeV}$ is where it predicts a SM-like Higgs consistent with the LEP bound without the need for large radiative corrections. In particular, in the small $\mu_{S}$ limit of the S-MSSM, a SM-like Higgs with mass between 116 and $126 \mathrm{GeV}$ requires a top squark with mass between 400 and $500 \mathrm{GeV}$ for a very natural choice of parameters, as seen in Figure 4.7. This is true also, for the large $\mu_{S}$ limit. The prospects are even better for the minimal implementation of GMSB in the large $\mu_{S}$ limit of the S-MSSM, where the additional structure of the model yields and upper bound for the Higgs mass of roughly $124 \mathrm{GeV}$.

To differentiate the S-MSSM from other physics beyond the Standard Model, it would be necessary to measure the properties of the SM-like Higgs boson. The situation is difficult in the large $\mu_{S}$ limit, since besides the detection of the Standard Model superpartners, the singlets are too heavy to be detected until energies are increased beyond $7 \mathrm{TeV}$ at the LHC. A SM-like Higgs boson together with top squarks between 400 and $900 \mathrm{GeV}$ would be a hint but not evidence that the S-MSSM may be the right description of physics beyond the Standard Model. The situation is much more promising in the small $\mu_{S}$ limit. A SM-like Higgs as well as a light top squark would favor the S-MSSM, but the smoking gun would rely on the observation of exotic decays of the SM-like Higgs boson into singletlike Higgs boson pairs, as $A_{S} A_{S}$ and $H_{S} H_{S}$, discussed in Section 4.3 and/or the direct detection of light singlet-like Higgs bosons. Decays of a Standard Modellike Higgs boson into singlet-like scalar pairs will not affect current LHC searches 
since at the LHC, the main production mechanism for a Higgs is through the $g g \rightarrow h \rightarrow \gamma \gamma$ channel. However, the $b \bar{b}$ channel will be largely suppressed, and this will be a a signal that the Higgs does not originate from the Standard Model. The LHC will begin colliding protons at center of mass energies of $8 \mathrm{TeV}$ this year, and it will start to probe the region left to exclude between 115.5 and $127 \mathrm{GeV}$. A SM-like Higgs boson discovered in this range will be a milestone for particle physics. Furthermore, a mass in this range will be a clear signal that the discovery of supersymmetry could be the next major breakthrough, the S-MSSM being an outstanding candidate. 


\section{CHAPTER 5}

\section{GAUGE MEDIATED SUPERSYMMETRY BREAKING}

Gauge-mediated supersymmetry breaking (GMSB) [9, 50-53, 95] is a robust scheme for generating the soft masses within low-energy models of SUSY, with a very small number of free parameters determining the entire spectrum for the model. In the following section I will introduce the principle of GMSB, but for a more complete review I refer the reader to the following review [69, 89].

\subsection{Fundamentals of GMSB}

In GMSB models, the effects of SUSY breaking are mediated through messenger fields $\Phi, \bar{\Phi}$ interacting with the MSSM through gauge interactions, thus providing a natural solution for the SUSY flavor problem [48]. This multiplet contains a messenger fermionic component, $\psi$, and a messenger scalar component, $\phi$. The messenger fields transform as a $\mathbf{5}$ and $\overline{\mathbf{5}}$ of $S U(5)$, thus preserving the appearance of gauge coupling unification that partially motivated the interest for SUSY. In GMSB theories one also implements a hidden sector whose main effects are parametrized by a chiral superfield $X$ which acquires a non-vanishing vev in both its scalar and $F$-components: that is $\langle X\rangle=M+\theta^{2} F_{X}$. In its minimal incarnation, GMSB couples the hidden sector superfield, $X$, to the messenger fields, but not to any of the usual MSSM fields. This contribution is given by the 
following superpotential:

$$
W_{\text {mess }}=y \hat{X} \hat{\Phi} \hat{\bar{\Phi}}
$$

where $y$ denotes the strength of the coupling. From the above superpotential one can obtain the mass spectrum of the messenger fermions and boson. The mass terms are given by the following Lagrangian:

$$
L=-|y\langle X\rangle|^{2}\left(|\phi|^{2}+|\bar{\phi}|^{2}\right)-\left(y\langle X\rangle \psi \bar{\psi}+y\left\langle F_{X}\right\rangle \phi \bar{\phi}+\text { h.c. }\right)
$$

The scalar messenger fields, $\phi$ and $\bar{\phi}$ obtain a mass parametrized by the following matrix:

$$
\left(\begin{array}{cc}
|y\langle X\rangle|^{2} & -y^{*}\left\langle F_{X}\right\rangle \\
-y\left\langle F_{X}\right\rangle & |y\langle X\rangle|^{2}
\end{array}\right) .
$$

Thus the scalar mass squared is obtained by diagonalizing the above matrix and it is given by: $|y\langle X\rangle|^{2} \pm|y|\left\langle F_{X}\right\rangle$, while the fermion mass is simply given by $|y|\langle X\rangle$. The fact that the fermion mass differs from the the scalar mass is an indication that the hidden sector has broken SUSY in the messenger sector.

Because the messenger fields are charged under the gauge group of the Standard Model, they will couple to both gause bosons and their superpartners, the gauginos. In this way, SUSY breaking in the messenger sector is mediated to the obeservable sector by gauge interactions at one- and -two-loops. Contributions to the gauginos masses are depicted by the 1-loop diagram in Figure 5.1. At the messenger scale, $M \equiv\langle X\rangle$, the contribution to the gaugino mass at one-loop is 


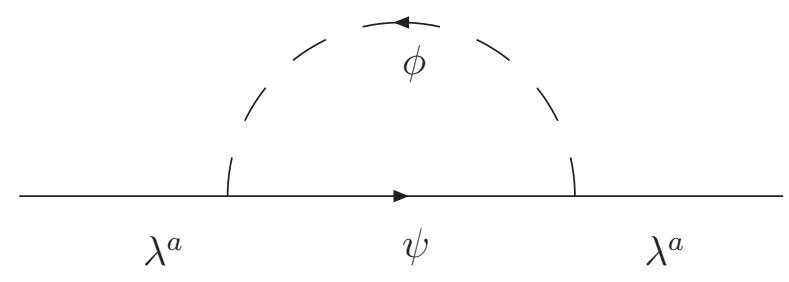

Figure 5.1. Contributions to the MSSM gaugino masses in GMSB.

given by:

$$
M_{i}(M)=n \frac{g_{i}^{2}}{16 \pi^{2}} \frac{\left\langle F_{X}\right\rangle}{\langle X\rangle},
$$

where $n$ specifies the number of messenger pairs, $i$ indexes the gauge group, and $g_{i}$ the gauge coupling. The masses for sfermions originate at two loops, with one example depicted in Figure 5.2. At the messenger scale these are given by:

$$
m_{\tilde{f}}^{2}(M)=2 n \sum_{i} C_{i}^{f} \frac{g_{i}^{4}}{\left(16 \pi^{2}\right)^{2}}\left(\frac{\left\langle F_{X}\right\rangle}{\langle X\rangle}\right)^{2}
$$

where $C_{i}^{f}$ is the quadratic Casimir associated with each gauge group, $i$, and the chiral superfield $f$. In GMSB, contributions to the trilinear soft $A$-terms appear first at two loops, while comparable mass terms, such as those in Equation (5.4) for the gauginos appear at one loop, so they can be simply set to zero at the messenger scale.

Thus far, no mention has been made of the $\mu$ and $B_{\mu}$ parameters in GMSB. There are typically two approaches that one can take. The first is to obtain $\mu$ and $B_{\mu}$ from the minimization conditions of electroweak symmetry breaking given in Equations (4.4) and (4.5), putting them into the superpotential and soft- 


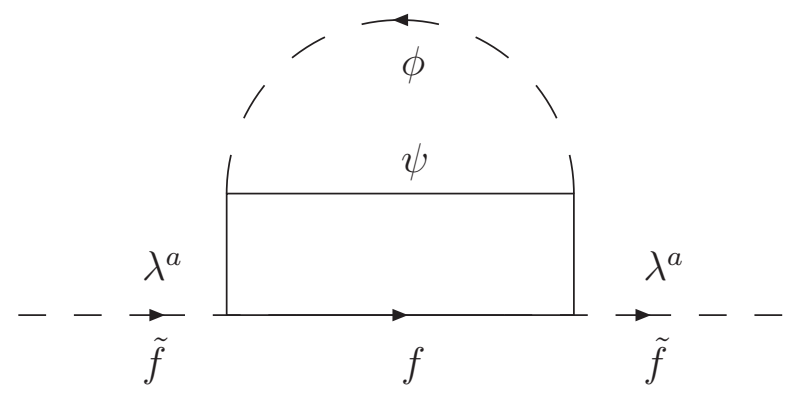

Figure 5.2. Contributions to the MSSM sfermion masses in GMSB.

breaking Lagrangian by hand. In doing this, one implicitly assumes that the physics responsible for generating $\mu$ and $B_{\mu}$ can be separated from the physics which generates the remainder of the sof-breaking terms given in Equations (5.4) and (5.5). Alternatively, one can extend the model to produce the $\mu$ and/or $B_{\mu}$ dynamically, but there are difficulties that arise which have come to be known as the $\mu / B_{\mu}$-problem of GMSB. For this reason, the first approach is taken in the study of the S-MSSM.

\subsection{GMSB in the NMSSM}

In the NMSSM, $\mu$ and $B_{\mu}$ are generated dynamically and one cannot take the first approach mentioned in the end of the previous section. Here, there is no choice but to solve the $\mu$ - and $B_{\mu}$-problems since they arise dynamically from the minimization of the scalar potential: $\mu=\lambda v_{S}$ and $B_{\mu}=\lambda A_{\lambda} v_{S}$. The literature on embedding the NMSSM into GMSB is very extensive but I will review a few ideas in order to provide a flavor of the issues and tunings that arise.

The minimal embedding of the NMSSM into GMSB in nothing more than the model described in Section 5.1. A single field $X$ communicates SUSY-breaking 


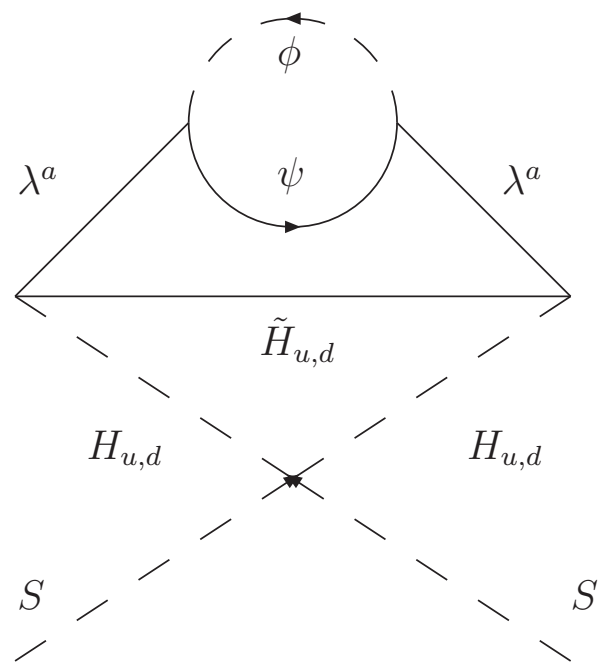

Figure 5.3. Contributions to $m_{S}^{2}$ in a minimal setup of GMSB in the NMSSM.

to a set of messengers charged under the Standard Model gauge group. The messengers then communicate SUSY-breaking to the gauginos and scalars at one and two loops respectively. The superpotential for this minimal GMSB NMSSM is given by:

$$
W=\lambda \hat{S} \hat{H}_{u} \hat{H}_{d}+\frac{1}{3} \kappa \hat{S}^{3}+\hat{X} \hat{\Phi} \hat{\bar{\phi}}
$$

Unfortunately, within this framework the singlet scalar, $S$, being neutral under the Standard Model gauge group, does not receive a soft-breaking mass, $m_{S}^{2}$, until 3-loops. This is depicted in Figure 5.3. Because $m_{S}^{2}$ sets the scale for the $S$ potential, the vev of the singlet, $v_{S}$ is suppressed. This leads to a very small dynamically generated $\mu$-term, and thus constrained by chargino and neutralino mass bounds [5].

To avoid the problem that arises from embedding the NMSSM into a minimal model of GMSB, several non-minimal embeddings have been proposed which are phenomenologically viable in at least a portion of their parameter space, and 


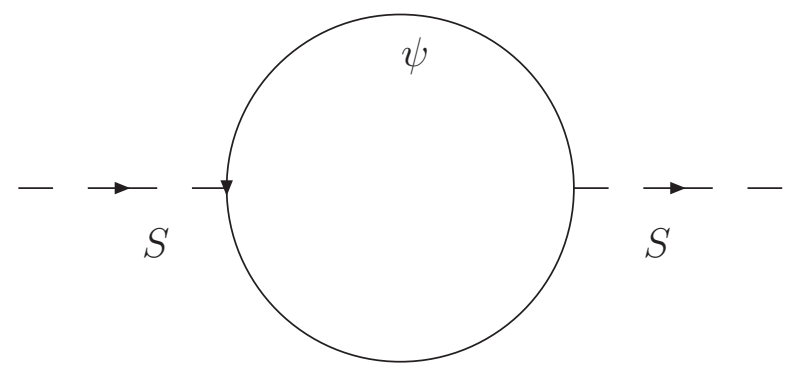

Figure 5.4. Contributions to $m_{S}^{2}$ in a setup of GMSB where the singlet superfield of the NMSSM couples to the messenger sector.

which manage to produce Higgs masses above the LEP bound. The first proposal couples the singlet $S$ directly to the messenger sector. The superpotential for this model is given by:

$$
W=\lambda \hat{S} \hat{H}_{u} \hat{H}_{d}+\frac{1}{3} \kappa \hat{S}^{3}+\hat{X} \hat{\Phi} \hat{\bar{\Phi}}-\eta \hat{S} \hat{\Phi} \hat{\bar{\Phi}}
$$

Through this framework, a soft mass-squared for the singlet, $m_{S}^{2}$, is generated at one loop, but also requires additional mass insertions of the SUSY-breaking mass scale depicted in Figure 5.4, producing a mass-squared for the singlet given by:

$$
m_{S}^{2}=\frac{\eta^{2}}{4 \pi^{2}} \frac{\left\langle F_{X}\right\rangle}{\langle X\rangle} \frac{\left\langle F_{X}\right\rangle^{2}}{\langle X\rangle^{3}} .
$$

Becasue of the additional mass insertions in the mass-squared parameter, $m_{S}^{2}$ is still too small to produce the required $\mu$-term. However, because $S$ shares the same symmetries as $X$, as seen from Equation (5.7), there can be tadpoles which are cut-off by the messenger scale and which could drive $S$ to get a large enough vev to produce the required $\mu$-term, as discussed in [61]. The possible tadpoles 


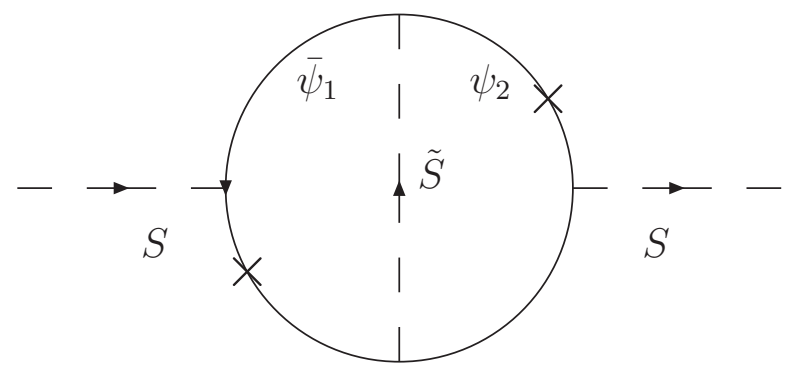

Figure 5.5. Contributions to $m_{S}^{2}$ in a setup of GMSB where the singlet superfield of the NMSSM couples to two sets of messenger fields.

are one in the superpotential, W, and one in the potential, V. They scale as:

$$
\begin{aligned}
\xi_{W} & \sim \frac{\eta}{8 \pi^{2}} \log \left(\Lambda^{2} /\langle X\rangle^{2}\right) \\
\xi_{V} & \sim \frac{\eta}{16 \pi^{2}} \frac{\langle F\rangle^{2}}{\langle X\rangle}
\end{aligned}
$$

where $\Lambda$ is some cut-off which is taken to be near the messenger scale $M \equiv\langle X\rangle$. The precise value for these tadpoles depend on physics at very high scales, but as an approximation, if $\langle F\rangle,\langle M\rangle$ and $\eta$ are chosen appropriately, both tadpoles can be of order the electroweak scale, driving $S$ to get a vev of the same size. A model of this type produces Higgs masses laying above the LEP bound either at low $\tan \beta$, large $\lambda$ and very low messenger scales, or at large $\tan \beta$ with very large messenger scales and very heavy top squarks $\left(m_{\tilde{t}} \sim 2 \mathrm{TeV}\right)$. In the first case, perturbative unification of the gauge couplings at the GUT scale, $10^{16} \mathrm{GeV}$, is not preserved without additional physics at some intermediate scale [61].

One variation on the model described above is to allow for two sets of messenger fields:

$$
W=\lambda \hat{S} \hat{H}_{u} \hat{H}_{d}+\frac{1}{3} \kappa \hat{S}^{3}+\hat{X}\left(\hat{\Phi}_{1} \hat{\bar{\Phi}}_{1}+\hat{\Phi}_{2} \hat{\bar{\Phi}}_{2}\right)-\chi \hat{S} \hat{\Phi}_{2} \hat{\bar{\Phi}}_{1}
$$


Notice here that two sets of messenger fields have been incorporated. This is a requirement enforced by the $Z_{3}$ symmetry of the NMSSM which also forbids the tadpole in $S$. The non-diagonal couplings of $S$ to the two sets of messengers prevents $m_{S}^{2}$ from being generated until two loops. This is depicted in Figure 5.5. As a consequence a $\mu$-term is generated at the right order. This model was studied in [39] where the authors found two limiting behaviors for the light Higgs mass. In one case the light Higgs mass was MSSM-like, with a tree level mass below $m_{Z}$. Here one requires large radiative corrections in order to surpass the LEP bound coming mostly from very heavy top squarks $\left(m_{\tilde{t}} \sim 2 \mathrm{TeV}\right)$. This is due primarily to the fact that in GMSB, the trilinear mass term $A_{t}$ is nowhere near its maximal value of $\sqrt{6} m_{\tilde{t}}$. In the second case, corresponding to large $\lambda, A_{\kappa}$ and $\left|m_{S}\right|^{2}$, the light Higgs mass can be raised by its mixing with the singlet, but the amount is not significant. Additionally, this case lives in a region where $\lambda$ is almost non-perturbative.

\subsection{GMSB in the S-MSSM}

For the S-MSSM, I will assume a gauge mediation sector with no direct couplings of the singlet to the messeneger fields. However, because the trilinear terms tend to be very small in GMSB, I will only be embedding the large- $\mu_{S}$ limit of the S-MSSM within this minimal GMSB framework [40]. Possible high-energy completions of the S-MSSM within the small- $\mu_{S}$ limit are a topic of future work. That being said, the superpotential for the S-MSSM within a minimal GMSB framework is given by:

$$
W=(\mu+\lambda \hat{S}) \hat{H}_{u} \hat{H}_{d}+\frac{1}{2} \mu_{S} \hat{S}^{2}+\hat{X} \hat{\Phi} \hat{\bar{\Phi}} .
$$


As noted from the previous section, the NMSSM has difficulties in this minimal implementation of GMSB because it generates too small of a soft mass for the singlet and therefore too small of a $\mu$-term. In the S-MSSM a small soft mass $m_{S}^{2}$ is not a problem since an explicit $\mu$-term is incorporated. In the analysis that follows, the values of $\mu$ and $B_{\mu}$ will be derived to obtain the correct pattern for electroweak symmetry breaking. The value of $\mu_{S}$ is taken as an input parameter. The value of $B_{S}$ plays no significant role in the model (except to provide a splitting of the mass for the singlet-like scalar and pseudoscalar) and so it is set to zero.

The procedure for finding a point in the gauge-mediated S-MSSM parameter space is as follows: I begin by choosing the messenger scale $M$. Because the contributions of supergravity mediation of SUSY-breaking [81], which scale as $F / M_{\text {Planck, }}$, are not wanted desired to compete with contributions arising from gauge mediation, the value of $M$ is chosen such that $M \leq 10^{13} \mathrm{GeV}$. For each point, a value of $\tan \beta$ is chosen, and given that value, $\lambda$ is set following the discussion of Section 4.2.2.

Given a value of $\tan \beta$, the weak-scale values for the Yukawa couplings are known. These are then run together with $\lambda$ and the gauge couplings, up to the scale $M$ using the aproppriate RGEs given in Appendix A with $\kappa=A_{\kappa}=0$. The RGEs for soft masses are given at one loop, while for dimensionless couplings these are given at two loops. At the scale $M$, the value of $\left\langle F_{X}\right\rangle$ is chosen and the soft-breaking masses for gauginos and scalars are calculated at the scale $M$ using Equations (5.4) and (5.5) for $n=1$ pair of messenger fields. The trilinear mass terms are zet to zero at the scale $M$, as well as $m_{S}^{2}$ and $B_{S}$, following the discussions in Sections 5.1 and 5.2. Given all of these boundary conditions, all masses and couplings are then run down to the weak scale. 
At the weak scale a value of $\mu$ is chosen. The S-MSSM Higgs-scalar potential is then minimized using the one-loop effective potential, solving for the electroweak symmetry breaking vev, $v$, and adjusting the value of $F$ until a value of $v=174$ is obtained. From the minimization conditions, $B_{\mu}$ and $v_{S}$ are also obtained.

The physical spectrum of the model is then calculated from the soft masses. The mass of the lightest Higgs is also corrected using the leading two loop corrections discussed in Chapter 4. For any point in parameter space, I apply all relevant experimental bounds. The most constraining bounds come from searches of neutralinos and charginos at LEP [5]. In this model, the lightest neutralino, $\chi_{1}^{0}$, turns out to be the NLSP, and it can decay into a gravitino and a photon either inside or outside the LEP detectors depending on its lifetime. For decays outside the detector, the neutralino is missing energy demanding that $m_{\chi_{1}^{0}}>46 \mathrm{GeV}$. If the decay happens inside the detector, the LEP bound on missing energy and one hard photon demands that $m_{\chi_{1}^{0}}>96 \mathrm{GeV}$. The transition from one case to another occurs at roughly $\sqrt{\langle F\rangle} \sim 10^{6} \mathrm{GeV}[69]$. This corresponds to a messenger scale of $M \sim 10^{8} \mathrm{GeV}$.

The results will be shown in several ways. First, I begin by considering two random scans of $10 \mathrm{~K}$ points, one scan each with messenger scale $M=10^{10} \mathrm{GeV}$ and $M^{13} \mathrm{GeV}$, randomly varying the other inputs witin the ranges:

$$
\begin{array}{r}
2 \leq \tan \beta \leq 6 \\
2 \leq \mu_{S} / \mu \leq 5 \\
300 \mathrm{GeV} \leq \mu \leq 900 \mathrm{GeV}
\end{array}
$$

A value for $\mu>0$ is chosen, since it is clear that that this will maximize the lightest Higgs mass (See Equation (4.22).) The upper bounds and lower bounds 


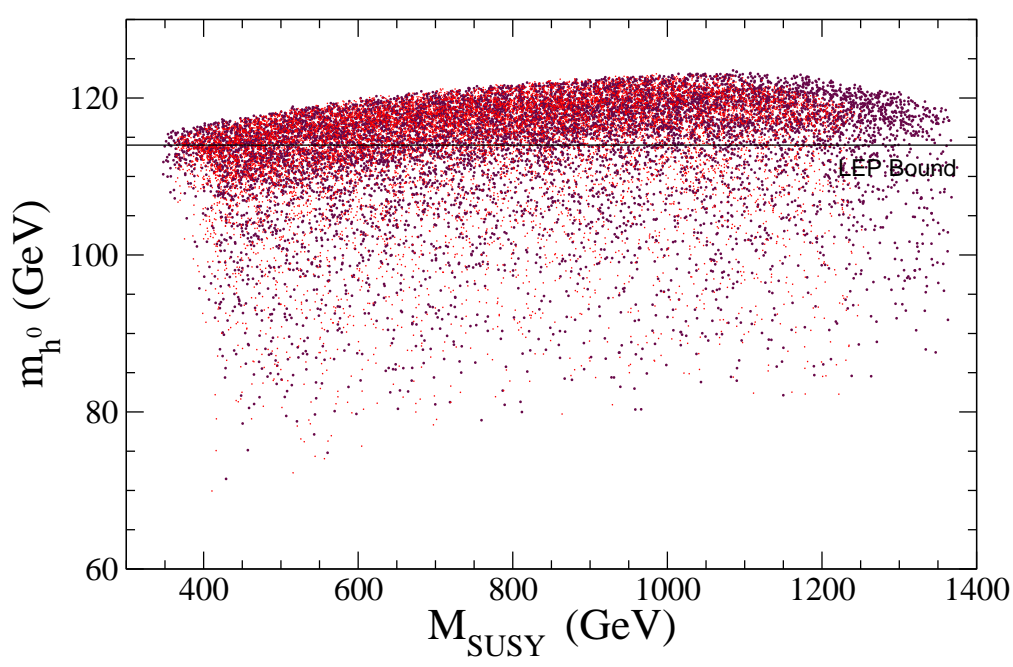

Figure 5.6. Scatterplot of the lightest Higgs boson mass versus the effective stop mass, $M_{S U S Y}$, for $10^{4}$ randomly selected points with a messenger scale $M=10^{10} \mathrm{GeV}$ (black) and $10^{13} \mathrm{GeV}$ (red), and within the parameter space given in Equation (5.13). The two regions overlapalmost exactly, except at very larg $M_{S U S Y}$. The solid black line is the LEP bound of $114 \mathrm{GeV}$.

are where the LEP bound on the neutralino begin to exclude the parameter space, and also where the top squarks are pushed above the TeV scale. The $\mu_{S}$ parameter is chosen as a multiple of $\mu$, so that $\mu_{S}$ is always large compared to other mass scales present. The points in this parameter space are chosen randomly using a flat distribution between the limits given in Equation (5.13).

Figure 5.6 shows the results of this scan. Here I plot the mass of the light Higgs boson, calculated using the leading two-loop corrections, against an effective mass parameter, $M_{S U S Y}$, defined as the geometric mean of the stop masses:

$$
M_{S U S Y} \equiv\left(m_{\tilde{t}_{1}} m_{\tilde{t}_{2}}\right)^{1 / 2}
$$

The red points have $M=10^{13} \mathrm{GeV}$, the black have $10^{10} \mathrm{GeV}$. On the plot I also 
show the LEP bound on the Standard Model Higgs of $114 \mathrm{GeV}$. It is imediately obvious that the majority of the points considered lie above the LEP bound, for either messenger scale. In fact for $M_{S U S Y} \approx 400 \mathrm{GeV}$, a large percentage of the points are already consistent with all experimental limits, and by $M_{S U S Y}=500$ $\mathrm{GeV}$, more than half the points are consistent, surpassing the LEP bound quite naturally and without any tuning.

The little difference between the messenger scales is also apparent. It appears that the results are very robust to the variation of the messenger scale. This was found to be true until the messenger scale fell below $10^{8} \mathrm{GeV}$, when the experimental bound on the neutralino became significantly stronger due to decays inside the LEP detectors.

The upper bound on the Higg mass is lower than that found in Section 4.2 where the S-MSSM was treated as an effective theory, where all parameters were chosen in order to maximize the Higgs mass. It was found that the Higgs mass could be pushed above $140 \mathrm{GeV}$. Here with the additional structure of GMSB, an upper bound on the Higgs mass of roughly $\sim 124 \mathrm{GeV}$ is found. This of course, more consistent with the more recent results from the LHC, discussed in Section 4.4.

The correlation among $\mu_{s}, M_{S U S Y}$, and the Higgs mass is shown in Figure 5.7. I plot the stop mass scale, $M_{S U S Y}$ versus $\mu_{S}$, keeping only points consistent with all experimental bounds. The points are color coded based on the calculated Higgs mass. Note that the Higgs mass constraint generates a traingular region in the figure. Note also that for very light top squarks, only a small range in $\mu_{S}$ is allowed, but as the top squarks become heavier, the range for $\mu_{S}$ grows and allow larger values of $\mu_{S}$ and heavier $h^{0}$. From the color coding one also sees how the 


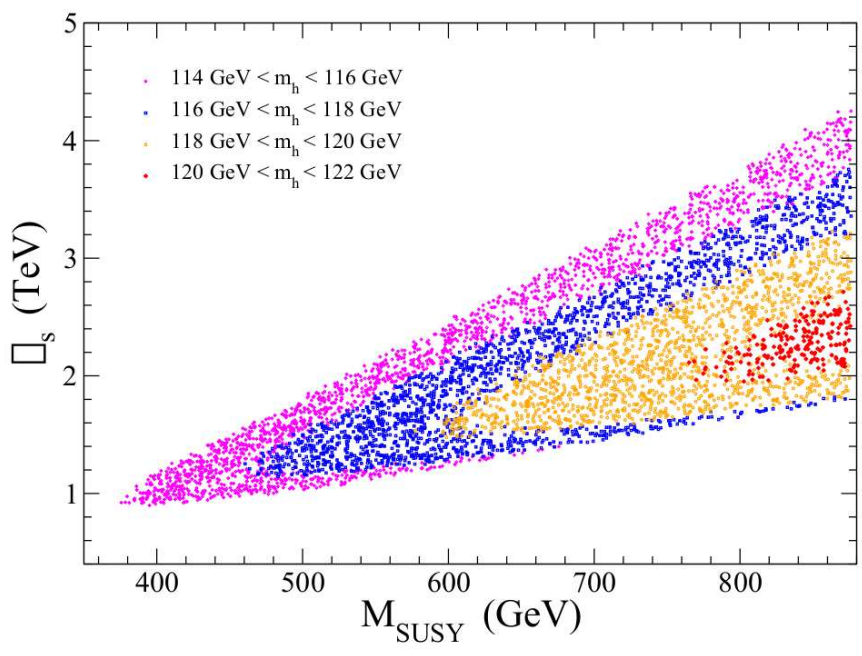

Figure 5.7. A scan of parameter space for $\tan \beta=2$ and $M=10^{13} \mathrm{GeV}$, varying $\mu$ and $\mu_{S} / \mu$ within the ranges specified in the text. All points in the figureare consistent with experimental bounds, inlcuding the bound on the Higgs mass of $114 \mathrm{GeV}$. The points are color coded by the light Higgs mass calculated from the model parameters, in $2 \mathrm{GeV}$ steps, beginning with $m_{h^{0}}<116 \mathrm{GeV}$ on the outside of the triangle and increasing to $m_{h^{0}}<118,120$ and $122 \mathrm{GeV}$ as one moves into the traingle and to the right.

Higgs mass first increases with increasing $\mu_{S}$ but then decreases as decoupling sets in.

For completeness, I have chosen a number of models which were found with scans $10^{8}, 10^{10}$ and $10^{13} \mathrm{GeV}$ and display their input parameters in Table 5.1. The masses of the top squarks and the calculated Higgs masses are also shown. The points with the heavier top squark masses just below a $\mathrm{TeV}$ were chosen to maximize the Higgs mass, and the points with lighter top squarks were chosen to minimize the top squark masses. For comparison, the mass of the lightest Higgs as predicted by the MSSM is shown using the same low energy spectrum and set 
$m_{A}=m_{A_{1}}$. From the perspective of the LHC, both of these sets of models would look identical, except for a larger Higgs mass in the S-MSSM. In particular, note from the table that all values of $m_{h^{0}}$ in the MSSM are ruled out.

Even though it is not very clear from the table, the values of the Higgs mass are not very sensitive to the change in the messenger scale $M$ for a given top squark mass. This however, breaks down at scales at or below $M=10^{8} \mathrm{GeV}$ for which the more stringent bounds on the neutralinos must be applied accounting for a neutralino decaying inside the LEP detectors.

For completeness, I take one of the points from the table (that with the lightest stops) and show its complete spectrum. Specifically for $M=10^{13} \mathrm{GeV}, \tan \beta=2$, $\mu=400 \mathrm{GeV}, \mu_{S}=1 \mathrm{TeV}$. The mass of the lightest Higgs is found at $115 \mathrm{GeV}$, and:

$$
\begin{array}{rr}
M_{1,2,3}=\{60,125,410\} \mathrm{GeV} & m_{\tilde{t}_{1,2}}=\{360,460\} \mathrm{GeV} \\
m_{\tilde{Q}_{,} \tilde{u}_{R}, \tilde{d}_{R}}=\{495,460,455\} \mathrm{GeV} \quad m_{\tilde{L}, \tilde{e}_{R}} & =\{230,130\} \mathrm{GeV} \\
m_{\chi_{i}^{0}}=\{60,11,410,420,1010\} \mathrm{GeV} & m_{\chi_{i}^{ \pm}}=\{150,595\} \mathrm{GeV} \\
m_{A_{1}^{0}, H_{1}^{0}, \pm}=\{510,515,515\} \mathrm{GeV} \quad m_{A_{2}^{0}, H_{2}^{0}}=\{1010,1010\} \mathrm{GeV}
\end{array}
$$

Notice that there are now five neutralinos, with the inclusion of the "singlino", the fermionic component of the singlet $S$. A number of sparticles in this particular spectrum are easily discovered at the LHC. However the Higgs bosons, apart from the lightest Higgs, will be a challenge for the LHC and may require a next generation lepton collider. The singlet, which is quite heavy, is well beyond any hope for discovery at the LHC. Its presence must be felt indirectly, through the mass corrections it induces in the light Higgs parametrized by the one-loop effective 
potential.

If the spectrum of quarks, sleptons, neutralinos and charginos were to be discovered at the LHC and interpreted as the MSSM, one could only predict a light Higgs mass of $74 \mathrm{GeV}$, far below the LEP bound. Even if maximal mixing had been used (large $A_{t}$ ), one could only push the mass up to roughly $102 \mathrm{GeV}$. Thus, observation of this model at the LHC, with a light Higgs mass above $102 \mathrm{GeV}$ would require physics beyond the MSSM, and the S-MSSM in the large $\mu_{S}$ limit would be a strong candidate. Unfortunately the smoking gun that could differentiate the S-MSSM from other models of new physics, the singlet, is to heavy for near discovery.

The fact that this is a model of gauge mediation does imply some other clear experimental effects. These include correlations among the sparticles an a decay of the NLSP inside the detector. From a cosmological point of view, the gravitino is the lightest SUSY particle and thus a stable dark matter candidate. That being said, the singlet sector of the large $\mu_{S}$ S-MSSM plays no role in calculating relic abundances.

It is also worth mentioning that the analysis was also carried out with $n>1$ pairs of messeneger fields, and found no significant difference between those cases and the $n=1$ case discussed in this section. 
TABLE 5.1

A SAMPLING OF POINTS WITH HIGGS MASSESS ABOVE THE LEP

BOUND.

\begin{tabular}{|c|c|c|c||c|c||c|}
\hline$M$ & $\tan \beta$ & $\mu$ & $\mu_{S}$ & $m_{\tilde{t}_{1,2}}$ & $m_{h^{0}}$ & $m_{h^{0}}(\mathrm{NMSSM})$ \\
\hline \hline $10^{8}$ & 2 & 600 & 1500 & 790,920 & 123 & 86 \\
\hline $10^{8}$ & 6 & 500 & 3000 & 760,910 & 117 & 110 \\
\hline $10^{10}$ & 2 & 350 & 875 & 350,435 & 115 & 73 \\
\hline $10^{10}$ & 3 & 300 & 1125 & 360,560 & 115 & 89 \\
\hline $10^{10}$ & 4 & 350 & 875 & 350,435 & 115 & 99 \\
\hline $10^{13}$ & 2 & 400 & 1000 & 360,460 & 115 & 74 \\
\hline $10^{13}$ & 2 & 850 & 2125 & 710,960 & 123 & 85 \\
\hline $10^{13}$ & 2.5 & 750 & 2060 & 730,960 & 124 & 94 \\
\hline $10^{13}$ & 3 & 700 & 2450 & 730,970 & 123 & 100 \\
\hline $10^{13}$ & 6 & 400 & 2400 & 470,630 & 114 & 107 \\
\hline $10^{13}$ & 6 & 600 & 3600 & 720,940 & 118 & 111 \\
\hline
\end{tabular}

Parameters are chosen to maximize $m_{h^{0}}$ or to maximize the stop masses.

All masses are in $\mathrm{GeV}$. 


\section{CHAPTER 6}

\section{THE FORWARD-BACKWARD TOP ASYMMETRY}

The CDF and D $\varnothing$ collaborations have recently reported a large forwardbackward asymmetry in the $t \bar{t}$ system which deviates from the next to-leading

order QCD standard model prediction. In particular, after unfolding they have found $[2,3]$

$$
\begin{aligned}
& A_{F B}^{t \bar{t}}=0.158 \pm 0.072 \pm 0.017\left(\mathrm{CDF} \text { with } 5.3 \mathrm{fb}^{-1}\right), \\
& A_{F B}^{t \bar{t}}=0.196 \pm 0.060_{-0.026}^{+0.018}\left(\mathrm{D} \varnothing \text { with } 5.4 \mathrm{fb}^{-1}\right),
\end{aligned}
$$

which is to be compared to the Standard Model (SM) prediction of $0.058 \pm 0.009$. Furthermore, the CDF collaboration has measured this asymmetry for different regions of $|\Delta y|$, the difference in the pseudo-rapidities of the top and anti-top quarks,

$$
\begin{aligned}
& A_{F B}^{t \bar{t}}(|\Delta y|<1)=0.026 \pm 0.118, \\
& A_{F B}^{t \bar{t}}(|\Delta y| \geq 1)=0.611 \pm 0.256 .
\end{aligned}
$$


In addition, the CDF collaboration provides a measurement of the asymmetry for two different regions of the $t \bar{t}$ invariant mass distribution:

$$
\begin{aligned}
& A_{F B}^{t \bar{t}}\left(M_{t \bar{t}}<450 \mathrm{GeV} / \mathrm{c}^{2}\right)=-0.116 \pm 0.153, \\
& A_{F B}^{t \bar{t}}\left(M_{t \bar{t}} \geq 450 \mathrm{GeV} / \mathrm{c}^{2}\right)=0.475 \pm 0.114 \text {. }
\end{aligned}
$$

Equation (1.6) has a significance of 3.1 standard deviations from the SM prediction of $0.088 \pm 0.013$. The $\mathrm{D} \emptyset$ collaboration, however, does not find a significant dependence of $A^{t \bar{t}}$ on either $|\Delta y|$ or $M_{t \bar{t}}$.

I study the asymmetry in the $t \bar{t}$ system within the framework of singlet extensions of the MSSM. For this purpose, I introduce non-renormalizable couplings between the first and third generation of quarks to scalars and analyze the two limiting cases of the model, characterized by the size of the supersymmetric mass for the singlet superfield [37]. I study both the small and large limits of this mass parameter and find that in the region of small singlet supersymmetric mass one can obtain a large asymmetry while being consistent with limits on the $t \bar{t}$ production cross section. These results are also consistent with constraints arising from flavor physics, quark masses and top quark decays as well as collider constraints.

\subsection{Model}

Within the minimal incarnation of the S-MSSM, there is no significant contribution from the Higgs spectrum to $q \bar{q}$ scattering. Therefore, in this work I consider a simple extension of this scenario by introducing the following dimension-five operators in the superpotential:

$$
W=W_{\mathrm{S}-\mathrm{MSSM}}+\frac{\Lambda_{i j}}{M} \hat{S} \hat{H}_{u} \hat{u}_{i}^{c} \hat{Q}_{j}-\frac{\Sigma_{i j}}{M} \hat{S} \hat{H}_{d} \hat{d}_{i}^{c} \hat{Q}_{j}
$$


These interactions allow for $t$-channel contributions to $q \bar{q}$ scattering mediated by Higgs particles. In particular, off-diagonal elements coupling first and third families will be relevant in generating the forward-backward asymmetry of the $t \bar{t}$ pair. The scale $M$ dictates where these operators arise, and in this work I assume it is not far from the $\mathrm{TeV}$ scale.

In light of the results from both the $\mathrm{CDF}$ and $\mathrm{D} \emptyset$ collaborations, only couplings between first and third generation of quarks will be considered. I will assume a fermion basis where all the SM up-type Yukawa couplings are diagonal before electroweak symmetry breaking. In such a basis I consider the following structure for the $\Lambda$ matrix:

$$
\Lambda=\left(\begin{array}{ccc}
0 & 0 & \Lambda_{13} \\
0 & 0 & 0 \\
\Lambda_{31} & 0 & 0
\end{array}\right) .
$$

Furthermore, I assume that $\Sigma_{i j} \approx 0$, effectively yielding no new physics contributions to the top forward-backward asymmetry. At any rate, compared to the $\Lambda$ effects, the corrections from the $\Sigma$ couplings are suppressed since these enter in the asymmetry and cross section through $d \bar{d}$ scattering.

The operators in the Lagrangian, derived from Equation (6.7), that couple first generation up quarks to their third generation counterparts through the exchange of neutral scalar or pseudoscalar Higgs bosons are given by

$$
\mathcal{L}_{u, t} \supset \sum_{i}\left(F_{R, H}^{i} H_{i}-i F_{R, A}^{i} A_{i}\right) \bar{u}_{L} t_{R}+\left(F_{L, H}^{i} H_{i}+i F_{L, A}^{i} A_{i}\right) \bar{u}_{R} t_{L}+\text { h.c. }
$$


where

$$
\begin{aligned}
F_{R,(H, A)}^{i} & =\frac{\Lambda_{31}}{\sqrt{2} M}\left(v \sin \beta O_{i, S}^{(H, A)}+v_{s} O_{i, H_{u}}^{(H, A)}\right), \\
F_{L,(H, A)}^{i} & =\frac{\Lambda_{13}}{\sqrt{2} M}\left(v \sin \beta O_{i, S}^{(H, A)}+v_{s} O_{i, H_{u}}^{(H, A)}\right) .
\end{aligned}
$$

The matrices $O^{(H, A)}$ diagonalize the scalar weak eigenstates $\left(H_{d}, H_{u}, S\right)$ into the corresponding mass eigenstates. These are labeled as $\left(H_{1}, H_{2}, H_{3}\right)$ for scalars, and $\left(A_{1}, A_{2}\right)$ for pseudoscalars in order of increasing mass. The operators coupling down quarks to top quarks through the exchange of a charged Higgs boson are given by

$$
\mathcal{L}_{d, t} \supset-\frac{v_{s}}{M} \Lambda_{31} \cos \beta \bar{d}_{L} t_{R} H^{-}+\text {h.c. } .
$$

The Feynman diagrams corresponding to the new physics in Equations (6.9) and (6.11) are shown in Figure 6.1.
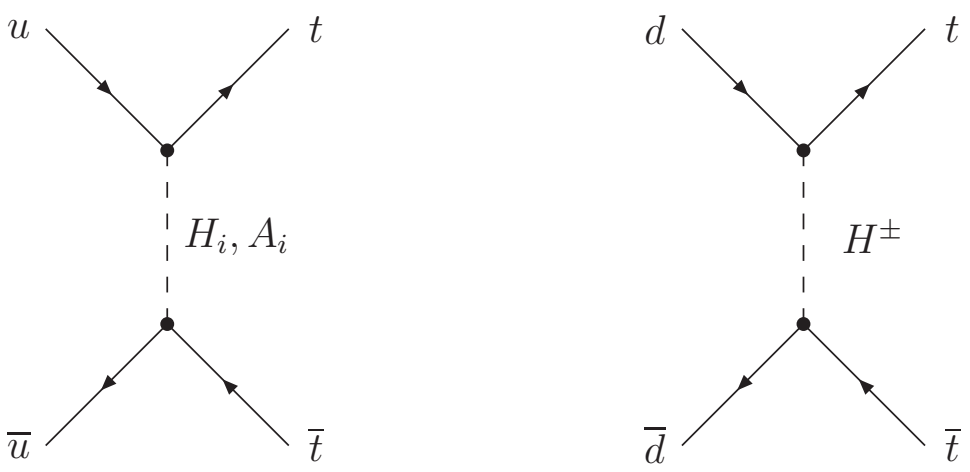

Figure 6.1. New diagrams contributing to $t \bar{t}$ production 
I will probe the parameter space of the model mainly as a function of the the singlet's vev, $v_{s}$ and the new couplings $\Lambda_{13}$ and $\Lambda_{31}$, always requiring that their values remain below $4 \pi$. Notice however, that for such large values of the couplings, extra contributions coming from higher dimensional operators could be of similar size as those given in Equation (6.7). For simplicity, in this work I restrict the study to a dimension-five analysis.

\subsection{Differential Cross Section and Asymmetry}

Following the analysis carried out by the authors in [24] the differential cross section at the parton level can be written as

$$
\frac{d \hat{\sigma}}{d \cos \theta}=M^{S M}+M^{I N T}+M^{N P},
$$

where $M_{I N T}$ denotes the interference between the SM and contributions arising from the operators given in (6.9) and (6.11), while $M_{S M}$ and $M_{N P}$ denote the contributions solely from the SM and new physics, respectively. In what follows, only the interference between new physics and the leading-order standard model diagrams will be considered; I will not incorporate the interference with the dominant NLO QCD corrections. $M_{S M}$ does include next-to-leading order contributions, and so I define the total new physics contributions by

$$
M_{\text {total }}^{N P}=M^{N P}+M_{I N T}^{S M L O, N P} .
$$


Integrating Equation (6.12) in both the forward and backward regions, one can express the asymmetry simply as:

$$
A_{F B}^{\text {total }}=A_{F B}^{N P} \cdot R+A_{F B}^{S M} \cdot(1-R)
$$

where the following definitions heve been made:

$$
\begin{aligned}
A_{F B}^{N P} & =\frac{\sigma_{F}^{N P}-\sigma_{B}^{N P}}{\sigma_{F}^{N P}+\sigma_{B}^{N P}}, \\
A_{F B}^{S M} & =\frac{\sigma_{F}^{S M}-\sigma_{B}^{S M}}{\sigma_{F}^{S M}+\sigma_{B}^{S M}}, \\
R & =\frac{\sigma_{\text {total }}^{N P}}{\sigma_{\text {total }}^{S M}+\sigma_{\text {total }}^{N P}} .
\end{aligned}
$$

The new physics contributions to the differential cross section given in Equation (6.12) can be calculated from Equations (6.9) and (6.11). The new physics $t$-channel contributions to the $t \bar{t}$ production cross section, originating from a $u \bar{u}$ initial state and mediated by scalar and pseudoscalar particles, are given by

$$
\begin{aligned}
M^{N P}(u \bar{u} \rightarrow t \bar{t}) & =\frac{\pi \beta_{t}\left(\hat{t}-m_{t}^{2}\right)^{2}}{2(16 \pi)^{2} \hat{s}} \\
& \cdot \sum_{i j}\left[\frac{A^{i j}}{\left(\hat{t}-m_{H_{i}}^{2}+i m_{H_{i}} \Gamma\left(m_{H_{i}}\right)\right)\left(\hat{t}-m_{H_{j}}^{2}-i m_{H_{j}} \Gamma\left(m_{H_{j}}\right)\right)}\right. \\
& +\frac{B^{i j}}{\left(\hat{t}-m_{A_{i}}^{2}+i m_{A_{i}} \Gamma\left(m_{A_{i}}\right)\right)\left(\hat{t}-m_{A_{j}}^{2}-i m_{A_{j}} \Gamma\left(m_{A_{j}}\right)\right)} \\
& \left.+\left(\frac{C^{i j}}{\left(\hat{t}-m_{H_{i}}^{2}+i m_{H_{i}} \Gamma\left(m_{H_{i}}\right)\right)\left(\hat{t}-m_{A_{j}}^{2}-i m_{A_{j}} \Gamma\left(m_{A_{j}}\right)\right)}+\text { h.c. }\right)\right],
\end{aligned}
$$

where $\beta_{t}=\sqrt{1-\frac{4 m_{t}^{2}}{\hat{s}}}$ and the expressions for the coefficients $A^{i j}, B^{i j}$ and $C^{i j}$ are 
given by

$$
\begin{aligned}
A^{i j} & =\left(\left(F_{R, H}^{i}+F_{L, H}^{i}\right)^{2}\left(F_{R, H}^{j}+F_{L, H}^{j}\right)^{2}+\left(F_{R, H}^{i}-F_{L, H}^{i}\right)^{2}\left(F_{R, H}^{j}-F_{L, H}^{j}\right)^{2}\right. \\
& \left.+2\left(F_{R, H}^{i 2}-F_{L, H}^{i 2}\right)\left(F_{R, H}^{j 2}-F_{L, H}^{j 2}\right)\right) \\
B^{i j} & =\left(\left(F_{R, A}^{i}+F_{L, A}^{i}\right)^{2}\left(F_{R, A}^{j}+F_{L, A}^{j}\right)^{2}+\left(F_{R, A}^{i}-F_{L, A}^{i}\right)^{2}\left(F_{R, A}^{j}-F_{L, A}^{j}\right)^{2}\right. \\
& \left.+2\left(F_{R, A}^{i 2}-F_{L, A}^{i 2}\right)\left(F_{R, A}^{j 2}-F_{L, A}^{j 2}\right)\right), \\
C^{i j} & =\left(\left(F_{R, H}^{i}+F_{L, H}^{i}\right)^{2}\left(F_{R, A}^{j}-F_{L, A}^{j}\right)^{2}+\left(F_{R, H}^{i}-F_{L, H}^{i}\right)^{2}\left(F_{R, A}^{j}+F_{L, A}^{j}\right)^{2}\right. \\
& \left.+2\left(F_{R, H}^{i 2}-F_{L, H}^{i 2}\right)\left(F_{R, A}^{j 2}-F_{L, A}^{j 2}\right)\right) .
\end{aligned}
$$

The contribution arising from a $d \bar{d}$ initial state is mediated by the charged Higgs scalar and it is given by

$$
M^{N P}(d \bar{d} \rightarrow t \bar{t})=\frac{\pi \beta_{t}\left(\hat{t}-m_{t}^{2}\right)^{2}}{2(8 \pi)^{2} \hat{s}} \frac{F_{H^{ \pm}}^{4}}{\left(\hat{t}-m_{H^{ \pm}}^{2}\right)^{2}+m_{H^{ \pm}}^{2} \Gamma^{2}\left(m_{H^{ \pm}}\right)},
$$

where $F_{H^{ \pm}}=\frac{v_{s}}{M} \Lambda_{31}$. Finally, the interference between the new physics diagrams with those arising from the leading-order QCD contribution are given by

$$
\begin{aligned}
M^{I N T}(u \bar{u} \rightarrow t \bar{t}) & =\frac{\alpha_{s} \beta_{t}}{36 \hat{s}^{2}} \sum_{i}\left(\frac{\left(F_{R,(H, A)}^{i 2}+F_{L,(H, A)}^{i 2}\right)\left(\hat{s} m_{t}^{2}+\left(\hat{t}-m_{t}^{2}\right)^{2}\right)}{\hat{t}-m_{(H, A)_{i}}^{2}+i m_{(H, A)_{i}} \Gamma\left(m_{(H, A)_{i}}\right)}\right. \\
& \left.+\frac{\left(F_{R,(H, A)}^{i 2}+F_{L,(H, A)}^{i 2}\right)\left(\hat{s} m_{t}^{2}+\left(\hat{t}-m_{t}^{2}\right)^{2}\right)}{\hat{t}-m_{(H, A)_{i}}^{2}-i m_{(H, A)_{i}} \Gamma\left(m_{(H, A)_{i}}\right)}\right),
\end{aligned}
$$

for scalar/pseudoscalar mediation and

$$
\begin{aligned}
M^{I N T}(d \bar{d} \rightarrow t \bar{t}) & =\frac{\alpha_{s} \beta_{t}}{36 \hat{s}^{2}} \frac{F_{H^{ \pm}}^{2}\left(\hat{s} m_{t}^{2}+\left(\hat{t}-m_{H^{ \pm}}^{2}+i m_{H^{ \pm}}\right)^{2}\left(m_{H^{ \pm}}\right)\right.}{} \\
& +\frac{F_{H^{ \pm}}^{2}\left(\hat{s} m_{t}^{2}+\left(\hat{t}-m_{t}^{2}\right)^{2}\right)}{\hat{t}-m_{H^{ \pm}}^{2}-i m_{H^{ \pm}} \Gamma\left(m_{H^{ \pm}}\right)}
\end{aligned}
$$


for charged scalar mediation.

\subsection{Constraints}

\subsection{1 $u-t$ Mass Mixing}

Assuming the fermion basis and the structure of $\Lambda$ introduced in Section 4.1, the operators in the Lagrangian coupling first generation to third generation up quarks are given by

$$
\mathcal{L}_{u t} \supset \frac{\Lambda_{31}}{M} S H_{u}^{0} \bar{t}_{R} u_{L}+\frac{\Lambda_{13}}{M} S H_{u}^{0} \bar{u}_{R} t_{L}+\text { h.c. } .
$$

Expanding around fluctuations from the minima of both the singlet and the uptype neutral Higgs, contributions to the masses of the up and the top quarks arise. In particular, these lead to mixing terms parametrized by the following mass matrix:

$$
M_{U}^{2}=\left(\begin{array}{cc}
\left(\Lambda_{13} \frac{v_{s} v_{u}}{M}\right)^{2} & \left(\Lambda_{13} \frac{v_{s} v_{u}}{M}\right) m_{t, 0} \\
\left(\Lambda_{13} \frac{v_{s} v_{u}}{M}\right) m_{t, 0} & \left(\Lambda_{31} \frac{v_{s} v_{u}}{M}\right)^{2}+m_{t, 0}^{2}
\end{array}\right) .
$$

In the above expression the contribution to the up-quark mass from the Yukawa sector has been taken to zero. Furthermore, I use $m_{t, 0}$ to denote the contribution

from the Yukawa sector to the top quark mass. For $m_{t, 0} \gg \Lambda \frac{v_{s} v \sin \beta}{M}$ the following are good approximations to the masses of the quark mass eigenstates

$$
\begin{aligned}
& m_{u}^{2} \approx \frac{\left(\Lambda_{31} \Lambda_{13} \frac{v_{s}^{2} v^{2} \sin ^{2} \beta}{M^{2}}\right)^{2}}{\left(m_{t, 0}^{2}+\left(\Lambda_{13} \frac{v_{s} v_{u}}{M}\right)^{2}+\left(\Lambda_{31} \frac{v_{s} v_{u}}{M}\right)^{2}\right)}, \\
& m_{t}^{2} \approx\left(m_{t, 0}^{2}+\left(\Lambda_{13} \frac{v_{s} v_{u}}{M}\right)^{2}+\left(\Lambda_{31} \frac{v_{s} v_{u}}{M}\right)^{2}\right) .
\end{aligned}
$$


Within this limit one can can see that $m_{t, 0} \approx m_{t}$. The value of $m_{t, 0}$ is then found by imposing that $m_{t} \equiv 172.5 \mathrm{GeV}$. Experimental constraints on the mass of the up quark [94] give a range of allowed values for $m_{u}$

$$
1.3 \mathrm{MeV} \leq m_{u} \leq 3.1 \mathrm{MeV}
$$

Imposing $m_{u} \leq 3.1 \mathrm{MeV}$ constrains the product of $\Lambda_{13} \cdot \Lambda_{31}$. One can impose that both couplings be small but that will generate no new physics contributions to the forward-backward top asymmetry. One can then impose that either coupling be small enough to satisfy the constraint in Equation (6.24) while making the other provide the new physics to generate a large asymmetry. In what follows, it will seen that flavor constraints will constrain $\Lambda_{13}$ over $\Lambda_{31}$.

\subsubsection{Meson mixing}

Due to the flavor mixing structure of the matrix $\Lambda$ introduced in Equation (6.9), contributions to meson mixing will arise. The operators in the Lagrangian contributing to $K^{0}-\bar{K}^{0}$ mixing are given by

$$
\mathcal{L}_{\text {mixing }} \supset-\frac{v_{s}}{M} O_{22}^{H^{ \pm}} \bar{d}_{L i}\left(V^{\dagger} \Lambda\right)_{i j} u_{R j} H^{-}+\text {h.c. },
$$

where $V$ is the CKM matrix. The above contribution to meson mixing has the same structure as that recently studied in [21]. In the model considered here the

flavor-changing matrix has an additional suppression given by $\frac{v_{s}}{2 M} O_{22}^{H^{ \pm}}$, and thus it is constrained such that

$$
\frac{1}{32 \pi^{2}}\left(\frac{\mathrm{TeV}}{m_{H^{ \pm}}}\right)^{2} \sum_{i} F\left(x_{i}\right)\left(V^{\dagger} \Lambda^{\prime}\right)_{1 i}^{2}\left(V^{\dagger} \Lambda^{\prime}\right)_{2 i}^{* 2}<10^{-6}
$$


where $x_{i}=\frac{m_{u_{i}}^{2}}{m_{H^{ \pm}}^{2}}$ and $\Lambda^{\prime}=\frac{v_{s}}{2 M} O_{22}^{H^{ \pm}} \Lambda$. The loop function $F$ is given by

$$
F(x)=\frac{1-x^{2}+2 x \log (x)}{(1-x)^{3}}
$$

Suppressing contributions to $K^{0}-\bar{K}^{0}$ mixing can be achieved with large charged Higgs masses or in the limit where $\Lambda_{13} \ll 1$.

\subsubsection{New Top decay channels}

In Chapter 4 I introduced the Higgs spectrum of the S-MSSM. In particular, in the Higgs decoupling limit of the model where $\mu_{s}$ corresponds to the largest scale in the Higgs sector, one light scalar exists and can be identified with the SM-like Higgs boson. In the small $\mu_{s}$ limit, two additional singlet-like scalars with masses below $100 \mathrm{GeV}$ are present. Due to the new flavor-changing neutral current operators present in the model, the light scalars contribute to the decay width of the top quark. In particular, for $m_{\phi_{i}} \leq m_{t}$ :

$$
\Gamma\left(t \rightarrow \phi_{i} u\right)=\frac{m_{t}}{32 \pi}\left(1-\frac{m_{\phi_{i}}^{2}}{m_{t}^{2}}\right)^{2}\left(F_{L}^{i 2}+F_{R}^{i 2}\right)
$$

where $\phi_{i}$ denotes any scalar or pseudoscalar that can be produced by a decaying top. A direct measurement of the top decay width has been carried out recently and yields an upper bound on the total decay width of the top quark of $7.6 \mathrm{GeV}$ at the $95 \%$ confidence level, for a top mass of $172.5 \mathrm{GeV}$ [1]. I incorporate this constraint to place bounds on the allowed size for the couplings $F_{L, R}$. 


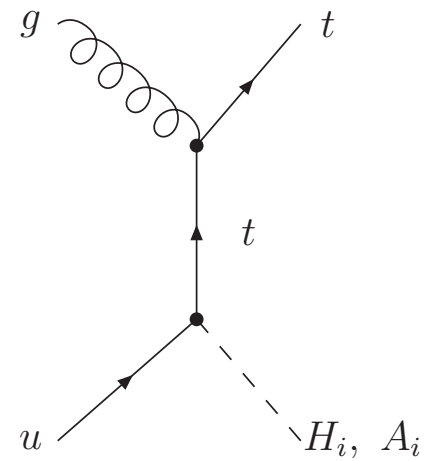

(a)

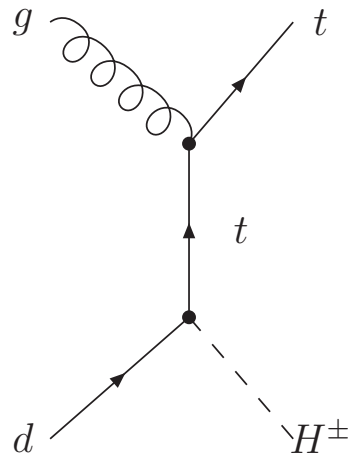

(b)

Figure 6.2. New physics diagrams contributing to single top production together with a neutral Higgs in (a) and charged Higgs in (b).

\subsubsection{Constraints from single and same-sign top production}

It is also worth mentioning the collider constraints from single top production and same-sign top production that may restrict the parameter space. In particular, from [6] it is seen that the coupling which enters into the cross section for same sign-top production is given by

$$
g_{t t} \propto\left(\Lambda_{13} \Lambda_{31}\right)^{2}
$$

Therefore, one may suppress any additional contributions to the same-sign top production cross section by suppressing one of the couplings as in the $u-t$ mass mixing constraint.

The diagrams contributing to single top production are shown in Figure 6.2. $\mathrm{D} \varnothing$ has a recent model independent measurement on the single top production cross section using center of mass energies of $\sqrt{s}=1.96 \mathrm{TeV}$ with $5.4 \mathrm{fb}^{-1}$ [4]. They find:

$$
\sigma(p \bar{p} \rightarrow t q b+X)=2.90 \pm 0.59 \mathrm{pb}
$$

for a top mass of $172.5 \mathrm{GeV}$. The diagram in Figure $6.2 \mathrm{~b}$ will contribute to the 
single top production cross section for $H^{ \pm} \rightarrow \bar{b} u$. This contribution can be suppressed by either suppressing $\Lambda_{13}$ which comes into the $d t H^{ \pm}$vertex or by a suppression of $\Lambda_{31}$ which has the effect of making the decay $H^{ \pm} \rightarrow \bar{b} u$ negligible. For a very heavy scalar or pseudoscalar the diagram of Figure $6.2 \mathrm{a}$ will be naturally suppressed at the Tevatron. For light scalars/pseudoscalars, the only decay channel open is into $b \bar{b}$, in which case the signal will be $t+2 b$ jets. In some cases there are cascade decays between Higgses, which may suppress the branching ratio into $b \bar{b}$ significantly, and the main signal will be $t+4 b$ jets. The coupling at the $\operatorname{tu}\left(H_{i} A_{i}\right)$ vertex is proportional to $F_{L, R}$ in Equation (6.10) and thus one may need to suppress both couplings in order to not enhance the single top production cross section. Given the complexity of the final states, a direct comparison with the $\mathrm{D} \emptyset$ measurement is difficult to make.

\subsection{Results}

In this section I present results on the forward-backward asymmetry in $t \bar{t}$ production mediated by Higgs bosons in the S-MSSM, introduced in Chapter 4, as well as the contributions to the total $t \bar{t}$ cross section. Due to the large number of parameters that are present in the model, and due to the fact that there exists a vast region of parameter space that can provide a solution to the little hierarchy problem, I present the results for various values of $v_{s}$. Furthermore, I illustrate results for the two limiting cases of $\mu_{s}$ which were discussed in Sections 4.2 and 4.3. I use $\tan \beta=2$ with a corresponding value of $\lambda=0.63$ and work in the Higgs

decoupling limit in order to maximize the tree-level contribution to the mass of the SM-like Higgs boson. The scale of new physics, $M$, where the operators in Equation (6.9) arise if fixed to $1 \mathrm{TeV}$. 
In the calculations I make use the CTEQ6L PDF set [103] using a factorization and renormalization scale of $m_{t} / 2$. For the strong coupling constant I take $\alpha_{s}(161.9 \mathrm{GeV}) \sim 0.1$, which is used to calculate the one-loop radiative correction to the Higgs masses. The calculations are performed using a top mass of 172.5 GeV and I use the CDF analysis of the $t \bar{t}$ production cross section which incorporates a combination of leptonic and hadronic channels using data with an integrated luminosity of $4.6 \mathrm{fb}^{-1}[110]$. They find:

$$
\sigma_{t \bar{t}}=7.50 \pm 0.48 \mathrm{pb},
$$

for $m_{t}=172.5 \mathrm{GeV}$. In addition, all of the constraints introduced in Section 6.3 are applied to search for viable scenarios consistent with experimental observations.

In the small $\mu_{s}$ limit the vacuum structure of the theory is significantly different from that of the MSSM. In particular, the appearance of light mostly singlet scalars can significantly enhance the $t \bar{t}$ production cross section. In Figure 6.3 I illustrate the results of the new physics contributions to the $t \bar{t}$ cross section as a function of the forward-backward asymmetry. The output parameters that arise from electroweak symmetry breaking are shown in Table 6.1. In the figure I show the experimental value for the cross section with a green one sigma band and the experimental value for the asymmetry with a cyan one sigma band. As can be seen from the figure, for all of the curves there is a region that falls within one standard deviation from both cross section and asymmetry. The black dotted line corresponds to a value of $v_{S}=120 \mathrm{GeV}, \mu_{s}=20 \mathrm{GeV}$ and $A_{\lambda}=190 \mathrm{GeV}$ as well as vanishing values of $\mu$ and $B_{\mu}$, labeled scenario $\mathrm{A}$ in Table 6.1. Scenario A is characterized by a heavy scalar and pseudoscalar with masses around $200 \mathrm{GeV}$, a SM-like Higgs with mass $124 \mathrm{GeV}$, one singlet-like scalar with mass $85 \mathrm{GeV}$ and 


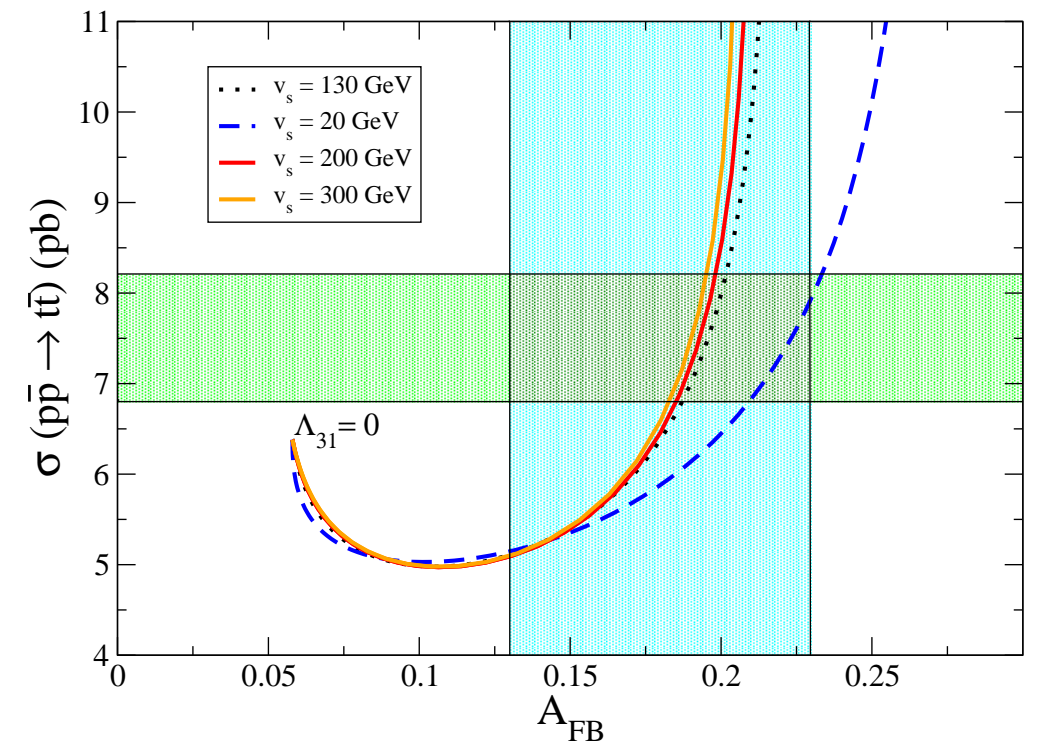

Figure 6.3. The $t \bar{t}$ production cross section as a function of the parton level forward-backward top asymmetry for various values of the singlet vev $v_{s}$, Scenarios A through D. The green band indicate the combined uncertainty from the asymmetry measurements of CDF and $\mathrm{D} \varnothing[2,3]$, and the cyan band the combined theoretical and experimental uncertainty on the value of the $t \bar{t}$ production cross section given in Equation (6.31) [110]. The value of $\Lambda_{31}$ increases along the curves, from 0 (left) to 9.5 (right) for $\Lambda_{13}$ close to zero.

one singlet-like pseudoscalar with a mass of $60 \mathrm{GeV}$. This mass splitting between the two singlet-like states, which is evident from Equations (4.35) and (4.34), is due to the fact that the ratio $A_{\lambda}^{2} / m_{A}^{2}$ approaches unity. The blue dotted line corresponds to a value of $v_{S}=20 \mathrm{GeV}, \mu_{s}=20 \mathrm{GeV}$ and $A_{\lambda}=470 \mathrm{GeV}$ as well as values for $\mu$ and $\sqrt{B_{\mu}}$ of 180 and $500 \mathrm{GeV}$ respectively, labeled scenario B in Table 6.1. Scenario B is characterized by a heavy scalar and pseudoscalar with masses around $800 \mathrm{GeV}$, a SM-like Higgs with mass $124 \mathrm{GeV}$, one singletlike scalar and pseudoscalar with masses close to $\sim 100 \mathrm{GeV}$. The near mass degeneracy of the singlet-like states is apparent from Equations (4.35) and (4.34) in Section 4.3, given that the $A_{\lambda}^{2} / m_{A}^{2}$ ratio has a more negligible contribution to 
the masses. In Figure $6.4 \mathrm{I}$ plot the asymmetry as a function of $\Lambda_{31}$ on the left, and the total cross section as function of $\Lambda_{31}$ on the right for scenarios $\mathrm{A}$ and $\mathrm{B}$. The value of $\Lambda_{13}$ is fixed close to zero in order to remain consistent mainly with the constraint arising from the up quark mass. In this figure, the impact that the lighter spectrum has on the cross section becomes more evident and they become more dominant in scenario $\mathrm{A}$ for smaller values of $\Lambda_{31}$. From Figures 6.3 and 6.4 one can also note the inflection point where the pure new physics contributions to the cross section dominate over the interference terms in Equations (6.19) and (6.20). This transition from negative to positive contributions to the cross sections is more rapid for smaller values of $\Lambda_{31}$ and larger values of $v_{s}$, and it is also a consequence of the relatively light spectrum. In scenarios C and D (red and orange in Figure 6.3, respectively) the value of $v_{s}$ is increased by increasing $A_{\lambda}$ to 310 and $470 \mathrm{GeV}$, respectively. The values of $\mu$ and $B_{\mu}$ are fixed to zero. The light Higgs spectrum for these two scenarios remains identical to that of scenario A, since the ratio of $A_{\lambda}^{2} / m_{A}^{2}$ remains close to unity. A large value of $v_{s}$ thus requires a smaller value of $\Lambda_{31}$ to generate a significant contribution to the cross section.

In the large $\mu_{s}$ limit, the singlet decouples from the theory and in the Higgs decoupling limit the only light scalar is the SM-like Higgs. Furthermore, within this class of models $v_{s} \rightarrow 0$ and the most dominant contribution to the cross section and asymmetry arises from the coupling of the SM-like Higgs to the up and top quarks which is proportional to

$$
\frac{\left(\Lambda_{13,31}\right) v \sin \beta}{M} O_{1, S}^{H}
$$



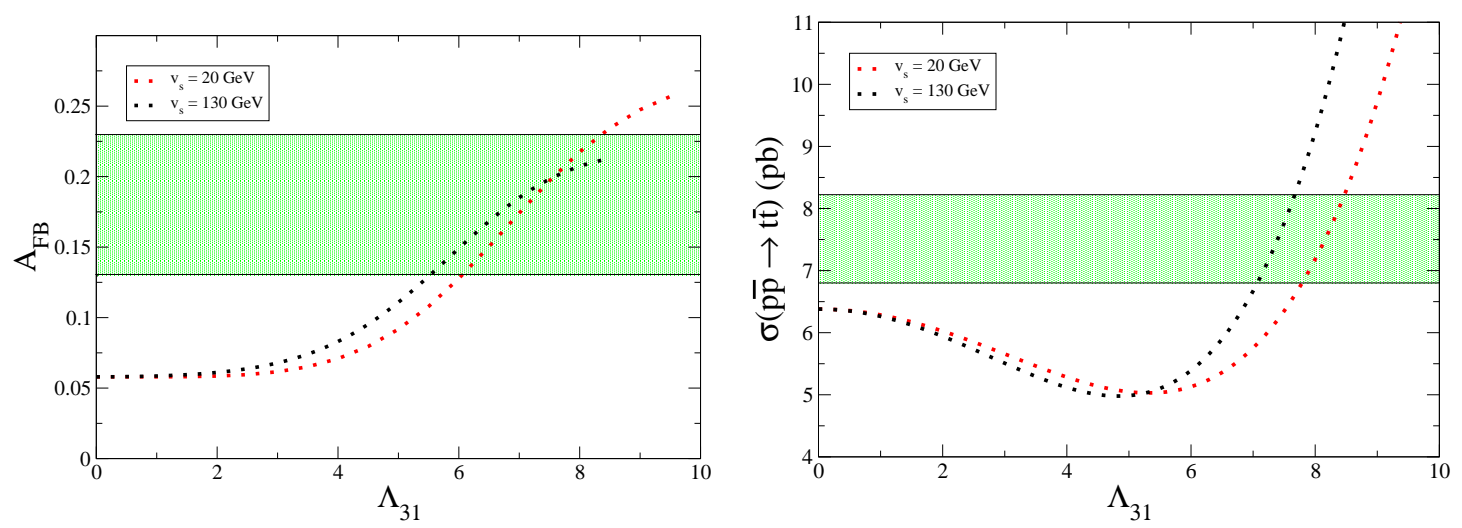

Figure 6.4. On the left plot the forward-backward top asymmetry at the parton level as a function of $\Lambda_{31}$ for scenarios $\mathrm{A}$ and $\mathrm{B}$. The green bands indicate the combined uncertainty from the asymmetry measurements of CDF and $\mathrm{D} \varnothing[2,3]$. On the right, the $t \bar{t}$ production cross section as a function of $\Lambda_{31}$ for scenarios A and B. The green bands indicate the combined theoretical and experimental uncertainty on the cross section [110].
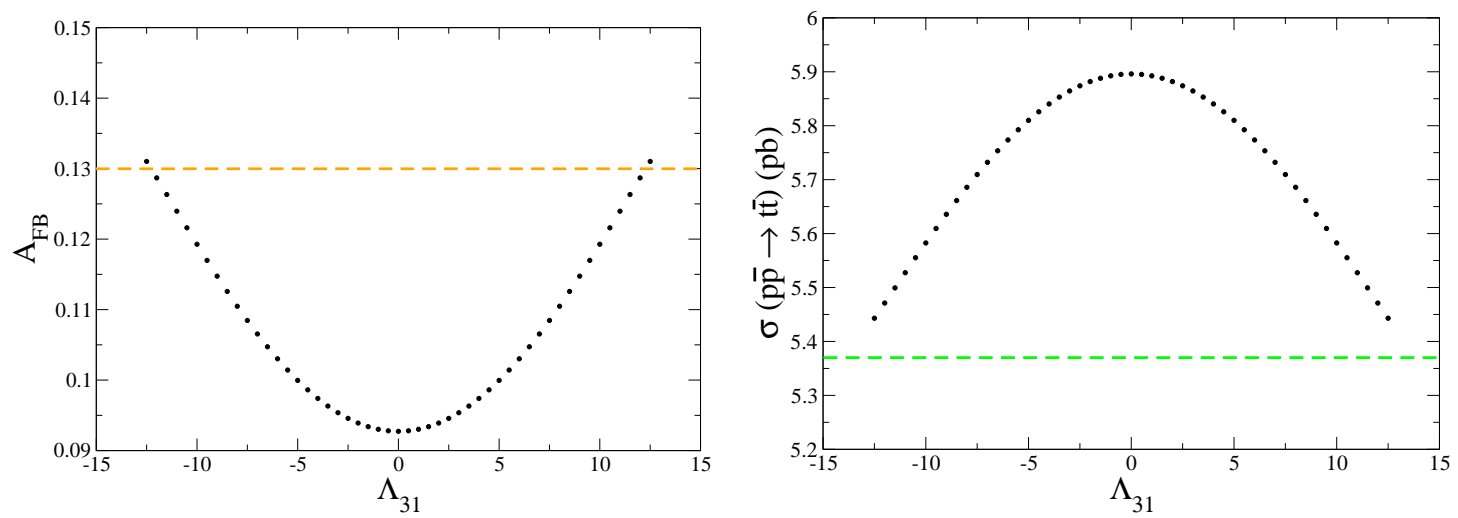

Figure 6.5. On the left plot the forward-backward top asymmetry at the parton level as a function of $\Lambda_{31}$ for the large $\mu_{s}$ scenario. The orange line indicates a one $\sigma$ deviation from a combination of the independent CDF and $\mathrm{D} \varnothing$ asymmetry measurements $[2,3]$. On the right, the $t \bar{t}$ production cross section as a function of $\Lambda_{31}$. The green line corresponds to three $\sigma$ deviations away from the experimental cross section [110]. 
TABLE 6.1

SCALAR MIXING ANGLES AND VEV IN THE SINGLET FIELD

DIRECTION.

\begin{tabular}{|c|c|c|c|c|}
\hline \hline & Sc. A & Sc. B & Sc. C & Sc. D \\
\hline \hline$v_{s}[\mathrm{GeV}]$ & 130 & 20 & 200 & 300 \\
$O_{2, S}^{H}, O_{2, H_{u}}^{H}$ & $-0.079,0.90$ & $0.024,-0.89$ & $-0.12,0.90$ & $-0.18,0.89$ \\
$O_{1, S}^{H}, O_{1, H_{u}}^{H}$ & $-0.091,0.93$ & $-0.0007,0.99$ & $0.01,0.97$ & $0.10,0.97$ \\
$O_{1, S}^{A}, O_{1, H_{u}}^{A}$ & -0.190 .90 & -0.030 .99 & $-0.13,0.95$ & $-0.095,0.98$ \\
\hline \hline
\end{tabular}

The value of $O_{1, S}^{H}$ is very small since the SM-like Higgs has a very little singlet component, hence the additional suppression. In the analysis, I fix the $\mu$ parameter to be consistent with searches of supersymmetric particles carried out by LEP [94]. The main results are shown in Figure 6.5. On the left I have plotted the total $t \bar{t}$ cross section and on the right the top forward-backward asymmetry as a function of the $\Lambda_{31}$ while fixing the value of $\Lambda_{13}=12.5$. For this figure I have chosen $\mu_{s}=1.5 \mathrm{TeV}, \mu=500 \mathrm{GeV}, A_{\lambda}=-1 \mathrm{TeV}$ and $B_{\mu}=(500 \mathrm{GeV})^{2}$ which yield a value of $v_{s}=0.5 \mathrm{GeV}$. It can be seen from the figure that even for rather large values of both $\Lambda_{13}$ and $\Lambda_{31}$, the interference contribution to the cross section always dominates. This is due to the additional suppression in the coupling of the SM-like Higgs to the up and top quarks, see Equation (6.32). Furthermore, an asymmetry above $13 \%$, that is within one sigma of the experimental result, can only be obtained when maximizing both $\Lambda_{13}$ and $\Lambda_{31}$. However, the corresponding cross section is close to being outside the three sigma region. Models with large $\mu_{s}$ and with only a relatively light scalar with SM-like couplings present a large amount of tension in the sense that in order to minimize the negative interference 
contributions to the cross section, one must sacrifice obtaining a large asymmetry. 


\section{CHAPTER 7}

\section{CONCLUSIONS AND OUTLOOK}

Supersymmetric extensions of the Standard Model have the property of avoiding the order-by-order fine tuning that one imposes on the Higgs mass parameter of the Standard Model; achieving this, by doubling the particle spectrum. However, for it to be a viable solution to the hierarchy problem, the new supersymmetric partners must lie close to the electroweak scale. Unfortunately, the minimal supersymmetric extension of the Standard Model has been pushed experimentally to regions of its parameter space where new tunings are required. In this thesis I examined an extension to the MSSM which can naturally evade experimental bounds without the necessity of new tunings.

In this work I incorporated additional matter coupling to the MSSM Higgs sector while also marrying it to explicit supersymmetric mass terms. The primary issue with this approach was sacrificing the ability to solve the $\mu$ problem of the MSSM. In return, I gained the ability to alleviate the little hierarchy problem in a more natural way, without the need of large radiative corrections to the Higgs mass parameter. In particular, I showed that the S-MSSM allows light Higgs masses above the LEP bound even with top squark masses well below the TeV scale, and does so without requiring any cancellations among the various parameters of the theory. I presented two different but extremely interesting limits of the SMSSM, characterized by the size of the supersymmetric mass term for the singlet 
superfield. I showed that both the large and small $\mu_{S}$ limits provide a natural solution to the little hierarchy problem. In addition, I pointed out that both limits would present themselves through different signatures at particle colliders. The large $\mu_{S}$ limit would manifest itself with a phenomenology very similar to that of the MSSM, except for a measured SM-like Higgs mass larger than the allowed by the observed top squark mass spectrum. In the large $\mu_{S}$ limit, the additional matter content consisting of a singlet-like scalar and pseudoscalar as well as a singlet-like fermion, would be to heavy to be probed with current particle collider energies, thus their presence would have to be felt indirectly through the mass of the SM-like Higgs boson. In the other hand, in the small $\mu_{S}$ limit, a number of new light scalars appear and these could have profound effects on the phenomenolgy at the LHC. I mentioned a particular signature due to the possibilty of scalar cascade decays where the SM-like Higgs boson would decay into a pair of singletlike scalars. These decay channels have no effect on current LHC searches, since at the LHC the main production channel for a SM-like Higgs boson is through gluon fusion and decays into a pair of photons. Instead, it would affect the $b \bar{b}$ decay channel which would turn out to be largely suppressed. Thus, in order to differentiate the S-MSSM from the Standard Model and the MSSM, the $b \bar{b}$ channel would have to be measured very precisely.

In this thesis, I discussed the two $\mu_{S}$ limits of the S-MSSM through a low energy framework, that is, the parameters used in the analysis were incorporated to solve the little hierarchy problem. Furthermore, I showed that a large area of parameter space had this property. However, there existed the possibility that these regions of parameter space could not be realized in a a realistic model of SUSY breaking, yielding a picture somewhat less natural than what it originally ap- 
peared to be. In this thesis I was able to embed the S-MSSM in the large $\mu_{S}$ limit into a gauge-mediated SUSY breaking scheme. Within this scheme, the number of free parameters was reduced significantly, yielding low-energy parameters that were highly constrained. From the results, I showed that the S-MSSM remained a viable solution to the little hierarchy problem once embedded into a minimal GMSB scenario, producing Higgs masses up to $122 \mathrm{GeV}$ without fine tuning in either the top squark sector or everywhere else in the model. Furthermore, the choices of parameters that gave Higgs masses above the LEP bound were quite generic. Within the context of GMSB, I showed in this work that the S-MSSM had an edge over the NMSSM, which required a non-minimal GMSB embedding. One major advantage of the S-MSSM is that it opened up parameter space that was previously unavailable in the NMSSM. In particular, the tunings that were required in the NMSSM in order to break the electroweak symmetry while producing a $\mu$-term large enough to pass the chagino mass contraints appeared to be absent here. By adding a singlet to the MSSM, but not requiring that it solve the $\mu$ problem, I found a phenomenologically viable, very minimal and quite interesting extension of the MSSM that naturally solved the little hierachy problem.

In this work, I also studied the forward-backward asymmetry in the $t \bar{t}$ system within the framework of the S-MSSM. Within this framework, I provided an explanation for the recent experimental observation of the asymmetric production of $t \bar{t}$ pairs by the CDF and $\mathrm{D} \varnothing$ collaborations. To tackle this problem, I incorporated dimension-five operators to the S-MSSM, coupling the first and third generation of quarks to scalars. I found that in the small $\mu_{S}$ limit I was able to generate asymmetries consistent with the CDF and D $\varnothing$ results while being consistent with other well founded experimental constraints. However, in the large $\mu_{S}$ limit, pro- 
ducing a large asymmetry was often in tension with the cross section due to large interference constributions arising from the dimension-five operators.

The LHC is narrowing down the window of masses where a SM-like Higgs boson mass may lie. In particular, the only regions left to experimentally exclude are those between 115 and $127 \mathrm{GeV}$. As mentioned at the end of Chapter 4, a SM-like Higgs boson discovered in this range would not only be a milestone for particle physics, but also signal that the discovery of supersymmetry could be the next major breakthrough. The S-MSSM, as a natural solution to the little hierarchy problem, is an outstanding candidate for a supersymmetric extension of the Standard Model but it does not attempt to explain the source of the supersymmetric mass terms. One primary assumption made in the analysis of the S-MSSM was that the eventual understanding of how $\mu$ and $\mu_{S}$ are generated would not disrupt the scalar potential at the electroweak scale. The idea of tackling the $\mu$-problem, and to an extent a supersymmetric mass problem, together with the little hierarchy problem within the same framework is very compelling, and one I wish to study in greater detail; it will definitly shape my future work in regards to supersymmetric extensions of the MSSM. Ultimately, the direction of my future research will be guided by what the LHC reveals about nature, but I want to emphasize that the S-MSSM remains a strong candiadate for physics beyond the Standard Model. 


\section{APPENDIX A}

In this Appendix I provide the renormalization group equations (RGEs) of the NMSSM in the DR scheme $[62,90]$. The RGEs are implemented at one-loop order for the soft masses and at two loops for the dimensioneless couplings. Since the S-MSSM does not incorporate any new dimensionless couplings, the relevant RGEs are those of the NMSSM with $\kappa=A_{\kappa}=0$. In this work, the RGEs for $\mu$ and $\mu_{S}$ play no role in the analysis of the S-MSSM and are not included.

\section{A.1 Gauge and Yukawa couplings}

In this section I include the RGE's for gauge and Yukawa couplings at twoloop order. The Yukawa couplings for the first and second families are largely suppressed relative to the thrid family and are omitted. Additionally, mixing among quark families parametrized by the CKM matrix is not taken into account due to their small effect on the equations.

$$
\begin{aligned}
16 \pi^{2} \frac{d g_{1}^{2}}{d t} & =11 g_{1}^{4}+\frac{g_{1}^{4}}{16 \pi^{2}}\left(\frac{199}{9} g_{1}^{2}+9 g_{2}^{2}+\frac{88}{3} g_{3}^{2}-\frac{26}{3} y_{t}^{2}-\frac{14}{3} y_{b}^{2}-6 y_{\tau}^{2}-2 \lambda^{2}\right) \\
16 \pi^{2} \frac{d g_{2}^{2}}{d t} & =g_{2}^{4}+\frac{g_{2}^{4}}{16 \pi^{2}}\left(3 g_{1}^{2}+25 g_{2}^{2}+24 g_{3}^{2}-6 y_{t}^{2}-6 y_{b}^{2}-2 y_{\tau}^{2}-2 \lambda^{2}\right) \\
16 \pi^{2} \frac{d g_{3}^{2}}{d t} & =-3 g_{3}^{4}+\frac{g_{3}^{4}}{16 \pi^{2}}\left(\frac{11}{3} g_{1}^{2}+9 g_{2}^{2}+14 g_{3}^{2}-4 y_{t}^{2}-4 y_{b}^{2}\right)
\end{aligned}
$$




$$
\begin{aligned}
16 \pi^{2} \frac{d y_{t}^{2}}{d t} & =y_{t}^{2}\left(6 y_{t}^{2}+y_{b}^{2}+\lambda^{2}-\frac{13}{9} g_{1}^{2}-3 g_{2}^{2}-\frac{16}{3} g_{3}^{2}\right) \\
& +\frac{y_{t}^{2}}{16 \pi^{2}}\left(-22 y_{t}^{4}-5 y_{b}^{4}-3 \lambda^{4}-5 y_{t}^{2} y_{b}^{2}-3 y_{t}^{2} \lambda^{2}-y_{b}^{2} y_{\tau}^{2}-4 y_{b}^{2} \lambda^{2}\right. \\
& -y_{\tau}^{2} \lambda^{2}-2 \lambda^{2} \kappa^{2}+2 g_{1}^{2} y_{t}^{2}+\frac{2}{3} g_{1}^{2} y_{b}^{2}+6 g_{2}^{2} y_{t}^{2}+16 g_{3}^{2} y_{t}^{2} \\
& \left.+\frac{2743}{162} g_{1}^{4}+\frac{15}{2} g_{2}^{4}-\frac{16}{9} g_{3}^{4}+\frac{5}{3} g_{1}^{2} g_{2}^{2}+\frac{136}{27} g_{1}^{2} g_{3}^{2}+8 g_{2}^{2} g_{3}^{2}\right), \\
16 \pi^{2} \frac{d y_{b}^{2}}{d t} & =y_{b}^{2}\left(6 y_{b}^{2}+y_{t}^{2}+y_{\tau}^{2}+\lambda^{2}-\frac{7}{9} g_{1}^{2}-3 g_{2}^{2}-\frac{16}{3} g_{3}^{2}\right) \\
& +\frac{y_{b}^{2}}{16 \pi^{2}}\left(-22 y_{b}^{4}-5 y_{t}^{4}-3 y_{\tau}^{4}-3 \lambda^{4}-5 y_{b}^{2} y_{t}^{2}-3 y_{b}^{2} y_{\tau}^{2}-3 y_{b}^{2} \lambda^{2}\right. \\
& -4 y_{t}^{2} \lambda^{2}-2 \lambda^{2} \kappa^{2}+\frac{2}{3} g_{1}^{2} y_{b}^{2}+\frac{4}{3} g_{1}^{2} y_{t}^{2}+2 g_{1}^{2} y_{\tau}^{2}+6 g_{2}^{2} y_{b}^{2}+16 g_{3}^{2} y_{b}^{2} \\
& \left.+\frac{1435}{162} g_{1}^{4}+\frac{15}{2} g_{2}^{4}-\frac{16}{9} g_{3}^{4}+\frac{5}{3} g_{1}^{2} g_{2}^{2}+\frac{40}{27} g_{1}^{2} g_{3}^{2}+8 g_{2}^{2} g_{3}^{2}\right), \\
16 \pi^{2} \frac{d y_{\tau}^{2}}{d t} & =y_{\tau}^{2}\left(4 y_{\tau}^{2}+3 y_{b}^{2}+\lambda^{2}-3 g_{1}^{2}-3 g_{2}^{2}\right) \\
& +\frac{y_{\tau}^{2}}{16 \pi^{2}}\left(-10 y_{\tau}^{4}-9 y_{b}^{4}-3 \lambda^{4}-9 y_{\tau}^{2} y_{b}^{2}-3 y_{\tau}^{2} \lambda^{2}-3 y_{t}^{2} y_{b}^{2}-3 y_{t}^{2} \lambda^{2}\right. \\
& \left.-2 \lambda^{2} \kappa^{2}+2 g_{1}^{2} y_{\tau}^{2}-\frac{2}{3} g_{1}^{2} y_{b}^{2}+6 g_{2}^{2} y_{\tau}^{2}+16 g_{3}^{2} y_{b}^{2}+\frac{75}{2} g_{1}^{4}+\frac{15}{2} g_{2}^{4}+3 g_{1}^{2} g_{2}^{2}\right),
\end{aligned}
$$

$$
\begin{aligned}
16 \pi^{2} \frac{d \lambda^{2}}{d t} & =\lambda^{2}\left(3 y_{t}^{2}+3 y_{b}^{2}+y_{\tau}^{2}+4 \lambda^{2}+2 \kappa^{2}-g_{1}^{2}-3 g_{2}^{2}\right) \\
& +\frac{\lambda^{2}}{16 \pi^{2}}\left(-10 \lambda^{4}-9 y_{t}^{4}-9 y_{b}^{4}-3 y_{\tau}^{4}-8 \kappa^{4}-9 \lambda^{2} y_{t}^{2}-9 \lambda^{2} y_{b}^{2}\right. \\
& -3 \lambda^{2} y_{\tau}^{2}-12 \lambda^{2} \kappa^{2}-6 y_{t}^{2} y_{b}^{2}+2 g_{1}^{2} \lambda^{2}+\frac{4}{3} g_{1}^{2} y_{t}^{2}-\frac{2}{3} g_{1}^{2} y_{b}^{2}+2 g_{1}^{2} y_{\tau}^{2} \\
& \left.+6 g_{2}^{2} \lambda^{2}+16 g_{3}^{2} y_{t}^{2}+16 g_{3}^{2} y_{b}^{2}+\frac{23}{2} g_{1}^{4}+\frac{15}{2} g_{2}^{4}+3 g_{1}^{2} g_{2}^{2}\right) \\
16 \pi^{2} \frac{d \kappa^{2}}{d t} & =\kappa^{2}\left(6 \lambda^{2}+6 \kappa^{2}\right)+\frac{\kappa^{2}}{16 \pi^{2}}\left(-24 \kappa^{4}-12 \lambda^{4}-24 \kappa^{2} \lambda^{2}\right. \\
& \left.-18 y_{t}^{2} \lambda^{2}-18 y_{b}^{2} \lambda^{2}-6 y_{\tau}^{2} \lambda^{2}+6 g_{1}^{2} \lambda^{2}+18 g_{2}^{2} \lambda^{2}\right)
\end{aligned}
$$

where $t=\log (Q / \mathrm{GeV})$. 


\section{A.2 Gaugino Masses}

In this section I include the RGEs for gaugino masses at one-loop order.

$$
\begin{aligned}
16 \pi^{2} \frac{d M_{1}}{d t} & =11 g_{1}^{2} M_{1} \\
16 \pi^{2} \frac{d M_{2}}{d t} & =g_{2}^{2} M_{2}, \\
16 \pi^{2} \frac{d M_{3}}{d t} & =-3 g_{3}^{2} M_{3} .
\end{aligned}
$$

\section{A.3 Trilinear couplings}

In this section I include the RGEs for the trilinear couplings for the third generation at one-loop order as well as the RGEs for $A_{\lambda}$ and $A_{\kappa}$.

$$
\begin{aligned}
16 \pi^{2} \frac{d A_{t}}{d t} & =6 y_{t}^{2} A_{t}+y_{b}^{2} A_{b}+\lambda^{2} A_{\lambda}+\frac{13}{9} g_{1}^{2} M_{1}+3 g_{2}^{2} M_{2}+\frac{16}{3} g_{3}^{2} M_{3} \\
16 \pi^{2} \frac{d A_{b}}{d t} & =6 y_{b}^{2} A_{b}+y_{t}^{2} A_{t}+y_{\tau}^{2} A_{\tau}+\lambda^{2} A_{\lambda}+\frac{7}{9} g_{1}^{2} M_{1}+3 g_{2}^{2} M_{2}+\frac{16}{3} g_{3}^{2} M_{3} \\
16 \pi^{2} \frac{d A_{\tau}}{d t} & =4 y_{\tau}^{2} A_{\tau}+3 y_{b}^{2} A_{b}+\lambda^{2} A_{\lambda}+3 g_{1}^{2} M_{1}+3 g_{2}^{2} M_{2} \\
16 \pi^{2} \frac{d A_{\lambda}}{d t} & =4 \lambda^{2} A_{\lambda}+3 y_{t}^{2} A_{t}+3 y_{b}^{2} A_{b}+y_{\tau}^{2} A_{\tau}+2 \kappa^{2} A_{\kappa}+g_{1}^{2} M_{1}+3 g_{2}^{2} M_{2} \\
16 \pi^{2} \frac{d A_{\kappa}}{d t} & =6 \kappa^{2} A_{\kappa}+6 \lambda^{2} A_{\lambda} .
\end{aligned}
$$

\section{A.4 Squark, slepton and Higss soft masses}

In this section I include the RGEs for slepton, squark and Higgs soft SUSY breaking masses at one-loop order. The following parameters are defined in order 
to simplify the RGE expressions [62]:

$$
\begin{aligned}
M_{t}^{2} & =m_{Q_{3}}^{2}+m_{\bar{u}_{3}}^{2}+m_{H_{u}}^{2}+A_{t}^{2}, \\
M_{b}^{2} & =m_{Q_{3}}^{2}+m_{\bar{d}_{3}}^{2}+m_{H_{d}}^{2}+A_{b}^{2}, \\
M_{\tau}^{2} & =m_{L_{3}}^{2}+m_{\bar{e}_{3}}^{2}+m_{H_{d}}^{2}+A_{\tau}^{2}, \\
M_{\lambda}^{2} & =m_{H_{u}}^{2}+m_{H_{d}}^{2}+m_{S}^{2}+A_{\lambda}^{2}, \\
M_{\kappa}^{2} & =3 m_{S}^{2}+A_{\kappa}^{2}, \\
\xi & =\operatorname{Tr}\left(\mathbf{M}_{\mathbf{Q}}^{2}-2 \mathbf{M}_{\overline{\mathbf{u}}}^{2}+\mathbf{M}_{\overline{\mathbf{d}}}^{2}-\mathbf{M}_{\mathbf{L}}^{\mathbf{2}}+\mathbf{M}_{\overline{\mathbf{e}}}^{2}\right)+m_{H_{u}}^{2}-m_{H_{d}}^{2} .
\end{aligned}
$$

The RGEs are given by:

$$
\begin{aligned}
16 \pi^{2} \frac{d m_{Q_{a}}^{2}}{d t} & =\delta_{a 3} y_{t}^{2} M_{t}^{2}+\delta_{a 3} y_{b}^{2} M_{b}^{2}-\frac{1}{9} g_{1}^{2} M_{1}^{2}-3 g_{2}^{2} M_{2}^{2}-\frac{16}{3} g_{3}^{2} M_{3}^{2}+\frac{1}{6} g_{1}^{2} \xi \\
16 \pi^{2} \frac{d m_{\bar{u}_{a}}^{2}}{d t} & =2 \delta_{a 3} y_{t}^{2} M_{t}^{2}-\frac{16}{9} g_{1}^{2} M_{1}^{2}-\frac{16}{3} g_{3}^{2} M_{3}^{2}-\frac{2}{3} g_{1}^{2} \xi \\
16 \pi^{2} \frac{d m_{\bar{d}_{a}}^{2}}{d t} & =2 \delta_{a 3} y_{b}^{2} M_{b}^{2}-\frac{4}{9} g_{1}^{2} M_{1}^{2}-\frac{16}{3} g_{3}^{2} M_{3}^{2}+\frac{1}{3} g_{1}^{2} \xi \\
16 \pi^{2} \frac{d m_{L_{a}}^{2}}{d t} & =\delta_{a 3} y_{\tau}^{2} M_{\tau}^{2}-g_{1}^{2} M_{1}^{2}-3 g_{2}^{2} M_{2}^{2}-\frac{1}{2} g_{1}^{2} \xi \\
16 \pi^{2} \frac{d m_{\bar{e}_{a}}^{2}}{d t} & =2 \delta_{a 3} y_{\tau}^{2} M_{\tau}^{2}-4 g_{1}^{2} M_{1}^{2}+g_{1}^{2} \xi \\
16 \pi^{2} \frac{d m_{H_{u}}^{2}}{d t} & =3 y_{t}^{2} M_{t}^{2}+\lambda^{2} M_{\lambda}^{2}-g_{1}^{2} M_{1}^{2}-3 g_{2}^{2} M_{2}^{2}+\frac{1}{2} g_{1}^{2} \xi \\
16 \pi^{2} \frac{d m_{H_{d}}^{2}}{d t} & =3 y_{b}^{2} M_{b}^{2}+y_{\tau}^{2} M_{\tau}^{2}+\lambda^{2} M_{\lambda}^{2}-g_{1}^{2} M_{1}^{2}-3 g_{2}^{2} M_{2}^{2}-\frac{1}{2} g_{1}^{2} \xi .
\end{aligned}
$$




\section{BIBLIOGRAPHY}

1. T. Aaltonen et al. Phys.Rev.Lett., 105:232003, 2010.

2. T. Aaltonen et al. Phys.Rev., D83:112003, 2011.

3. V. M. Abazov et al. Phys.Rev., D84:112005, 2011.

4. V. M. Abazov et al. Phys.Lett., B705:313-319, 2011.

5. J. Abdallah et al. Eur.Phys.J., C31:421-479, 2003.

6. J. Aguilar-Saavedra and M. Perez-Victoria. Phys.Lett., B701:93-100, 2011.

7. I. Aitchison. Supersymmetry in Particle Physics. An Elementary Introduction. 2007.

8. Alexander and Kusenko. Nuclear Physics B - Proceedings Supplements, 52 (12):67 - 69, 1997.

9. L. Alvarez-Gaume, M. Claudson, and M. B. Wise. Nucl.Phys., B207:96, 1982.

10. Apostolos and Pilaftsis. Physics Letters B, 435(12):88 - 100, 1998.

11. K. Babu, I. Gogoladze, and C. Kolda. hep-ph/0410085, 2004.

12. R. Barate et al. Phys.Lett., B565:61-75, 2003.

13. R. Barbieri and A. Strumia. Phys.Lett., B433:63-66, 1998.

14. R. Barbieri, L. J. Hall, A. Y. Papaioannou, D. Pappadopulo, and V. S. Rychkov. JHEP, 0803:005, 2008.

15. G. Barenboim and G. Panotopoulos. JHEP, 1108:027, 2011.

16. P. Batra, A. Delgado, D. E. Kaplan, and T. M. Tait. JHEP, 0402:043, 2004.

17. P. Batra, A. Delgado, D. E. Kaplan, and T. M. Tait. JHEP, 0406:032, 2004. 
18. V. Bednyakov and H. Klapdor-Kleingrothaus. hep-ph/9809271, 1998.

19. B. Bellazzini, C. Csaki, A. Delgado, and A. Weiler. Phys.Rev., D79:095003, 2009.

20. A. Birkedal, Z. Chacko, and Y. Nomura. Phys.Rev., D71:015006, 2005.

21. K. Blum, Y. Hochberg, and Y. Nir. JHEP, 1110:124, 2011.

22. A. Brignole, J. Ellis, G. Ridolfi, and F. Zwirner. Physics Letters B, 271(12): $123-132,1991$.

23. A. Brignole, J. Casas, J. Espinosa, and I. Navarro. Nucl.Phys., B666:105143, 2003.

24. Q.-H. Cao, D. McKeen, J. L. Rosner, G. Shaughnessy, and C. E. Wagner. Phys.Rev., D81:114004, 2010.

25. M. Carena, T. Han, G.-Y. Huang, and C. E. Wagner. JHEP, 0804:092, 2008.

26. M. Carena, K. Kong, E. Ponton, and J. Zurita. Phys.Rev., D81:015001, 2010.

27. M. S. Carena, M. Quiros, and C. Wagner. Nucl.Phys., B461:407-436, 1996.

28. J. Casas, J. Espinosa, and I. Hidalgo. JHEP, 0401:008, 2004.

29. Z. Chacko, Y. Nomura, and D. Tucker-Smith. Nucl.Phys., B725:207-250, 2005.

30. S. Chang, C. Kilic, and R. Mahbubani. Phys.Rev., D71:015003, 2005.

31. S. Chang, P. J. Fox, and N. Weiner. JHEP, 0608:068, 2006.

32. S. Chang, L. J. Hall, and N. Weiner. Phys.Rev., D75:035009, 2007.

33. P. H. Chankowski, J. R. Ellis, M. Olechowski, and S. Pokorski. Nucl.Phys., B544:39-63, 1999.

34. S. Chatrchyan et al. axXiv:1202.1488 [hep-ex], 2012.

35. Collaboration, ATLAS. arXiv:1202.1408 [hep-ex], 2012.

36. D. Das and U. Ellwanger. JHEP, 1009:085, 2010.

37. A. de la Puente. JHEP, 1202:016, 2012.

38. A. Delgado and T. M. Tait. JHEP, 0507:023, 2005.

39. A. Delgado, G. Giudice, and P. Slavich. Phys.Lett., B653:424-433, 2007. 
40. A. Delgado, C. Kolda, J. P. Olson, and A. de la Puente. Phys.Rev., D82: 035006, 2010.

41. A. Delgado, C. Kolda, J. P. Olson, and A. de la Puente. Phys.Rev.Lett., 105: 091802, 2010.

42. A. Delgado, C. Kolda, and A. de la Puente. arXiv:111.4008 [hep-ph], 2011.

43. J.-P. Derendinger and C. Savoy. Nuclear Physics B, 237(2):307 - 328, 1984.

44. R. Dermisek and J. F. Gunion. Phys.Rev.Lett., 95:041801, 2005.

45. R. Dermisek and J. F. Gunion. Phys.Rev., D73:111701, 2006.

46. R. Dermisek and J. F. Gunion. Phys.Rev., D77:015013, 2008.

47. S. Di Chiara and K. Hsieh. Phys.Rev., D78:055016, 2008.

48. S. Dimopoulos and D. W. Sutter. Nucl.Phys., B452:496-512, 1995.

49. S. Dimopoulos, S. Raby, and F. Wilczek. Physics Letters B, 112(2):133 136, 1982.

50. M. Dine and W. Fischler. Phys.Lett., B110:227, 1982.

51. M. Dine and A. E. Nelson. Phys.Rev., D48:1277-1287, 1993.

52. M. Dine, A. E. Nelson, and Y. Shirman. Phys.Rev., D51:1362-1370, 1995.

53. M. Dine, A. E. Nelson, Y. Nir, and Y. Shirman. Phys.Rev., D53:2658-2669, 1996.

54. M. Dine, N. Seiberg, and S. Thomas. Phys.Rev., D76:095004, 2007.

55. B. A. Dobrescu, G. L. Landsberg, and K. T. Matchev. Phys.Rev., D63: 075003, 2001.

56. J. Ellis, G. Ridolfi, and F. Zwirner. Physics Letters B, 262(4):477 - 484, 1991.

57. J. Ellis, G. Ridolfi, and F. Zwirner. Physics Letters B, 257(12):83 - 91, 1991.

58. U. Ellwanger. Nucl.Phys.Proc.Suppl., 200-202:113-119, 2010.

59. U. Ellwanger and C. Hugonie. hep-ph/9901309, 1998.

60. U. Ellwanger, M. Rausch de Traubenberg, and C. A. Savoy. Nucl.Phys., B492:21-50, 1997. 
61. U. Ellwanger, C.-C. Jean-Louis, and A. Teixeira. JHEP, 0805:044, 2008.

62. U. Ellwanger, C. Hugonie, and A. M. Teixeira. Phys.Rept., 496:1-77, 2010.

63. J. Espinosa and M. Quiros. Phys.Lett., B279:92-97, 1992.

64. J. Espinosa and M. Quiros. Phys.Lett., B302:51-58, 1993.

65. J. R. Espinosa and M. Quiros. Phys.Rev.Lett., 81:516-519, 1998.

66. G. R. Farrar and P. Fayet. Physics Letters B, 76(5):575 - 579, 1978.

67. P. Fayet and J. Iliopoulos. Physics Letters B, 51(5):461 - 464, 1974.

68. R. Franceschini and S. Gori. JHEP, 1105:084, 2011.

69. G. Giudice and R. Rattazzi. Phys.Rept., 322:419-499, 1999.

70. P. W. Graham, A. Ismail, S. Rajendran, and P. Saraswat. Phys.Rev., D81: 055016, 2010.

71. B. Gripaios and S. M. West. Phys.Rev., D74:075002, 2006.

72. M. Grisaru, W. Siegel, and M. Roek. Nuclear Physics B, 159(3):429 - 450, 1979.

73. J. Gunion, H. Haber, and M. Sher. Nuclear Physics B, 306(1):1 - 13, 1988.

74. J. F. Gunion. J.Phys.Conf.Ser., 259:012012, 2010.

75. R. Haag, J. T. opuszaski, and M. Sohnius. Nuclear Physics B, 88(2):257 $274,1975$.

76. H. E. Haber and R. Hempfling. Phys. Rev. Lett., 66:1815-1818, Apr 1991.

77. H. E. Haber, R. Hempfling, and A. H. Hoang. Z.Phys., C75:539-554, 1997.

78. T. Hahn, S. Heinemeyer, W. Hollik, H. Rzehak, and G. Weiglein. Comput.Phys.Commun., 180:1426-1427, 2009.

79. L. J. Hall and T. Watari. Phys.Rev., D70:115001, 2004.

80. R. Harnik, G. D. Kribs, D. T. Larson, and H. Murayama. Phys.Rev., D70: 015002, 2004.

81. H.P. and Nilles. Physics Reports, 110(12):1 - 162, 1984.

82. C. Hugonie, G. Belanger, and A. Pukhov. JCAP, 0711:009, 2007. 
83. G. L. Kane and S. King. Phys.Lett., B451:113-122, 1999.

84. G. L. Kane, C. F. Kolda, and J. D. Wells. Phys.Rev.Lett., 70:2686-2689, 1993.

85. R. Kappl, M. Ratz, and M. W. Winkler. Phys.Lett., B695:169-173, 2011.

86. R. Kitano and Y. Nomura. Phys.Lett., B631:58-67, 2005.

87. L. and O'Raifeartaigh. Nuclear Physics B, 96(2):331 - 352, 1975.

88. A. Maloney, A. Pierce, and J. G. Wacker. JHEP, 0606:034, 2006.

89. S. P. Martin. hep-ph/9709356, 1997.

90. S. P. Martin and M. T. Vaughn. Phys.Rev., D50:2282, 1994.

91. D. Miller and R. Nevzorov. hep-ph/0309143, 2003.

92. T. Moroi and Y. Okada. Mod.Phys.Lett., A7:187-200, 1992.

93. T. Moroi and Y. Okada. Phys.Lett., B295:73-78, 1992.

94. K. Nakamura and P. D. Group. Journal of Physics G: Nuclear and Particle Physics, 37(7A):075021, 2010.

95. C. R. Nappi and B. A. Ovrut. Phys.Lett., B113:175, 1982.

96. G. Panotopoulos. arXiv:1103.0140 [hep-ph], 2011.

97. R. Peccei and H. R. Quinn. Phys.Rev.Lett., 38:1440-1443, 1977.

98. R. Peccei and H. R. Quinn. Phys.Rev., D16:1791-1797, 1977.

99. M. E. Peskin. arXiv:0801.1928 [hep-ph], pages 609-704, 2008.

100. M. E. Peskin and D. V. Schroeder. An Introduction To Quantum Field Theory (Frontiers in Physics). Westview Press, 1995.

101. Pierre and Fayet. Nuclear Physics B, 90(0):104 - 124, 1975.

102. A. Pilaftsis and C. E. Wagner. Nucl.Phys., B553:3-42, 1999.

103. J. Pumplin, D. Stump, J. Huston, H. Lai, P. M. Nadolsky, et al. JHEP, 0207:012, 2002.

104. M. Quiros. hep-ph/9901312, pages 187-259, 1999.

105. T. S. Roy and M. Schmaltz. JHEP, 0601:149, 2006. 
106. N. Sakai and T. Yanagida. Nuclear Physics B, 197(3):533 - 542, 1982.

107. P. C. Schuster and N. Toro. hep-ph/0512189, 2005.

108. O. Stal and G. Weiglein. JHEP, 1201:071, 2012.

109. The CDF and D0 Collaborations. arXiv:1107.5518 [hep-ex], 2011.

110. The CDF Collaboration. CDF Note 9913, 2009.

111. K. Tobe and J. D. Wells. Phys.Rev., D66:013010, 2002.

112. S. Weinberg. Phys. Rev. D, 26:287-302, Jul 1982.

113. J. Wess and J. Bagger. Supersymmetry and supergravity. 1992.

114. J. Wess and B. Zumino. Nuclear Physics B, 70(1):39 - 50, 1974.

This document was prepared 8 typeset with $\mathrm{L}_{\mathrm{E} X} 2_{\varepsilon}$, and formatted with NDdiss 2 classfile (v3.0[2005/07/27]) provided by Sameer Vijay. 